\title{
Fixed- $b$ Asymptotic Approximation of the Sampling Behavior of Nonparametric Spectral Density Estimators
}

\author{
Nigar Hashimzade \\ Department of Economics, University of Exeter \\ and \\ Timothy J. Vogelsang \\ Department of Economics, Michigan State University*
}

February 10, 2007

\begin{abstract}
We propose a new asymptotic approximation for the sampling behavior of nonparametric estimators of the spectral density of a covariance stationary time series. According to the standard approach, the truncation lag grows more slowly than the sample size. We derive first order limiting distributions under the alternative assumption that the truncation lag is a fixed proportion of the sample size. Our results extend the approach of Neave (1970) who derived a formula for the asymptotic variance of spectral density estimators under the same truncation lag assumption. We show that the limiting distribution of zero frequency spectral density estimators depends on how the mean is estimated and removed. The implications of our zero frequency results are consistent with exact results for bias and variance computed by $\mathrm{Ng}$ and Perron (1996). Finite sample simulations indicate that the new asymptotics provides a better approximation than the standard asymptotics.
\end{abstract}

Keywords: Zero frequency, bandwidth, kernel, truncation lag, inference.

${ }^{*}$ We thank participants at the 2003 Summer Meetings of the Econometric Society for helpful comments and suggestions. We thank Kin-Yip Ho for carefully proofreading an earlier version of the paper. Vogelsang gratefully acknowledges financial support from the National Science Foundation through grant SES-0525707. Corresponding Author: Tim Vogelsang, Department of Economics, 110 Marshall-Adams Hall, Michigan State University, East Lansing, MI 48824-1038, Phone: 517-432-1068, Fax: 517-432-1068, email: tjv@msu.edu 


\section{Introduction}

Spectral density estimation is an important and well established area of time series analysis. Much of the original work done on this topic was published in seminal papers going back over 50 years. Priestley (1981) provides an excellent review and discussion. This paper adds to the now very large literature on nonparametric spectral density by extending ideas first proposed by Neave (1970). A well-known class of nonparametric spectral density estimators takes the form of weighted sums of sample autocovariances. Asymptotic theory for these estimators has been developed under the assumption that a truncation lag parameter, $M$ (sometimes

called a bandwidth) increases at a rate slower than the sample size, T. Asymptotic normality of nonparametric spectral density estimators has been established under this assumption (see Grenander and Rosenblatt (1953)). In practice, a specific truncation lag must be chosen and the value of $b=M / T$ is positive. Although this asymptotic theory requires that $b$ go to zero as $T$ increases, in practice $b$ is greater than zero and can be nontrivially different from zero. Therefore, the traditional asymptotic theory can be labeled as "small- $b$ " asymptotics. Neave (1970) argued that a more accurate asymptotic variance formula could be developed by treating $b$ as a fixed constant as $T$ increases, thus mimicking the fact that $b$ is not zero in small samples. Neave (1970) derived asymptotic variance formulae based on this "fixed- $b$ " assumption.

In this paper we adopt the assumption that $b$ is a fixed constant as $T$ increases. We generalize the results of Neave (1970) and derive asymptotic distributions of nonparametric spectral density estimators. The distributions we obtain are nonstandard but can be expressed as functionals of standard Wiener processes. One interesting result we find is that asymptotic distributions of zero-frequency spectral density estimators depend on whether the data has been mean-corrected or detrended. This contrasts with the standard asymptotics where mean correction or detrending has no effect on the first order asymptotics at frequency zero. Our zero frequency results are qualitatively similar to exact results for bias and variance computed by Ng and Perron (1996). Because the fixed- $b$ asymptotic approximation captures much of the bias in zero frequency estimators when $b$ is not close to zero, it is a particularly useful result for serial correlation robust tests that use zero frequency spectral density estimators as standard errors. Capturing the bias reduces the tendency of 
serial correlation robust tests to over-reject when serial correlation in the errors is strong; see Kiefer and Vogelsang (2005).

The paper is organized as follows. In the next section the model is given and estimators are defined. Section 3 reviews well known asymptotic results under the standard small- $b$ approach. Section 4 presents the new fixed- $b$ asymptotic results. This section contains the theoretical contributions of the paper. Inference regarding the spectral density is briefly discussed in Section 5, and Section 6 presents a selection of Monte Carlo simulations that compare the accuracy of the small- $b$ and the fixed- $b$ asymptotics. All proofs are given in the appendix.

\section{The Model and Estimators}

Consider the time series process

$$
y_{t}=d_{t}+u_{t}, \quad t=1,2, \ldots, T
$$

where $u_{t}$ is a mean-zero covariance stationary time series process with autocovariance function

$$
\gamma_{j}=\operatorname{cov}\left(u_{t}, u_{t-j}\right)
$$

The component $d_{t}$ represents the deterministic part of $y_{t}$. Important examples include $d_{t}=0$ (a mean-zero time series process), $d_{t}=\mu$ (a time series process with mean $\mu$ ) and $d_{t}=\mu+\beta t$ (a trending time series process). Let $\omega \in[0, \pi]$ denote frequencies and define the spectral density of $u_{t}$ as

$$
f(\omega)=\frac{1}{2 \pi}\left[\gamma_{0}+\sum_{j=-\infty}^{\infty} \gamma_{j} \cos (\omega j)\right] .
$$

It is assumed that $0<f(\omega)<\infty$.

A well known class of estimators of $f(\omega)$ is the nonparametric class which takes the form

$$
\widehat{f}(\omega)=\frac{1}{2 \pi}\left[\widehat{\gamma}_{0}+\sum_{j=-(T-1)}^{T-1} k\left(\frac{j}{M}\right) \widehat{\gamma}_{j} \cos (\omega j)\right]
$$


where

$$
\begin{aligned}
& \widehat{\gamma}_{j}=T^{-1} \sum_{t=j+1}^{T} \widehat{u}_{t} \widehat{u}_{t-j}, \\
& \widehat{u}_{t}=y_{t}-\widehat{d}_{t},
\end{aligned}
$$

and $\widehat{d}_{t}$ is an estimate of $d_{t}$. Estimators of the form given by (2) were first proposed (in a slightly more general form) in the time series literature by Grenander and Rosenblatt (1953). The function $k(x)$ is a weighting function, or kernel, that typically downweights high lag sample autocovariances. $k(x)$ is an even function with $k(0)=1$ and $k(x)$ declines to zero as $x \rightarrow \infty$. Well known kernels have been proposed by Bartlett (1950), Daniell (1946), Parzen (1961) and Tukey (1949), among many others. See Priestley (1981) for a general discussion. The truncation lag, $M$, controls the amount of downweighting that is applied to the high order sample autocovariances. Consistency of $\widehat{f}(\omega)$ requires that $M \rightarrow \infty$ and $M / T \rightarrow 0$ as $T \rightarrow \infty$.

\section{Asymptotic Normality}

It has been shown that, under suitable regularity conditions, $\widehat{f}(\omega)$ has an asymptotically normal distribution. Sufficient regularity conditions for obtaining such a result are that $d_{t}$ is a linear polynomial in $t, u_{t}=\sum_{j=0}^{\infty} \psi_{j} \varepsilon_{t-j}$ where $\left\{\varepsilon_{t}\right\}$ is an i.i.d. process with $E\left(\varepsilon_{t}\right)=0$, $E\left[\varepsilon_{t}^{2}\right]<\infty, E\left[\varepsilon_{t}^{4}\right]<\infty$ and $\sum_{j=0}^{\infty}\left|\psi_{j}\right|<\infty$ (see Anderson (1971)). Under these conditions it follows that

$$
\sqrt{\frac{T}{M}}(\widehat{f}(\omega)-f(\omega)) \rightarrow^{d} N(0, V(\omega)),
$$

where

$$
V(\omega)=\left\{\begin{array}{ccc}
2 f^{2}(\omega) \int_{-\infty}^{\infty} k^{2}(x) d x & \text { for } & \omega=0, \pi \\
f^{2}(\omega) \int_{-\infty}^{\infty} k^{2}(x) d x & \text { for } & 0<\omega<\pi
\end{array}\right.
$$

\section{Fixed-b Asymptotic Approximation}

In this section an alternative asymptotic approximation for the sampling behavior of $\widehat{f}(\omega)$ is developed. The approach taken here is in the spirit of Neave (1970) who argued that while the assumption that $b=M / T \rightarrow 0$ is convenient mathematically and ensures consistency of $\widehat{f}(\omega)$, a more accurate approximation for the sampling variance of $\widehat{f}(\omega)$ can be obtained 
under the assumption that $M=[b T]$ where $b \in(0,1]$. Under this alternative assumption for $M$, Neave (1970) proved that

$$
\lim _{T \rightarrow \infty} \frac{T}{M} \operatorname{var}(\widehat{f}(\omega))=\left\{\begin{array}{cl}
2 f^{2}(\omega) \int_{-1 / b}^{1 / b} k^{2}(x)(1-b|x|) d x & \text { for } \quad \omega=0, \pi \\
f^{2}(\omega) \int_{-1 / b}^{1 / b} k^{2}(x)(1-b|x|) d x & \text { for } \quad 0<\omega<\pi
\end{array}\right.
$$

and these expressions become the standard formulae for $b=0$. It is important to note that this result by Neave (1970) does not apply to mean-corrected or detrended data for $\omega=0$. Part of Neave's proof follows a proof by Parzen (1957) and there appears to be a mistake (or typesetting error) on page 340 of Parzen (1957) that does not affect Parzen's proof but affects Neave's proof. Specifically, to show that mean-correcting or detrending have no asymptotic effects on $\widehat{f}(0)$, Parzen (1957) requires a term involving the scaled integral of the kernel, $k(x)$, to be $o(1)$. See the third equation on page 340 of Parzen (1957). Parzen argues that this term is bounded from above by a constant times $T^{-\varepsilon}$ where $\varepsilon>0$ is a fixed constant. The error is that the bound should be proportional to $(T / M)^{-\varepsilon}$ not $T^{-\varepsilon}$. Obviously, under the standard small- $b$ asymptotics, $T / M \rightarrow \infty$ as $T \rightarrow \infty$ in which case $(T / M)^{-\varepsilon}=o(1)$ and Parzen's proof goes through as argued. The problem for Neave's proof is the claim on page 72 of Neave (1970) that formulae given by his equation (2.5) follow from arguments in Parzen (1957) and do not depend on the condition that $M / T \rightarrow 0$. This claim is true for $\omega \neq 0$, whereas for $\omega=0$ it is only true if the data is know to be mean zero $\left(d_{t}=0\right)$. It does not hold for $\omega=0$ if the data is mean-corrected or detrended.

The appeal of Neave's approach is that it provides an approximation that reflects the fact that $M / T>0$ in finite samples. The limitation of Neave's result is that it only provides a formula for the variance and it does not address bias of $\widehat{f}(\omega)$ or indeed the distribution of $\widehat{f}(\omega)$. Building on the approach of Kiefer and Vogelsang (2005) who focused only on the case of $\omega=0$ for mean-corrected data, it is possible to extend Neave's result to the entire distribution of $\widehat{f}(\omega)$.

A first order asymptotic distribution theory for $\widehat{f}(\omega)$ can be developed under fairly general regularity conditions. Define the partial sum processes

$$
S_{t}^{c}(\omega)=\sum_{j=1}^{t} \cos (\omega j) u_{j}, \quad S_{t}^{s}(\omega)=\sum_{j=1}^{t} \sin (\omega j) u_{j}
$$

The key requirement for our results is that the following functional central limit theorems 
hold for these partial sums:

$$
\begin{gathered}
T^{-1 / 2} S_{[r T]}^{c}(0)=T^{-1 / 2} \sum_{t=1}^{[r T]} u_{t} \Rightarrow \sqrt{2 \pi f(0)} W(r), \\
T^{-1 / 2} S_{[r T]}^{c}(\pi)=T^{-1 / 2} \sum_{t=1}^{[r T]}(-1)^{t} u_{t} \Rightarrow \sqrt{2 \pi f(\pi)} W^{*}(r), \\
T^{-1 / 2} S_{[r T]}^{c}(\omega) \Rightarrow \sqrt{\pi f(\omega)} W_{1}(r), \quad 0<\omega<\pi, \\
T^{-1 / 2} S_{[r T]}^{s}(\omega) \Rightarrow \sqrt{\pi f(\omega)} W_{2}(r), \quad 0<\omega<\pi,
\end{gathered}
$$

where $W(r), W^{*}(r), W_{1}(r)$ and $W_{2}(r)$ are standard independent Wiener processes, $[r T]$ is the integer part of $r T$ with $r \in(0,1]$ and $\Rightarrow$ denotes weak convergence. Note that it trivially follows that $T^{-1 / 2} S_{[r T]}^{s}(0)=T^{-1 / 2} S_{[r T]}^{s}(\pi)=0$ because $\sin (0)=\sin (\pi t)=0$. A sufficient condition under which (4) - (7) hold is that $u_{t}=\sum_{j=0}^{\infty} \psi_{j} \varepsilon_{t-j}$ where $\left\{\varepsilon_{t}\right\}$ is i.i.d. with $E\left(\varepsilon_{t}\right)=0, E\left[\varepsilon_{t}^{2}\right]<\infty, E\left[\varepsilon_{t}^{\kappa}\right]$ for some $\kappa \geq 4$ and $\sum_{j=0}^{\infty} \psi_{j}^{2}<\infty$. See Theorems 1 and 2 of Boutahar (2006). These regularity conditions are similar to the regularity conditions used in the standard approach, see Priestley (1981, p.469).

The asymptotic distribution of $\widehat{f}(0)$ depends on $\widehat{d}_{t}$. We consider three cases for $d_{t}$ and $\widehat{d}_{t}$. Case (i): $d_{t}=0, \widehat{d}_{t}=0$. Case (ii): $d_{t}=\mu, \widehat{d}_{t}=\bar{y}=T^{-1} \sum_{t=1}^{T} y_{t}$. Case (iii): $d_{t}=\mu+\beta t$, $\widehat{d}_{t}=\widehat{\mu}+\widehat{\beta} t$ where $\widehat{\mu}$ and $\widehat{\beta}$ are the least squares estimates from a regression of $y_{t}$ on $(1, t)$. The limiting distribution of $\widehat{f}(0)$ can be expressed in terms of mean-corrected and detrended $W(r)$ which we denote by $\widehat{W}(r)$ and is defined as follows. For case (i) $\widehat{W}(r)=W(r)$. For cases (ii) and (iii)

$$
\widehat{W}(r)=W(r)-\left(\int_{0}^{r} g(s) d s\right)\left(\int_{0}^{1} g(s) g(s)^{\prime} d s\right)^{-1} \int_{0}^{1} g(s) d W(s),
$$

where $g(s)=1$ for case (ii) and $g(s)=[1, s]^{\prime}$ for case (iii). Note that $\widehat{W}(1)=0$ for cases (ii) and (iii).

Define

$$
k_{-}^{\prime}(1)=\lim _{h \rightarrow 0}[(k(1)-k(1-h)) / h],
$$

i.e. $k_{-}^{\prime}(1)$ is the derivative of $k(x)$ from the left at $x=1$. The following theorem provides the main results of the paper. The proof is given in the appendix. 
Theorem 1 Let $y_{t}$ be given by (1) where $u_{t}$ is a mean zero stationary process that satisfies (4) - (7) and suppose that $0<f(\omega)<\infty$. Let $M=b T$ with $b \in(0,1]$. The following hold as $T \rightarrow \infty$ :

1. If $k^{\prime \prime}(x)$ exists and is continuous, then for $0<\omega<\pi$ :

$$
\begin{aligned}
\frac{\widehat{f}(\omega)}{f(\omega)} \Rightarrow \frac{1}{2} \sum_{i=1}^{2}\left[-\frac{1}{b^{2}} \int_{0}^{1} \int_{0}^{1}\right. & \left.k^{\prime \prime}\left(\frac{r-s}{b}\right) W_{i}(r) W_{i}(s) d r d s\right] \\
& +\frac{1}{2} \sum_{i=1}^{2}\left[\frac{2}{b} W_{i}(1) \int_{0}^{1} k^{\prime}\left(\frac{1-r}{b}\right) W_{i}(r) d r+W_{i}(1)^{2}\right],
\end{aligned}
$$

for $\omega=\pi$ :

$\frac{\widehat{f}(\pi)}{f(\pi)} \Rightarrow-\frac{1}{b^{2}} \int_{0}^{1} \int_{0}^{1} k^{\prime \prime}\left(\frac{r-s}{b}\right) W^{*}(r) W^{*}(s) d r d s+\frac{2}{b} W^{*}(1) \int_{0}^{1} k^{\prime}\left(\frac{1-r}{b}\right) W^{*}(r) d r+W^{*}(1)^{2}$,

for $\omega=0$ :

$\frac{\widehat{f}(0)}{f(0)} \Rightarrow-\frac{1}{b^{2}} \int_{0}^{1} \int_{0}^{1} k^{\prime \prime}\left(\frac{r-s}{b}\right) \widehat{W}(r) \widehat{W}(s) d r d s+\frac{2}{b} \widehat{W}(1) \int_{0}^{1} k^{\prime}\left(\frac{1-r}{b}\right) \widehat{W}(r) d r+\widehat{W}(1)^{2}$.

2. If $k(x)$ is continuous, $k(x)=0$ for $|x| \geq 1$, and $k(x)$ is twice continuously differentiable everywhere except for possibly $|x|=1$, then for $0<\omega<\pi$ :

$$
\begin{array}{r}
\frac{\widehat{f}(\omega)}{f(\omega)} \Rightarrow \frac{1}{2} \sum_{i=1}^{2}\left[-\frac{1}{b^{2}} \iint_{|r-s| \leq b} k^{\prime \prime}\left(\frac{r-s}{b}\right) W_{i}(r) W_{i}(s) d r d s+\frac{2}{b} k_{-}^{\prime}(1) \int_{0}^{1-b} W_{i}(r) W_{i}(r+b) d r\right] \\
+\frac{1}{2} \sum_{i=1}^{2}\left[\frac{2}{b} W_{i}(1) \int_{1-b}^{1} k^{\prime}\left(\frac{1-r}{b}\right) W_{i}(r) d r+W_{i}(1)^{2}\right],
\end{array}
$$

for $\omega=\pi$ :

$$
\begin{aligned}
\frac{\widehat{f}(\pi)}{f(\pi)} \Rightarrow-\frac{1}{b^{2}} \iint_{|r-s| \leq b} k^{\prime \prime}\left(\frac{r-s}{b}\right) & W^{*}(r) W^{*}(s) d r d s+\frac{2}{b} k_{-}^{\prime}(1) \int_{0}^{1-b} W^{*}(r) W^{*}(r+b) d r \\
& +\frac{2}{b} W^{*}(1) \int_{1-b}^{1} k^{\prime}\left(\frac{1-r}{b}\right) W^{*}(r) d r+W^{*}(1)^{2}
\end{aligned}
$$

and for $\omega=0$ :

$$
\begin{aligned}
\frac{\widehat{f}(0)}{f(0)} \Rightarrow-\frac{1}{b^{2}} \iint_{|r-s| \leq b} k^{\prime \prime}\left(\frac{r-s}{b}\right) & \widehat{W}(r) \widehat{W}(s) d r d s+\frac{2}{b} k_{-}^{\prime}(1) \int_{0}^{1-b} \widehat{W}(r) \widehat{W}(r+b) d r \\
& +\frac{2}{b} \widehat{W}(1) \int_{1-b}^{1} k^{\prime}\left(\frac{1-r}{b}\right) \widehat{W}(r) d r+\widehat{W}(1)^{2}
\end{aligned}
$$


3. If $k(x)=1-|x|$ for $|x| \leq 1$ and $k(x)=0$ otherwise, i.e. $k(x)$ is the Bartlett kernel, then for $0<\omega<\pi$ :

$$
\begin{aligned}
& \frac{\widehat{f}(\omega)}{f(\omega)} \Rightarrow \frac{1}{2} \sum_{i=1}^{2}\left[\frac{2}{b} \int_{0}^{1} W_{i}(r)^{2} d r-\frac{2}{b} \int_{0}^{1-b} W_{i}(r) W_{i}(r+b) d r-\frac{2}{b} W_{i}(1) \int_{1-b}^{1} W_{i}(r) d r+W_{i}(1)^{2}\right], \\
& \text { for } \omega=\pi: \\
& \frac{\widehat{f}(\pi)}{f(\pi)} \Rightarrow \frac{2}{b} \int_{0}^{1} W^{*}(r)^{2} d r-\frac{2}{b} \int_{0}^{1-b} W^{*}(r) W^{*}(r+b) d r-\frac{2}{b} W^{*}(1) \int_{1-b}^{1} W^{*}(r) d r+W^{*}(1)^{2},
\end{aligned}
$$

and for $\omega=0$ :

$$
\frac{\widehat{f}(0)}{f(0)} \Rightarrow \frac{2}{b} \int_{0}^{1} \widehat{W}(r)^{2} d r-\frac{2}{b} \int_{0}^{1-b} \widehat{W}(r) \widehat{W}(r+b) d r-\frac{2}{b} \widehat{W}(1) \int_{1-b}^{1} \widehat{W}(r) d r+\widehat{W}(1)^{2}
$$

The theorem shows that limiting distribution of $\widehat{f}(\omega)$ is proportional to $f(\omega)$ under the assumption that $M=b T$. Similar to the standard asymptotics, different limits are obtained for $\omega=0$ compared to $\omega \neq 0$. Unlike in the standard approach, the limits in the $\omega=0$ case depend on the estimated deterministic trend, $\widehat{d}_{t}$.

The limiting distribution theory for $\omega=0$ was used by Kiefer and Vogelsang (2005) for mean-corrected data and by Bunzel and Vogelsang (2005) for detrended data to obtain a more accurate asymptotic theory for tests that use $\widehat{f}(0)$ when constructing standard errors. When $\widehat{f}(0)$ is used to estimate an asymptotic variance, consistency of $\widehat{f}(0)$ is usually the approximation used when determining how the sampling behavior of $\widehat{f}(0)$ affects the ultimate test. Thus, $\widehat{f}(0)$ is approximated by $f(0)$. Because $\widehat{f}(0)$ can exhibit severe downward bias, using the random variables in Theorem 1 to approximate $\widehat{f}(0)$ yields a more accurate asymptotic approximation since some of the bias and sampling variability in $\widehat{f}(0)$ is captured by the fixed- $b$ asymptotics. A formal result along these lines has been established by Phillips, Sun and Jin (2005)a for the case where $d_{t}=1$ and $u_{t}$ is Gaussian.

A recent paper by Phillips, Sun and Jin (2005)b develops an asymptotic theory analogous to the results in Theorem 1 for the case of exponentiated kernels. Specifically, Phillips et al. (2005)b consider estimators of $f(\omega)$ where in $(2) k\left(\frac{j}{M}\right)$ is replaced with $k\left(\frac{j}{T}\right)^{\gamma}$ where $\gamma$ is a positive real number. Increasing $\gamma$ places less weight on higher-order sample autocovariances in much the same way as does smaller values of $M$. Holding $\gamma$ fixed as $T$ increases leads 
to an asymptotic theory that is analogous to fixed- $b$ asymptotics. In addition to providing fixed- $\gamma$ asymptotic results, Phillips et al. (2005)b also derive a more traditional asymptotic normal approximation under the assumption that $\gamma$ increases with $T$ but at a slower rate.

\section{Finite Sample Comparison of the Normal and Fixed- $b$ Asymptotic Approxi- mations}

One standard metric by which to judge an asymptotic approximation is how well the asymptotic distribution approximates the sampling distribution of the statistic. Suppose for a given sample of size $T$, a particular value of $M$ is used to construct $\widehat{f}(\omega)$. The question is then which asymptotic approximation, the normal approximation based on standard small- $b$ asymptotics or the new fixed- $b$ asymptotics, is more accurate.

We performed a simple Monte Carlo simulation study to compare the accuracy of the two asymptotic approximations. The data is assumed to have unknown mean so that the data is mean-corrected before estimating $f(\omega)$ (this is case (ii) from above). We consider the data generating process

$$
\begin{aligned}
y_{t} & =\mu+u_{t}, \\
u_{t} & =\rho u_{t-1}+\xi_{t}+\theta \xi_{t-1}, \\
\xi_{t} & \sim \text { iid } N(0,1),
\end{aligned}
$$

where $u_{0}=\xi_{0}=0$. The spectral density for $y_{t}$ is given by

$$
f(\omega)=\frac{1}{2 \pi} \frac{\left(1+2 \theta \cos (\omega)+\theta^{2}\right)}{\left(1-2 \rho \cos (\omega)+\rho^{2}\right)} .
$$

Data was generated according to (8) and $\widehat{f}(\omega)$ was computed using (2). Because $\widehat{f}(\omega)$ is exactly invariant to $\mu$, we set $\mu=0$ in all the simulations. Using $R=5,000$ replications we computed the empirical cumulative distribution function, $\widehat{\operatorname{Prob}}(x)$ of $\widehat{f}(\omega) / f((\omega)$ using

$$
\widehat{\operatorname{Pr}} \operatorname{cob}(x)=\frac{1}{R} \sum_{i=1}^{R} \mathbf{1}\left(\frac{\widehat{f}_{i}(\omega)}{f(\omega)}<x\right),
$$

where $\widehat{f}_{1}(\omega), \widehat{f}_{2}(\omega), \ldots, \widehat{f}_{R}(\omega)$ are the simulated realizations from $\widehat{f}(\omega)$ and $1(\cdot)$ is the indicator function. We obtained results for large range of values for $M, \omega, \rho, \theta$ and a group of well known kernels that includes the Bartlett, Parzen, Daniell and quadratic spectral (QS) kernels. 
We report results for $\operatorname{AR}(1)$ errors with $\rho=0.0,0.4,-0.4$, for the frequencies $\omega=0.0,0.5 \pi$, and $b=M / T=0.04,0.2,0.5$. Plots of the empirical CDFs for $T=50,100,200$ are given in Figures 1.1-4.18. The figures are organized first by kernel: Bartlett (Figures 1.1-1.18), Parzen (Figures 2.1-2.18), Daniell (Figures 3.1-3.18), QS (Figures 4.1-4.18) and then by frequency: $\omega=0$ (Figures x.1-x.9) and $\omega=0.5 \pi$ and (Figures x.10-x.18). Each figure also provides plots of the CDFs given by the normal asymptotic approximation from (3) and by the fixed- $b$ approximation from Theorem 1 . The CDFs of the asymptotic random variables in Theorem 1 were accurately estimated using simulation methods. The standard Brownian motions were approximated by scaled partial sums of $i . i . d$. standard normal random variables using 1,000 increments and 50,000 replications.

Figures x.1-x.3 provide results for i.i.d. errors at frequency zero. When a small bandwidth is used $(b=0.04)$ as in Figures $\mathbf{x} .1$, we see that the finite sample empirical CDFs are similar for all three sample sizes and are close to the fixed- $b$ asymptotic CDF. The asymptotic normal CDF, on the other hand, is obviously different between the 0.1 and 0.9 percentiles. In situations where larger bandwidths are used as in Figures x.2 $(b=0.2)$ and x.3 $(b=0.5)$, the fixed- $b$ asymptotic CDF remains very close to the empirical CDFs whereas the asymptotic normal CDF systematically becomes a less accurate approximation. When the data has serial correlation as is the case in Figures x.4-x.6 $(\rho=-0.4)$ and Figures x.7-x.9 $(\rho=0.4)$, the fixed- $b$ asymptotic approximation remains accurate for $b=0.2,0.5$ whereas the asymptotic normal approximation exhibits substantial inaccuracy. For $b=0.04$, neither asymptotic approximation is accurate for $T=50$. But, the fixed- $b$ asymptotic approximation improves as $T$ increases whereas the asymptotic normal approximation remains less accurate. It is interesting to note that for $b=0.2,0.5$ the CDF for the asymptotic normal approximation is strictly positive at the origin. Thus, the asymptotic normal approximation attributes negative values to $\widehat{f}(\omega) / f((\omega)$ with positive probability even though $\widehat{f}(\omega) / f((\omega)$ is positive by construction for the four kernels considered here. This problem does not arise with the fixed- $b$ approximation.

Now consider the frequency $\omega=0.5 \pi$. As Figures x.10-x.18 illustrate, the differences between the normal and fixed- $b$ approximations are smaller than for $\omega=0$ although the patterns are similar. For $b=0.04$ the CDFs of the two asymptotic approximations are very close to each other and they are reasonably close to the empirical CDFs for $T=100,200$. 
For $T=50$ and $\rho \neq 0$ neither asymptotic approximation is adequate. For $b=0.2,0.5$ the fixed- $b$ asymptotic approximation is good for all three sample sizes and all three values of $\rho$ whereas the asymptotic normal approximation is much less accurate.

The overall picture that emerges from the figures is that the fixed- $b$ approximation can be systematically better than the asymptotic normal approximation regardless of the kernel being used. The next section provides some calculations that can shed some light on patterns exhibited in the simulations.

\section{Asymptotic Bias and Variance}

Many of the patterns seen in the simulations can be intuitively explained by examining the asymptotic bias and variances implied by fixed- $b$ asymptotics in comparison to the asymptotic normal approximation. We continue to focus on the case where the data is mean-corrected and the for the sake of concreteness we isolate attention on the Bartlett kernel. In a recent paper, Hashimzade, Kiefer and Vogelsang (2005) formally calculated the following results under fixed- $b$ asymptotics. For $0<\omega<\pi$

$$
\begin{aligned}
\lim _{T \rightarrow \infty} \operatorname{bias}\left[\frac{\widehat{f}(\omega)}{f(\omega)}\right] & =0 \\
\lim _{T \rightarrow \infty} \operatorname{var}\left[\frac{\widehat{f}(\omega)}{f(\omega)}\right] & =b \int_{-1 / b}^{1 / b}(1-b|x|) k^{2}(x) d x=b \int_{-1}^{1}(1-b|x|)(1-|x|)^{2} d x \\
& =2 b\left(\frac{1}{3}-\frac{1}{12} b\right)
\end{aligned}
$$

Note that this variance formula exactly matches the formula obtained by Neave (1970). For $\omega=0$, the formulae are given by

$$
\begin{aligned}
& \lim _{T \rightarrow \infty} \operatorname{bias}\left[\frac{\widehat{f}(\omega)}{f(\omega)}\right]=-b+\frac{1}{3} b^{2} \\
& \lim _{T \rightarrow \infty} \operatorname{var}\left[\frac{\widehat{f}(\omega)}{f(\omega)}\right]=\frac{4}{3} b-\frac{7}{3} b^{2}+\frac{14}{15} b^{3}+\frac{2}{9} b^{4}-\frac{1}{15 b^{2}}(2 b-1)^{5} 1\left(b>\frac{1}{2}\right) .
\end{aligned}
$$

This variance formula differs from $4 b\left(\frac{1}{3}-\frac{1}{12} b\right)$, the formula given by Neave (1970), because Neave's zero frequency variance formula is only valid when the data has mean zero and the data is not mean-corrected. Neave's variance formula and the fixed- $b$ variance are the same at frequency zero when the data is mean zero and is not mean-corrected. 
It is instructive to compare these bias and variance formulae with the well known standard formulae for the Bartlett kernel which are given by

$$
\begin{array}{r}
\lim _{T \rightarrow \infty} \text { bias }\left[\frac{\widehat{f}(\omega)}{f(\omega)}\right]=0, \\
\lim _{T \rightarrow \infty} b^{-1} \operatorname{var}\left[\frac{\widehat{f}(\omega)}{f(\omega)}\right]=\frac{2}{3},
\end{array}
$$

for $0<\omega<\pi$ and

$$
\begin{gathered}
\lim _{T \rightarrow \infty} \text { bias }\left[\frac{\widehat{f}(\omega)}{f(\omega)}\right]=0 \\
\lim _{T \rightarrow \infty} b^{-1} \operatorname{var}\left[\frac{\widehat{f}(\omega)}{f(\omega)}\right]=\frac{4}{3},
\end{gathered}
$$

for $\omega=0$. Under the standard approach, $\operatorname{var}(\widehat{f}(\omega) / f(\omega))$ is approximated by $\frac{2}{3} b$ and $\frac{4}{3} b$ respectively and these quantities match the first terms in the variance approximations given by fixed- $b$ asymptotics. Because $b=\frac{M}{T}$, both asymptotic theories approximate $\operatorname{var}(\widehat{f}(\omega) / f(\omega))$ with a polynomial in $\frac{M}{T}$. The standard approximation is a first order polynomial whereas the fixed- $b$ approximation is a higher order polynomial with the same first order term. When $\frac{M}{T}$ is small, we would expect the higher order terms to be relatively small and this suggests the standard and fixed- $b$ approximations will be similar at least with respect to bias and variance. In light of this observation, it not surprising that the cdfs of the two asymptotic approximations are very close to each other in the figures for $b=0.04$. When $\frac{M}{T}$ is not close to zero, the higher order terms in the fixed- $b$ approximations matter and we would expect the approximations to be different. This is precisely what we see in the figures for $b=0.2,0.5$.

While the above bias and variance formulae help explain the differences between the accuracy of the normal asymptotic approximation and the fixed- $b$ asymptotic approximation, they do not explain the relative accuracy of the fixed- $b$ asymptotic approximation across different values of $b$ and $T$. To provide an explanation, it is useful to examine the wellknown spectral bias formulae derived by Parzen (1957). For the Bartlett and Daniell kernels the Parzen bias is given by $-f^{(1)}(\omega) / M$ and $-f^{(2)}(\omega) \pi^{2} /\left(6 M^{2}\right)$ respectively where

$$
f^{(k)}(\omega)=\frac{1}{2 \pi}\left[\sum_{j=-\infty}^{\infty}|j|^{k} \gamma_{j} \cos (\omega j)\right] .
$$


Because under both the normal asymptotic approximation and the fixed- $b$ asymptotic approximation, $M \rightarrow \infty$ as $T \rightarrow \infty$, the Parzen bias is $o(1)$ and does not appear in the asymptotic approximations (at least to first order). The behavior of the Parzen bias term can explain why the fixed- $b$ approximation improves as $b$ increases when the data is not i.i.d. especially for $T=50$. Given $T$, as $b$ increases, $M$ increases thus reducing the Parzen bias and improving the approximation. This is why in Figures 6 and $9(b=0.5)$ we see that the $T=50 \mathrm{cdf}$ and the fixed- $b$ cdf are close whereas in Figures 4 and $7(b=0.04)$ the $T=50$ cdf and the fixed- $b$ cdf are not close. The Parzen bias also explains why, for a given value of $b$, the fixed- $b$ approximation improves as $T$ increases. With $b$ fixed, as $T$ increases, so does $M$ and the Parzen bias shrinks.

\section{Appendix: Proofs}

Define the following partial sums:

$$
\begin{aligned}
& \widehat{S}_{t}^{c}(\omega)=\sum_{j=1}^{t} \widehat{u}_{j} \cos (\omega j), \\
& \widehat{S}_{t}^{s}(\omega)=\sum_{j=1}^{t} \widehat{u}_{j} \sin (\omega j) .
\end{aligned}
$$

Note that

$$
\widehat{S}_{t}^{s}(0)=\widehat{S}_{t}^{s}(\pi)=0
$$

because $\sin (0)=\sin (\pi j)=0$. Consider the case where $\widehat{d}_{t}=\widehat{\mu}+\widehat{\beta} t$. Then, from simple algebra it follows that $\widehat{u}_{t}=u_{t}-(\widehat{\mu}-\mu)-(\widehat{\beta}-\beta) t$. Because $T^{1 / 2}(\widehat{\mu}-\mu)=O_{p}(1)$ and $T^{3 / 2}(\widehat{\beta}-\beta)=O_{p}(1)$, it is easy to show that for $0<\omega<\pi$,

$$
\begin{aligned}
& T^{-1 / 2} \sum_{t=1}^{[r T]}[(\widehat{\mu}-\mu)-(\widehat{\beta}-\beta) t] \cos (\omega t)=O_{p}\left(T^{-1}\right), \\
& T^{-1 / 2} \sum_{t=1}^{[r T]}[(\widehat{\mu}-\mu)-(\widehat{\beta}-\beta) t] \sin (\omega t)=O_{p}\left(T^{-1}\right),
\end{aligned}
$$

and

$$
T^{-1 / 2} \sum_{t=1}^{[r T]}[(\widehat{\mu}-\mu)-(\widehat{\beta}-\beta) t](-1)^{t}=O_{p}\left(T^{-1}\right) .
$$


Therefore, it directly follows from (5) - (7) that

$$
\begin{gathered}
T^{-1 / 2} \widehat{S}_{[r T]}^{c}(\pi) \Rightarrow \sqrt{2 \pi f(\pi)} W^{*}(r), \\
T^{-1 / 2} \widehat{S}_{[r T]}^{c}(\omega) \Rightarrow \sqrt{\pi f(\omega)} W_{1}(r), \text { for } 0<\omega<\pi, \\
T^{-1 / 2} \widehat{S}_{[r T]}^{s}(\omega) \Rightarrow \sqrt{\pi f(\omega)} W_{2}(r), \text { for } 0<\omega<\pi .
\end{gathered}
$$

Obviously, the limits (10), (11) and (12) continue to hold for $\widehat{d}_{t}=\bar{y}$ or $\widehat{d}_{t}=0$. The remaining case is $\widehat{S}_{t}^{c}(0)=\sum_{j=1}^{t} \widehat{u}_{j}$, and this partial sum has a different type of limit because

$$
T^{-1 / 2} \sum_{t=1}^{[r T]}[(\widehat{\mu}-\mu)-(\widehat{\beta}-\beta) t]=O_{p}(1) .
$$

Simple algebra gives

$$
\begin{aligned}
T^{-1 / 2} \widehat{S}_{[r T]}^{c}(0) & =T^{-1 / 2} \sum_{t=1}^{[r T]} \widehat{u}_{[r T]}=T^{-1 / 2} \sum_{t=1}^{[r T]}\left[u_{t}-(\widehat{\mu}-\mu)-(\widehat{\beta}-\beta) t\right] \\
& =T^{-1 / 2} \sum_{t=1}^{[r T]} u_{t}-\frac{[r T]}{T} T^{1 / 2}(\widehat{\mu}-\mu)-T^{3 / 2}(\widehat{\beta}-\beta) \frac{1}{T^{2}} \sum_{t=1}^{[r T]} t \\
& \Rightarrow \sqrt{2 \pi f(0)} \widehat{W}(r) .
\end{aligned}
$$

Note that the limiting result (13) is a standard result that follows from (4) and simplifies in obvious ways for $\widehat{d}_{t}=\bar{y}$ or $\widehat{d}_{t}=0$.

Proof of Theorem 1. Define the following functions:

$$
\begin{aligned}
K_{i j} & =k\left(\frac{i-j}{b T}\right)=k^{*}\left(\frac{i-j}{T}\right), \\
\Delta^{2} K_{i j} & =\left(K_{i j}-K_{i, j+1}\right)-\left(K_{i+1, j}-K_{i+1, j+1}\right) .
\end{aligned}
$$


Simple algebra gives

$$
\begin{aligned}
\widehat{f}(\omega)= & \frac{1}{2 \pi}\left[\widehat{\gamma}_{0}+2 \sum_{j=1}^{T-1} k\left(\frac{j}{b T}\right) \widehat{\gamma}_{j} \cos (\omega j)\right] \\
= & \frac{1}{2 \pi} T^{-1} \sum_{i=1}^{T} \sum_{j=1}^{T} \widehat{u}_{i} \widehat{u}_{j} k\left(\frac{i-j}{b T}\right) \cos [\omega(i-j)] \\
= & \frac{1}{2 \pi} T^{-1} \sum_{i=1}^{T} \sum_{j=1}^{T} \widehat{u}_{i} \cos (\omega i) K_{i j} \widehat{u}_{j} \cos (\omega j)+ \\
& +\frac{1}{2 \pi} T^{-1} \sum_{i=1}^{T} \sum_{j=1}^{T} \widehat{u}_{i} \sin (\omega i) K_{i j} \widehat{u}_{j} \sin (\omega j)
\end{aligned}
$$

Rewrite the first term in (14) as the following:

$$
\begin{aligned}
\frac{1}{2 \pi} T^{-1} \sum_{i=1}^{T} \sum_{j=1}^{T} \widehat{u}_{i} \cos (\omega i) K_{i j} \widehat{u}_{j} \cos (\omega j) & =\frac{1}{2 \pi} T^{-1} \sum_{i=1}^{T} \widehat{u}_{i} \cos (\omega i) \sum_{j=1}^{T} K_{i j} \widehat{u}_{j} \cos (\omega j) \\
& =\frac{1}{2 \pi} T^{-1} \sum_{i=1}^{T} a_{i} b_{i}
\end{aligned}
$$

where

$$
a_{i}=\widehat{u}_{i} \cos (\omega i), \quad b_{i}=\sum_{j=1}^{T} K_{i j} \widehat{u}_{j} \cos (\omega j)
$$

Using the identity

$$
\sum_{i=1}^{T} a_{i} b_{i}=\sum_{i=1}^{T-1}\left[\left(b_{i}-b_{i+1}\right) \sum_{j=1}^{i} a_{j}\right]+b_{T} \sum_{j=1}^{T} a_{j}
$$

we obtain, for $a_{i}$ and $b_{i}$ defined above,

$$
\sum_{i=1}^{T} a_{i} b_{i}=\sum_{i=1}^{T-1}\left(\sum_{j=1}^{T}\left(K_{i j}-K_{i+1, j}\right) \widehat{u}_{j} \cos (\omega j) \widehat{S}_{i}^{c}(\omega)\right)+\sum_{j=1}^{T} K_{T j} \widehat{u}_{j} \cos (\omega j) \widehat{S}_{T}^{c}(\omega) .
$$

By applying the identity (15) one more time, we obtain for the first term in (16)

$$
\begin{aligned}
\sum_{j=1}^{T}\left(K_{i j}-K_{i+1, j}\right) \widehat{u}_{j} \cos (\omega j) & =\sum_{j=1}^{T-1}\left[\left(K_{i j}-K_{i+1, j}\right)-\left(K_{i, j+1}-K_{i+1, j+1}\right)\right] \widehat{S}_{j}^{c}(\omega) \\
& +\left(K_{i T}-K_{i+1, T}\right) \widehat{S}_{T}^{c}(\omega) \\
& =\sum_{j=1}^{T-1} \Delta^{2} K_{i j} \widehat{S}_{j}^{c}(\omega)+\left(K_{i T}-K_{i+1, T}\right) \widehat{S}_{T}^{c}(\omega) .
\end{aligned}
$$


Similarly, for the second term in (16) we obtain

$$
\sum_{j=1}^{T} K_{T j} \widehat{u}_{j} \cos (\omega j)=\sum_{j=1}^{T-1}\left(K_{T j}-K_{T, j+1}\right) \widehat{S}_{j}^{c}(\omega)+K_{T T} \widehat{S}_{T}^{c}(\omega) .
$$

Finally, noting that $K_{i j}=K_{j i}$ and $K_{T T}=k(0)=1$, we obtain the following expression for the first term in (14)

$$
\begin{gathered}
\frac{1}{2 \pi} T^{-1} \sum_{i=1}^{T} \sum_{j=1}^{T} \widehat{u}_{i} \cos (\omega i) K_{i j} \widehat{u}_{j} \cos (\omega j)= \\
\frac{1}{2 \pi} T^{-1} \sum_{i=1}^{T-1} T^{-1} \sum_{j=1}^{T-1} T^{-1 / 2} \widehat{S}_{i}^{c}(\omega) T^{2} \Delta^{2} K_{i j} T^{-1 / 2} \widehat{S}_{j}^{c}(\omega) \\
+2 \frac{1}{2 \pi} T^{-1} \sum_{j=1}^{T} T^{-1 / 2} \widehat{S}_{j}^{c}(\omega) T\left(K_{j T}-K_{j+1, T}\right) T^{-1 / 2} \widehat{S}_{T}^{c}(\omega)+\frac{1}{2 \pi}\left(T^{-1 / 2} \widehat{S}_{T}^{c}(\omega)\right)^{2} .
\end{gathered}
$$

Define

$$
\begin{gathered}
D_{1 T}^{*}(r)=T\left[k^{*}\left(\frac{[r T]+1}{T}\right)-k^{*}\left(\frac{[r T]}{T}\right)\right] \\
D_{2 T}^{*}(r)=T^{2}\left[\left(k^{*}\left(\frac{[r T]+1}{T}\right)-k^{*}\left(\frac{[r T]}{T}\right)\right)-\left(k^{*}\left(\frac{[r T]}{T}\right)-k^{*}\left(\frac{[r T]-1}{T}\right)\right)\right] .
\end{gathered}
$$

Notice that $T\left(K_{j+1, T}-K_{j T}\right)=D_{1 T}^{*}(r)$ and $T^{2} \Delta^{2} K_{i j}=-D_{2 T}^{*}\left(\frac{i-j}{T}\right)$ which enables us to write (17) as

$$
\begin{gathered}
\frac{1}{2 \pi} T^{-1} \sum_{i=1}^{T} \sum_{j=1}^{T} \widehat{u}_{i} \cos (\omega i) K_{i j} \widehat{u}_{j} \cos (\omega j)= \\
-\frac{1}{2 \pi} \int_{0}^{1} \int_{0}^{1} T^{-1 / 2} \widehat{S}_{[r T]}^{c}(\omega) D_{2 T}^{*}(r-s) T^{-1 / 2} \widehat{S}_{[s T]}^{c}(\omega) d r d s \\
+2 \frac{1}{2 \pi} \int_{0}^{1} T^{-1 / 2} \widehat{S}_{[r T]}^{c}(\omega) D_{1 T}^{*}(r) T^{-1 / 2} \widehat{S}_{T}^{c}(\omega) d r+\frac{1}{2 \pi}\left(T^{-1 / 2} \widehat{S}_{T}^{c}(\omega)\right)^{2} .
\end{gathered}
$$

If $k^{\prime \prime}(x)$ exists and is continuous, then

$$
\begin{aligned}
& \lim _{T \rightarrow \infty} D_{1 T}^{*}(r)=k^{* \prime}(r) \\
& \lim _{T \rightarrow \infty} D_{2 T}^{*}(r)=k^{* \prime \prime}(r)
\end{aligned}
$$

by definitions of the first and second derivatives. Because (18) is a continuous function of $T^{-1 / 2} \widehat{S}_{[r T]}^{c}(\omega), D_{1 T}^{*}(r)$ and $D_{2 T}^{*}(r)$, its limit can be obtained using the continuous mapping 
theorem (Billingsley (1968, Theorem 5.1) along with (19), (20) and the appropriate limit of $T^{-1 / 2} \widehat{S}_{[r T]}^{c}(\omega)$. For $\omega>0$ the limit of $T^{-1 / 2} \widehat{S}_{[r T]}^{c}(\omega)$ follows from (11) for $\omega<\pi$ and from (10) for $\omega=\pi$. For $\omega=0$, (17) simplifies using the fact that $\widehat{S}_{T}^{c}(0)=0$ and the limit of $T^{-1 / 2} \widehat{S}_{[r T]}^{c}(\omega)$ follows from (13). The second term in (14) is identical to (17), with $\widehat{S}_{i}^{c}(\omega)$ replaced by $\widehat{S}_{i}^{s}(\omega)$ and for $0<\omega<\pi$ the limit follow using analogous arguments. For $\omega=0, \pi$ the second term vanishes because of (9). This completes the proof of part 1 of the theorem.

If $k(x)$ is not everywhere twice continuously differentiable, we cannot apply (20) and (19) directly. The leading cases are kernels that truncate, i.e. kernels that put zero weight on sample autocovariances with lag greater than $M$. Suppose $k(x)=0$ for $|x| \geq 1, k(x)$ is continuous, and $k(x)$ is twice continuously differentiable everywhere except for possibly $|x|=1$. We rewrite the sums in (17) to reflect the truncation in $k(x)$. For the first term in (17) we have:

$$
\begin{gathered}
T^{-1} \sum_{i=1}^{T-1} \sum_{j=1}^{T-1} \widehat{S}_{i}^{c}(\omega) \Delta^{2} K_{i j} \widehat{S}_{j}^{c}(\omega)= \\
T^{-1} \sum_{|i-j|<b T} \widehat{S}_{i}^{c}(\omega) \Delta^{2} K_{i j} \widehat{S}_{j}^{c}(\omega)+T^{-1} \sum_{i-j=b T} \widehat{S}_{i}^{c}(\omega) \Delta^{2} K_{i j} \widehat{S}_{j}^{c}(\omega) \\
+T^{-1} \sum_{i-j=-b T} \widehat{S}_{i}^{c}(\omega) \Delta^{2} K_{i j} \widehat{S}_{j}^{c}(\omega)+T^{-1} \sum_{|i-j|>b T} \widehat{S}_{i}^{c}(\omega) \Delta^{2} K_{i j} \widehat{S}_{j}^{c}(\omega)
\end{gathered}
$$

By the assumptions listed above, $k(x)$ is continuous and twice continuously differentiable in the range of the first sum and is zero in the range of the last sum. Hence, the limit of the first term follows from the arguments used in the proof of part 1 and the last term vanishes. For the second term we have

$$
T^{-1} \sum_{i-j=b T} \widehat{S}_{i}^{c}(\omega) \Delta^{2} K_{i j} \widehat{S}_{j}^{c}(\omega)=
$$




$$
\begin{aligned}
& T^{-1} \sum_{j=1}^{T-b T} \widehat{S}_{j}^{c}(\omega)\left[\left(K_{j+b T, j}-K_{j+b T, j+1}\right)-\left(K_{j+b T+1, j}-K_{j+b T+1, j+1}\right)\right] \widehat{S}_{j+b T}^{c}(\omega) \\
& =T^{-1} \sum_{j=1}^{T-b T} \widehat{S}_{j}^{c}(\omega)\left[\left(k(1)-k\left(1-\frac{1}{b T}\right)\right)-\left(k\left(1+\frac{1}{b T}\right)-k(1)\right)\right] \widehat{S}_{j+b T}^{c}(\omega) \\
& =\frac{1}{b T} \sum_{j=1}^{T-b T} T^{-1 / 2} \widehat{S}_{j}^{c}(\omega) \frac{k(1)-k\left(1-\frac{1}{b T}\right)}{\frac{1}{b T}} T^{-1 / 2} \widehat{S}_{j+b T}^{c}(\omega) .
\end{aligned}
$$

By definition of the derivative from the left it follows that,

$$
\lim _{T \rightarrow \infty} \frac{k(1)-k\left(1-\frac{1}{b T}\right)}{\frac{1}{b T}}=k_{-}^{\prime}(1) .
$$

Similar manipulation with the third term in (21) yields

$T^{-1} \sum_{i-j=-b T} \widehat{S}_{i}^{c}(\omega) \Delta^{2} K_{i j} \widehat{S}_{j}^{c}(\omega)=-\frac{1}{b T} \sum_{j=1}^{T-b T} T^{-1 / 2} \widehat{S}_{j}^{c}(\omega) \frac{k\left(-1+\frac{1}{b T}\right)-k(-1)}{\frac{1}{b T}} T^{-1 / 2} \widehat{S}_{j+b T}^{c}(\omega)$.

By definition of the derivative from the right, it follows that

$$
\lim _{T \rightarrow \infty} \frac{k\left(-1+\frac{1}{b T}\right)-k(-1)}{\frac{1}{b T}}=k_{+}^{\prime}(-1) .
$$

By symmetry of $k(x)$ around $x=0$, it follows that $k_{+}^{\prime}(-1)=-k_{-}^{\prime}(1)$. Hence, second and third terms in (21) are equal and can be combined into one. Now consider the second term in (17):

$$
\begin{gathered}
T^{-1} \sum_{j=1}^{T} \widehat{S}_{j}^{c}(\omega)\left(K_{j T}-K_{j+1, T}\right) \widehat{S}_{T}^{c}(\omega) \\
=T^{-1} \sum_{j=1}^{T-b T-1} \widehat{S}_{j}^{c}(\omega)\left(K_{j T}-K_{j+1, T}\right) \widehat{S}_{T}^{c}(\omega)+T^{-1} \sum_{j=T-b T}^{T-1} \widehat{S}_{j}^{c}(\omega)\left(K_{j T}-K_{j+1, T}\right) \widehat{S}_{T}^{c}(\omega)
\end{gathered}
$$

The first term in (23) vanishes because for $1 \leq j \leq T-b T-1$ we have $\frac{j-T}{b T}<-1$ and $\frac{j+1-T}{b T} \leq-1$, and therefore $K_{j T}=K_{j+1, T}=0$. The second term in (23) can be expressed as

$$
\begin{aligned}
& T^{-1} \sum_{j=T-b T}^{T-1} \widehat{S}_{j}^{c}(\omega)\left(K_{j T}-K_{j+1, T}\right) \widehat{S}_{T}^{c}(\omega) \\
& =T^{-1} \sum_{j=T-b T}^{T-1} \widehat{S}_{j}^{c}(\omega)\left(K_{j T}-K_{j+1, T}\right) \widehat{S}_{T}^{c}(\omega) \\
& =-\frac{1}{b T} \sum_{j=T-b T}^{T-1} T^{-1 / 2} \widehat{S}_{j}^{c}(\omega) \frac{k\left(\frac{j+1-T}{b T}\right)-k\left(\frac{j-T}{b T}\right)}{\frac{1}{b T}} T^{-1 / 2} \widehat{S}_{T}^{c}(\omega) .
\end{aligned}
$$


By definition of the first derivative,

$$
\lim _{T \rightarrow \infty} \frac{k\left(\frac{j+1-T}{b T}\right)-k(j-T)}{\frac{1}{b T}}=k^{\prime}\left(\frac{r-1}{b}\right)=-k^{\prime}\left(\frac{1-r}{b}\right)
$$

where the last equality follows from the symmetry of $k(x)$. Collecting all terms in (21) and (23) into (17), adding corresponding terms with $\widehat{S}_{j}^{s}(\omega)$ in place of $\widehat{S}_{j}^{c}(\omega)$, and using (22) and (24) along with (10), (11), (12), (13) and (9) (as needed depending on $\omega$ ) establishes part 2 of the theorem.

To prove part 3 of Theorem 1 we use the definition of Bartlett kernel:

$$
K_{i j}=k\left(\frac{i-j}{b T}\right)= \begin{cases}1-\frac{|i-j|}{b T}, & |i-j| \leq b T \\ 0, & |i-j|>b T\end{cases}
$$

Then it follows that

$$
\begin{gathered}
K_{i j}-K_{i, j+1}= \begin{cases}0, & i \leq j-b T \\
\frac{1}{b T}, & j+1-b T \leq i \leq j \\
-\frac{1}{b T}, & j+1 \leq i \leq j+b T \\
0, & i \geq j+b T+1\end{cases} \\
K_{i+1, j}-K_{i+1, j+1}= \begin{cases}0, & i \leq j-b T-1 \\
\frac{1}{b T}, & j-b T \leq i \leq j-1 \\
-\frac{1}{b T}, & j \leq i \leq j-1+b T \\
0, & i \geq j+b T\end{cases}
\end{gathered}
$$

and

$$
\Delta^{2} K_{i j}= \begin{cases}\frac{2}{b T}, & j=i \\ -\frac{1}{b T}, & i=j \pm b T \\ 0 & \text { otherwise }\end{cases}
$$

Hence, for the first term in (17) we obtain

$$
\begin{aligned}
& T^{-1} \sum_{i=1}^{T-1} \sum_{j=1}^{T-1} \widehat{S}_{i}^{c}(\omega) \Delta^{2} K_{i j} \widehat{S}_{j}^{c}(\omega) \\
& =\frac{2}{b T} \sum_{i=1}^{T-1}\left(T^{-1 / 2} \widehat{S}_{i}^{c}(\omega)\right)^{2}-\frac{1}{b T} \sum_{j=1}^{T-[b T]-1} T^{-1 / 2} \widehat{S}_{j+[b T]}^{c}(\omega) T^{-1 / 2} \widehat{S}_{j}^{c}(\omega) \\
& \quad-\frac{1}{b T} \sum_{i=1}^{T-[b T]-1} T^{-1 / 2} \widehat{S}_{i}^{c}(\omega) T^{-1 / 2} \widehat{S}_{i+[b T]}^{c}(\omega) \\
& =\frac{2}{b T} \sum_{i=1}^{T-1}\left(T^{-1 / 2} \widehat{S}_{i}^{c}(\omega)\right)^{2}-\frac{2}{b T} \sum_{i=1}^{T-[b T]-1} T^{-1 / 2} \widehat{S}_{i}^{c}(\omega) T^{-1 / 2} \widehat{S}_{i+[b T]}^{c}(\omega) .
\end{aligned}
$$


Similarly, in the second term of (17) we have

$$
K_{T, j}-K_{T, j+1}= \begin{cases}0, & 1 \leq j \leq T-b T-1 \\ -\frac{1}{b T}, & T-b T \leq j \leq T-1\end{cases}
$$

(given that $j$ takes values from 1 to $T-1$ ). Hence, (17) can be expressed as

$$
\begin{aligned}
\frac{1}{2 \pi} \frac{2}{b T} \sum_{i=1}^{T-1}\left(T^{-1 / 2} \widehat{S}_{i}^{c}(\omega)\right)^{2} & -\frac{1}{2 \pi} T^{-1} \frac{2}{b T} \sum_{i=1}^{T-[b T]-1} T^{-1 / 2} \widehat{S}_{i}^{c}(\omega) T^{-1 / 2} \widehat{S}_{i+[b T]}^{c}(\omega) \\
& -2 \frac{1}{2 \pi} T^{-1} \sum_{j=T-[b T]}^{T-1} T^{-1 / 2} \widehat{S}_{j}^{c}(\omega) T^{-1 / 2} \widehat{S}_{T}^{c}(\omega)+\frac{1}{2 \pi}\left(T^{-1 / 2} \widehat{S}_{T}^{c}\right)^{2} .
\end{aligned}
$$

An analogous expression obtains with $\widehat{S}_{j}^{s}(\omega)$ in place of $\widehat{S}_{j}^{c}(\omega)$ and the limit follows using (10), (11), (12), (13) and (9) (as needed depending on $\omega$ ). This completes the proof of part 3 of the theorem. 


\section{References}

Anderson, T.: (1971), The Statistical Analysis of Time Series, Wiley, New York.

Bartlett, M.: (1950), Periodogram analysis and continuous spectra, Biometrika 37, 1-16.

Billingsley, P.: (1968), Convergence of Probability Measures, Wiley, New York.

Boutahar, M.: (2006), A functional central limit theorem for long memory processes and application to unstable autoregression. Working Paper 2006-16 GREQAM, Université de la Méditerranée, Marseille.

Bunzel, H. and Vogelsang, T. J.: (2005), Powerful trend function tests that are robust to strong serial correlation with an application to the prebisch-singer hypothesis, Journal of Business and Economic Statistics 23, 381-394.

Daniell, P. J.: (1946), Discussion on 'symposium on autocorrelation in time series', Journal of the Royal Statistical Society, Supplement 8, 88-90.

Grenander, U. and Rosenblatt, M.: (1953), Statistical spectral analysis arising from stationary stochastic processes, Annals of Mathematical Statistics 24, 537-558.

Hashimzade, N., Kiefer, N. M. and Vogelsang, T. J.: (2005), Moments of hac robust covariance matrix estimators under fixed- $b$ asymptotics. Working Paper, Department of Economics, Cornell University.

Kiefer, N. M. and Vogelsang, T. J.: (2005), A new asymptotic theory for heteroskedasticityautocorrelation robust tests, Econometric Theory 21, 1130-1164.

Neave, H. R.: (1970), An improved formula for the asymptotic variance of spectrum estimates, Annals of Mathematical Statistics 41, 70-77.

Ng, S. and Perron, P.: (1996), The exact error in estimating the spectral density at the origin, Journal of Time Series Analysis 17, 379-408.

Parzen, E.: (1957), On consistent estimates of the spectrum of a stationary time series, Annals of Mathematical Statistics 28, 329-348. 
Parzen, E.: (1961), Mathematical considerations in the estimation of spectra, Technometrics 3, 167-190.

Phillips, P. C. B., Sun, Y. and Jin, S.: (2005)a, Optimal bandwidth selection in heteroskedasticity-autocorrelation robust testing. Working Paper, Department of Economics, UCSD.

Phillips, P. C. B., Sun, Y. and Jin, S.: (2005)b, Spectral density estimation and robust hypothesis testing using steep origin kernels without truncation, International Economic Review, forthcoming .

Priestley, M. B.: (1981), Spectral Analysis and Time Series, Vol. 1, Academic Press, New York.

Tukey, J. W.: (1949), The sampling theory of power spectrum estimates, Symposium on Applications of Autocorrelation Analysis to Physical Problems, NAVEXOS-P-735, Office of Naval Research, Department of the Navy, Washington, D.C., pp. 47-67. 


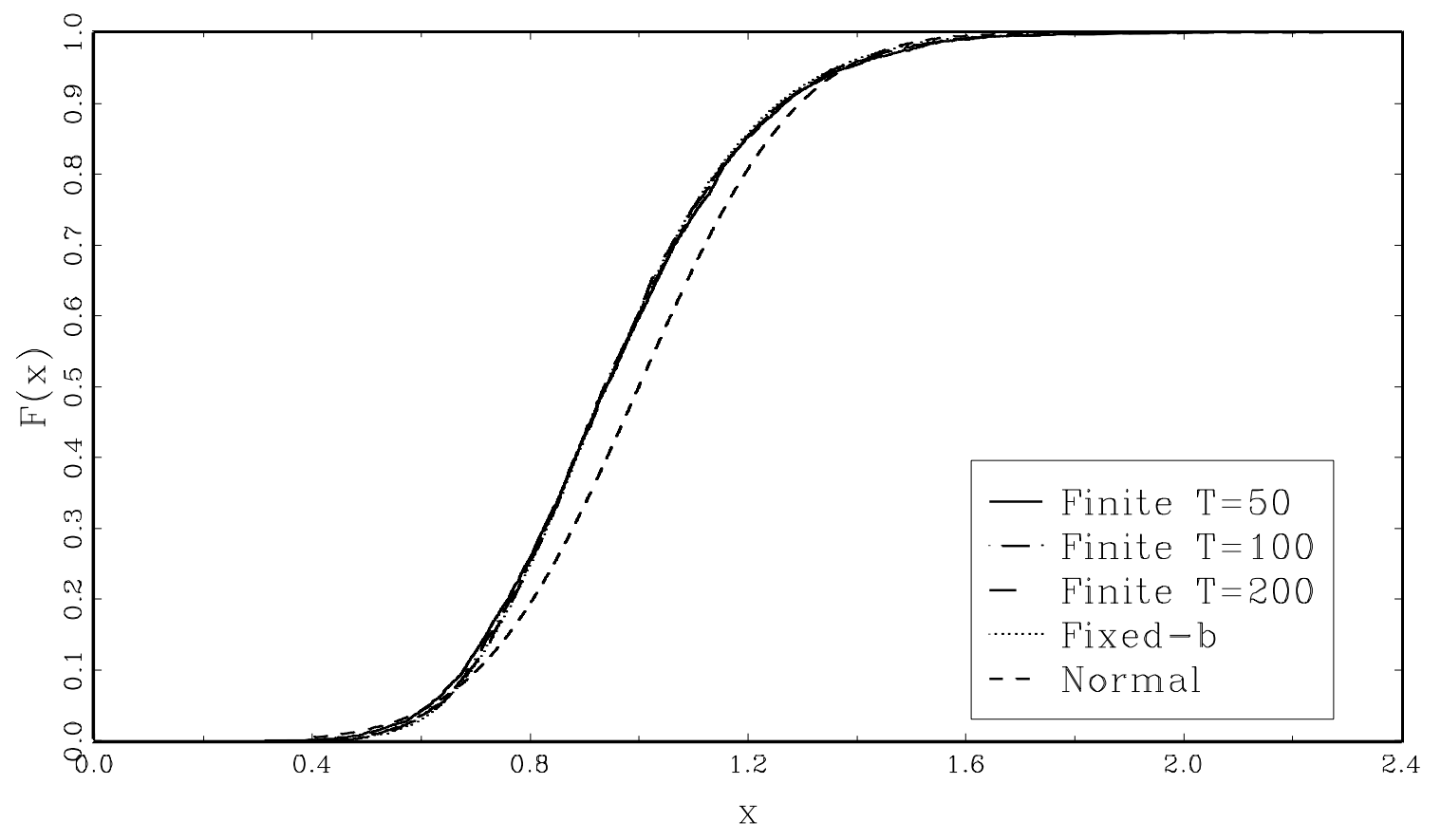

Figure 1.1: CDF Functions, Bartlett Kernel, $\omega=0, \rho=0, b=0.04$

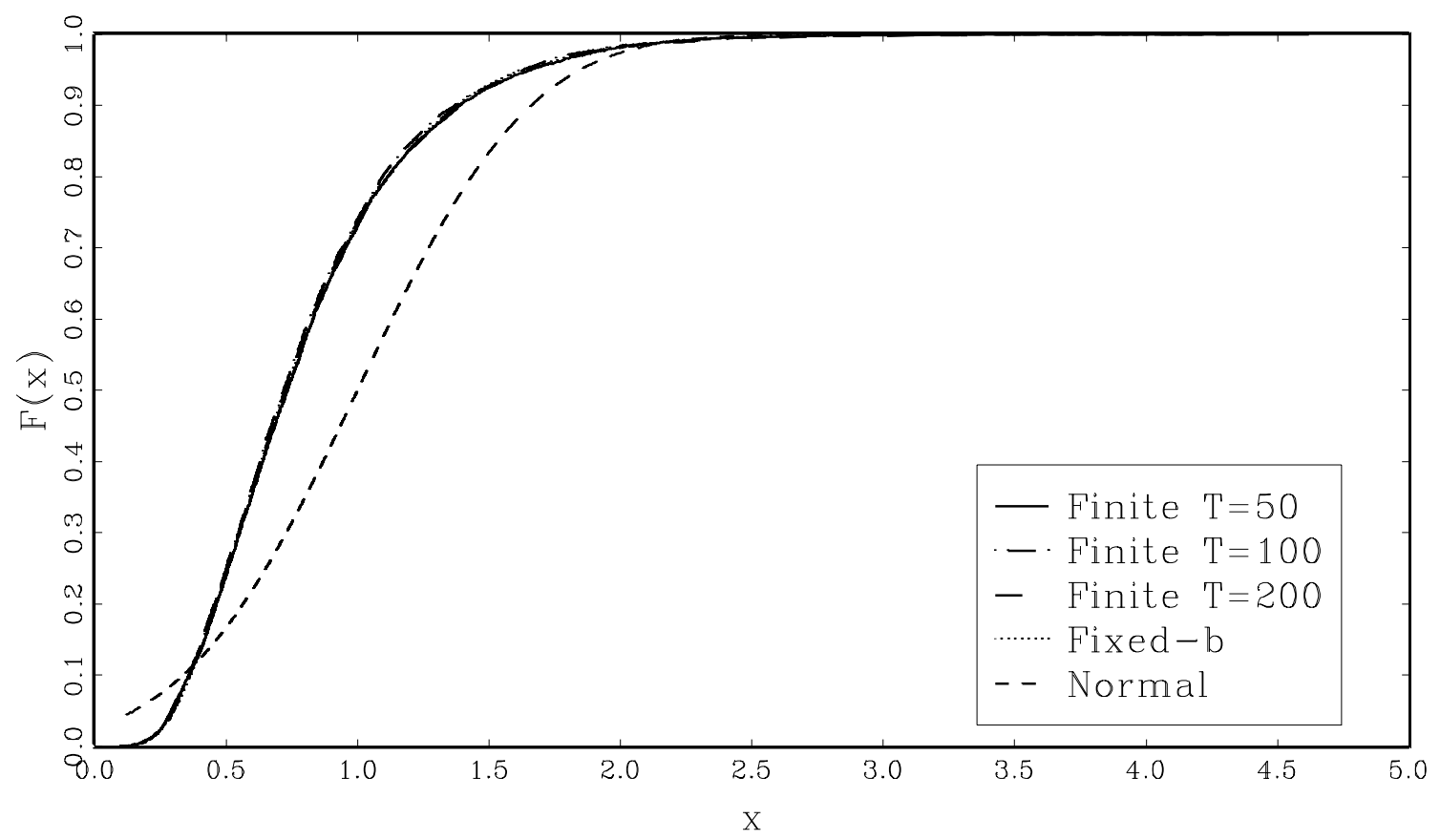

Figure 1.2: CDF Functions, Bartlett Kernel, $\omega=0, \rho=0, b=0.2$ 


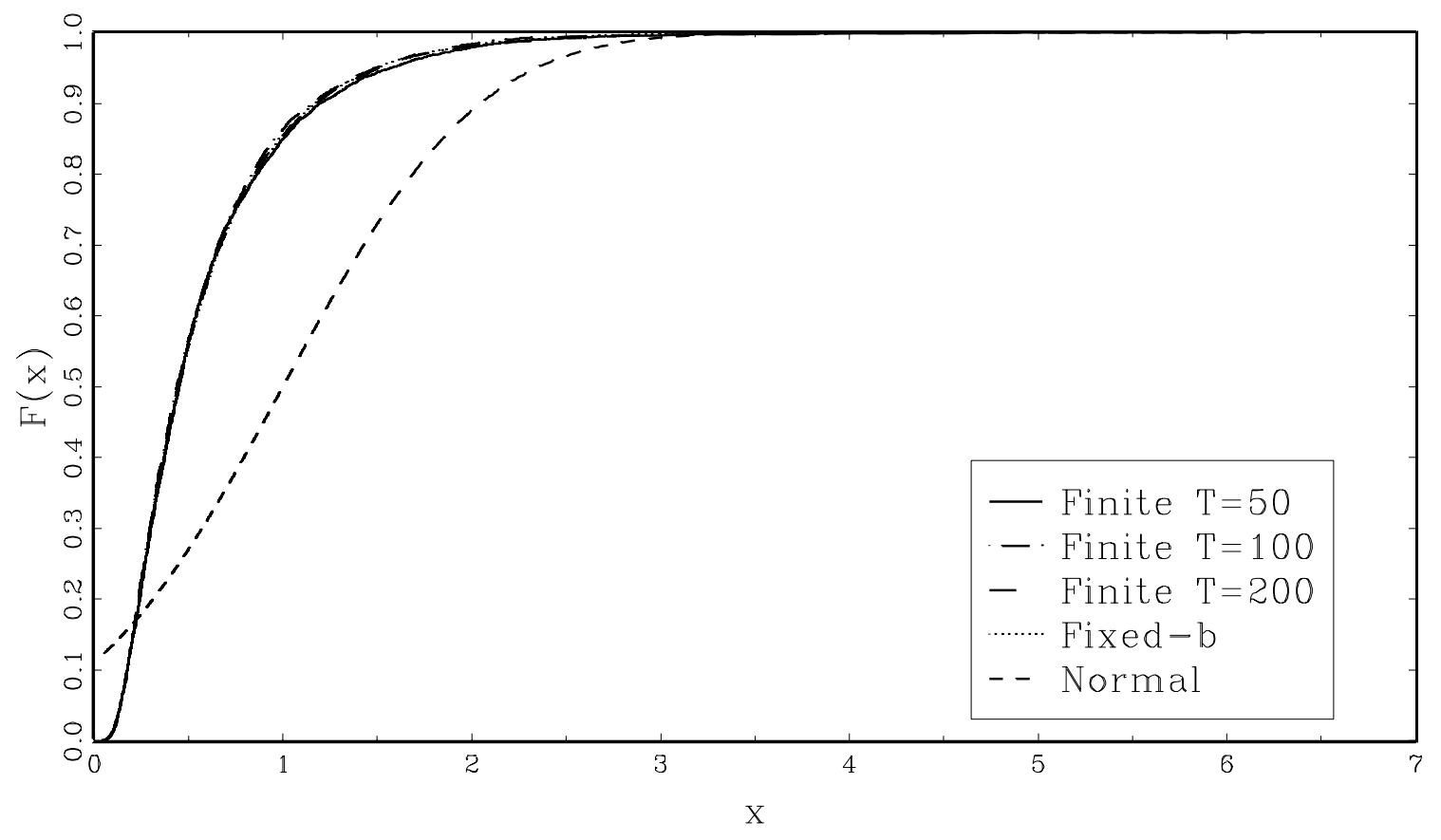

Figure 1.3: CDF Functions, Bartlett Kernel, $\omega=0, \rho=0, b=0.5$

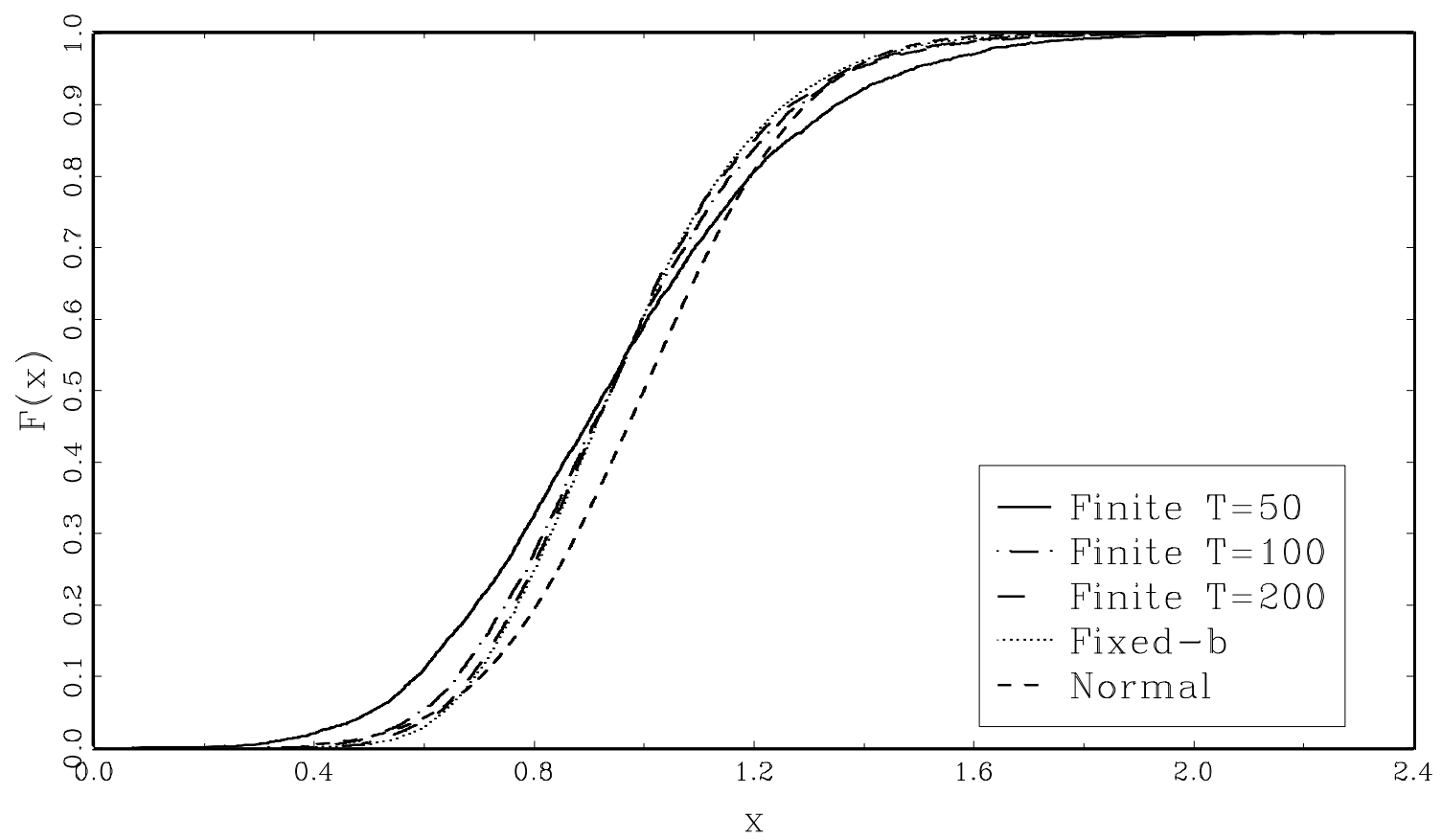

Figure 1.4: CDF Functions, Bartlett Kernel, $\omega=0, \rho=-0.4, \quad b=0.04$ 


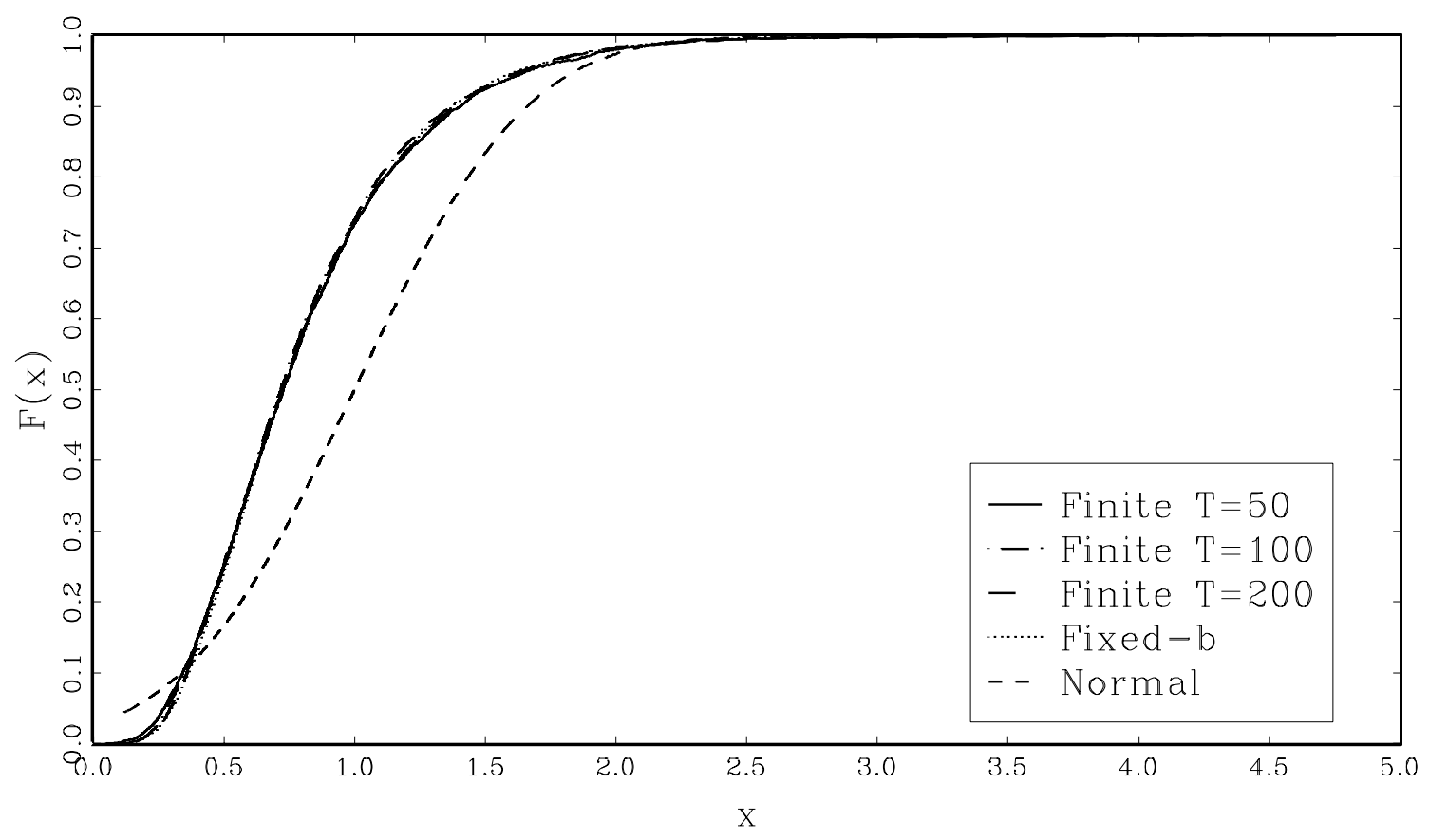

Figure 1.5: CDF Functions, Bartlett Kernel, $\omega=0, \quad \rho=-0.4, \quad b=0.2$

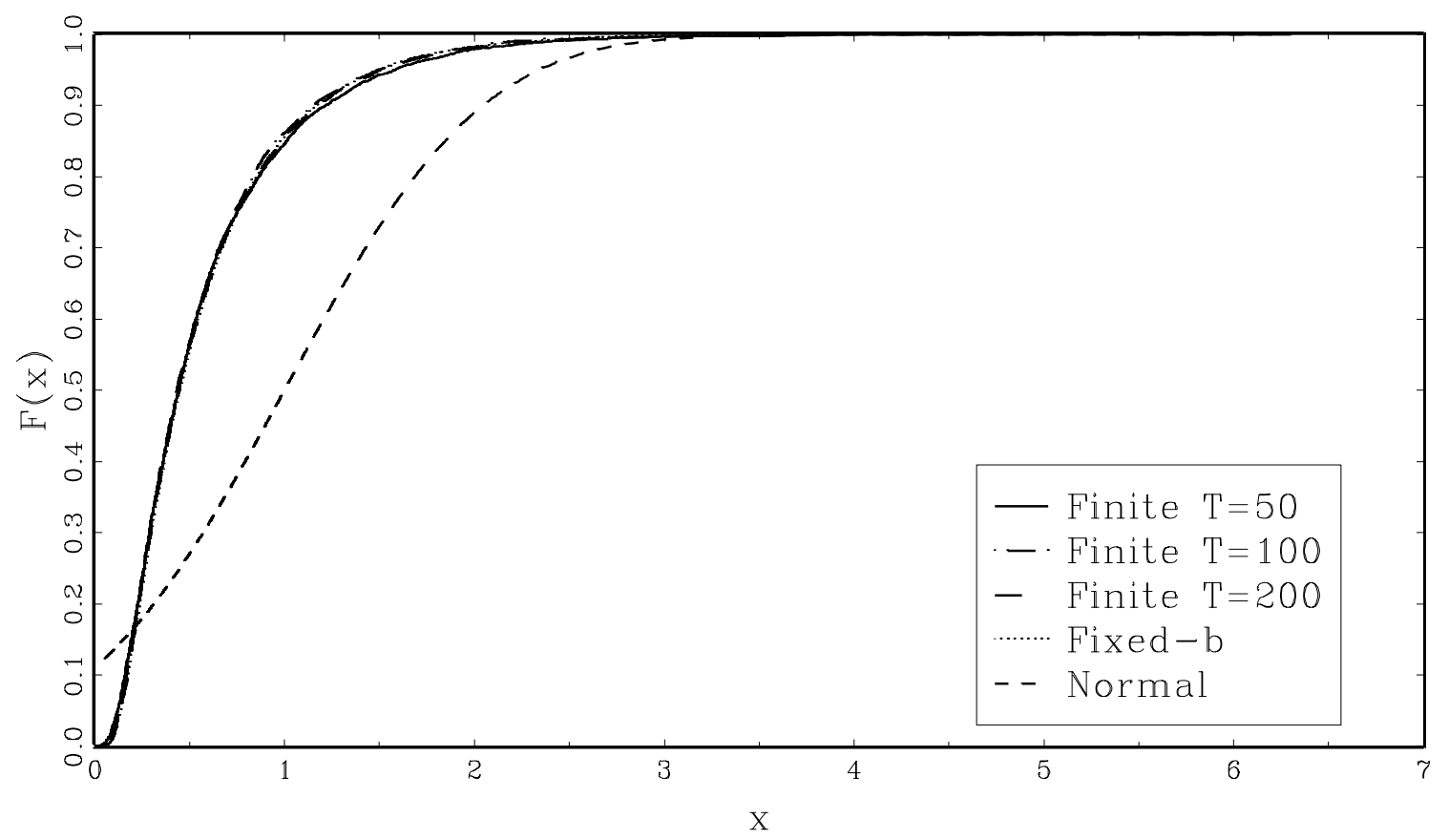

Figure 1.6: CDF Functions, Bartlett Kernel, $\omega=0, \rho=-0.4, \quad b=0.5$ 


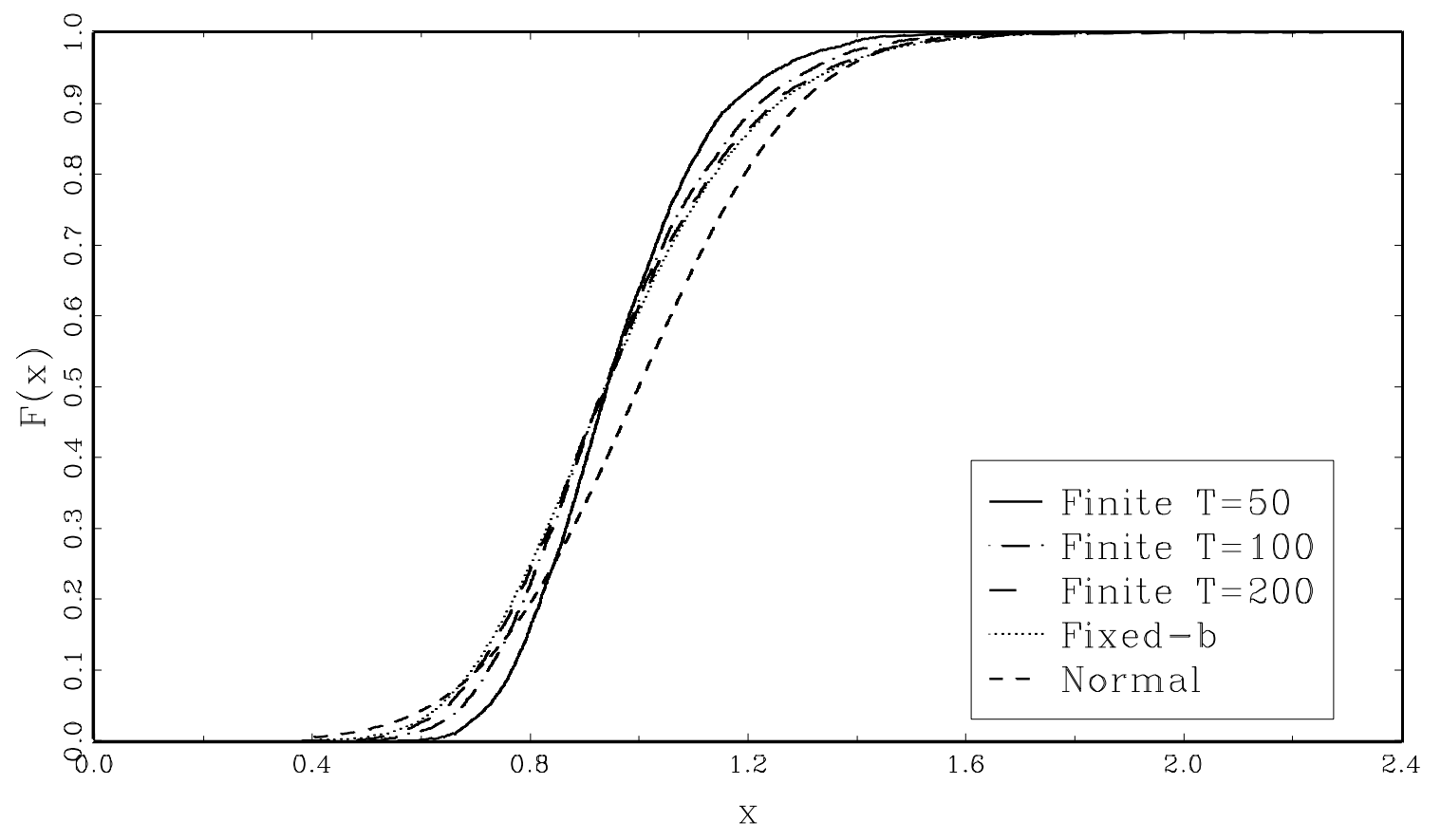

Figure 1.7: CDF Functions, Bartlett Kernel, $\omega=0, \quad p=0.4, \quad b=0.04$

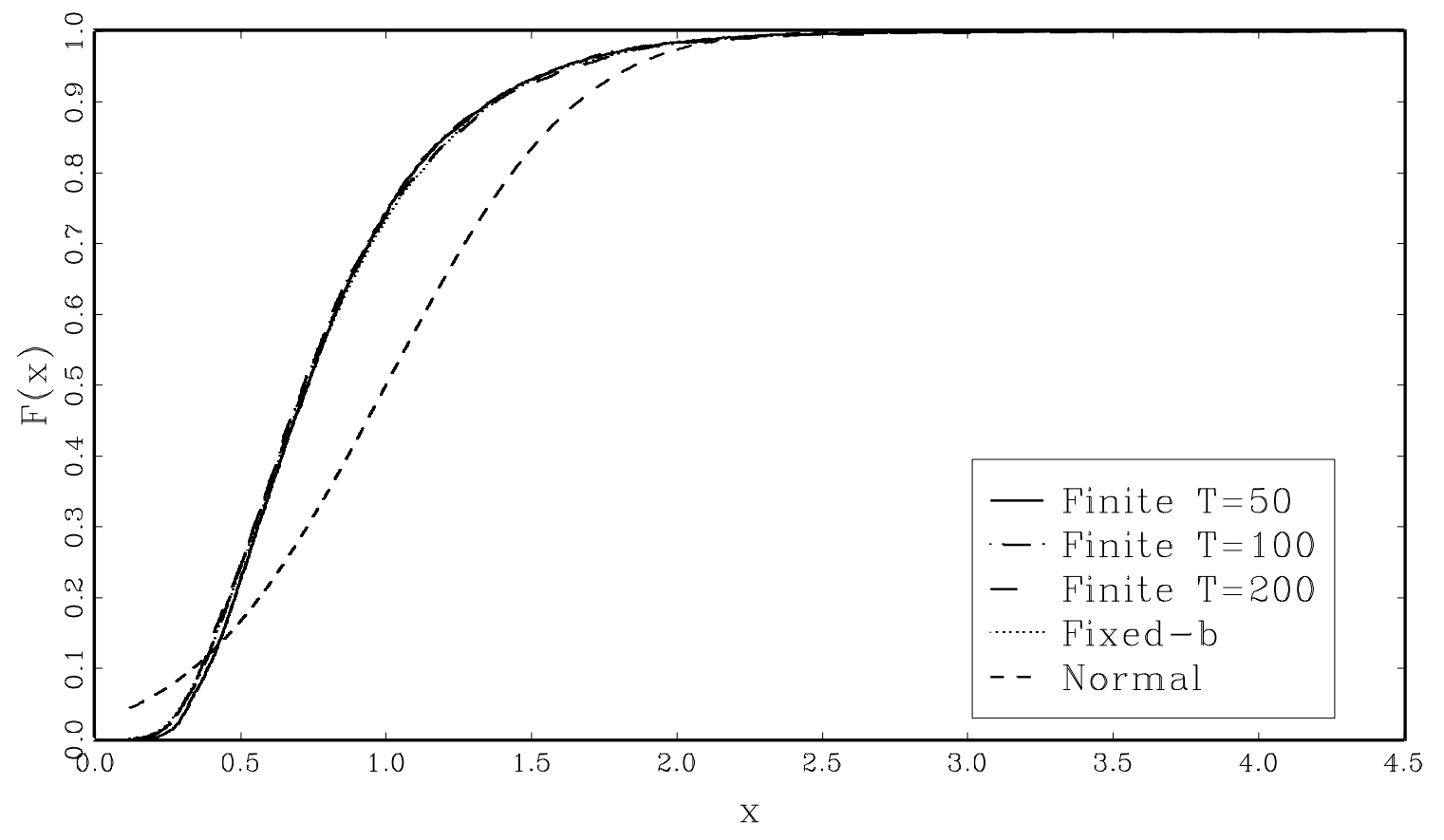

Figure 1.8: CDF Functions, Bartlett Kernel, $\omega=0, \rho=0.4, b=0.2$ 


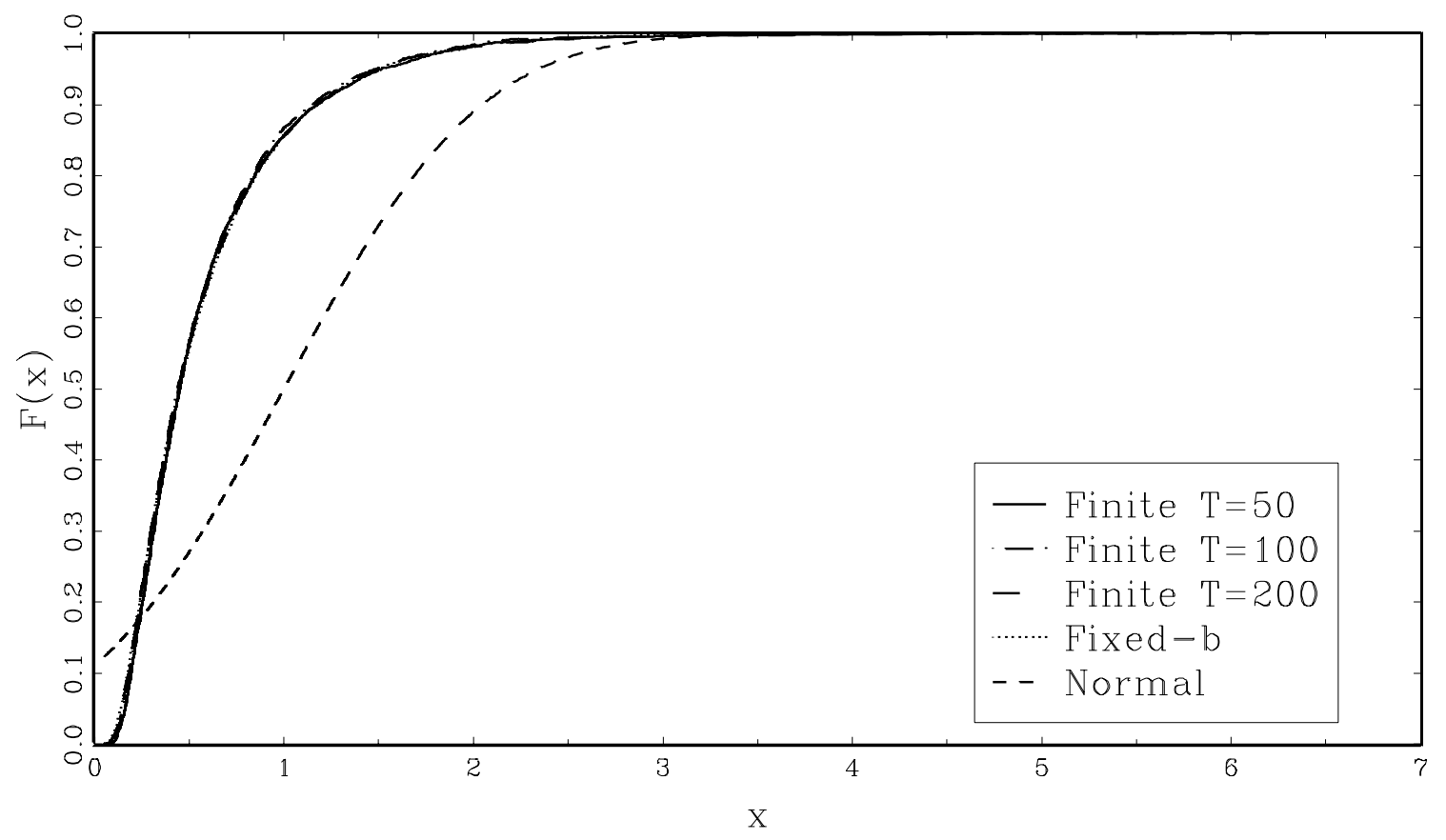

Figure 1.9: CDF Functions, Bartlett Kernel, $\omega=0, \rho=0.4, b=0.5$

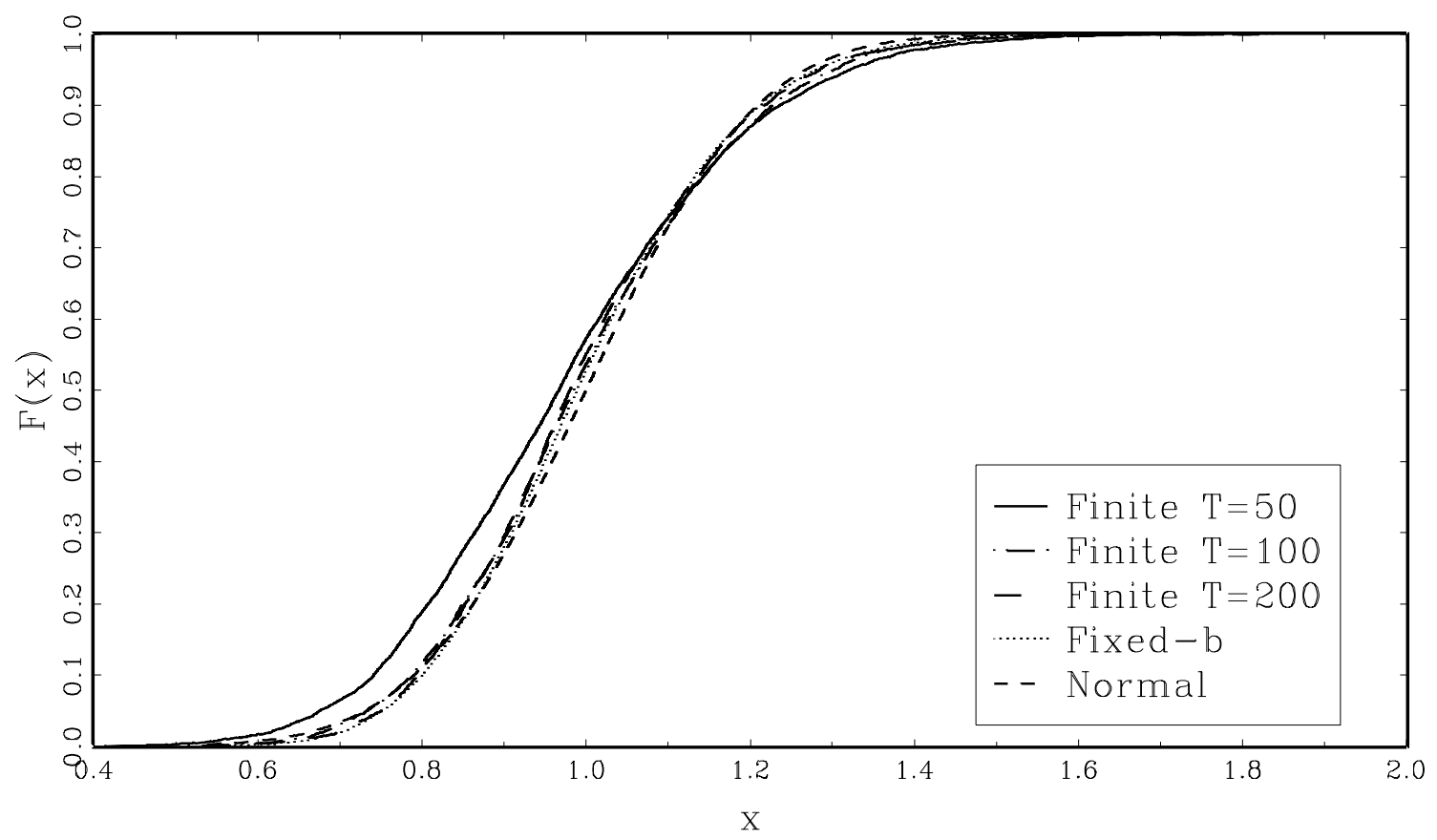

Figure 1.10: CDF Functions, Bartlett Kernel, $\omega=0.5 \pi, \rho=0.0, \quad b=0.04$ 


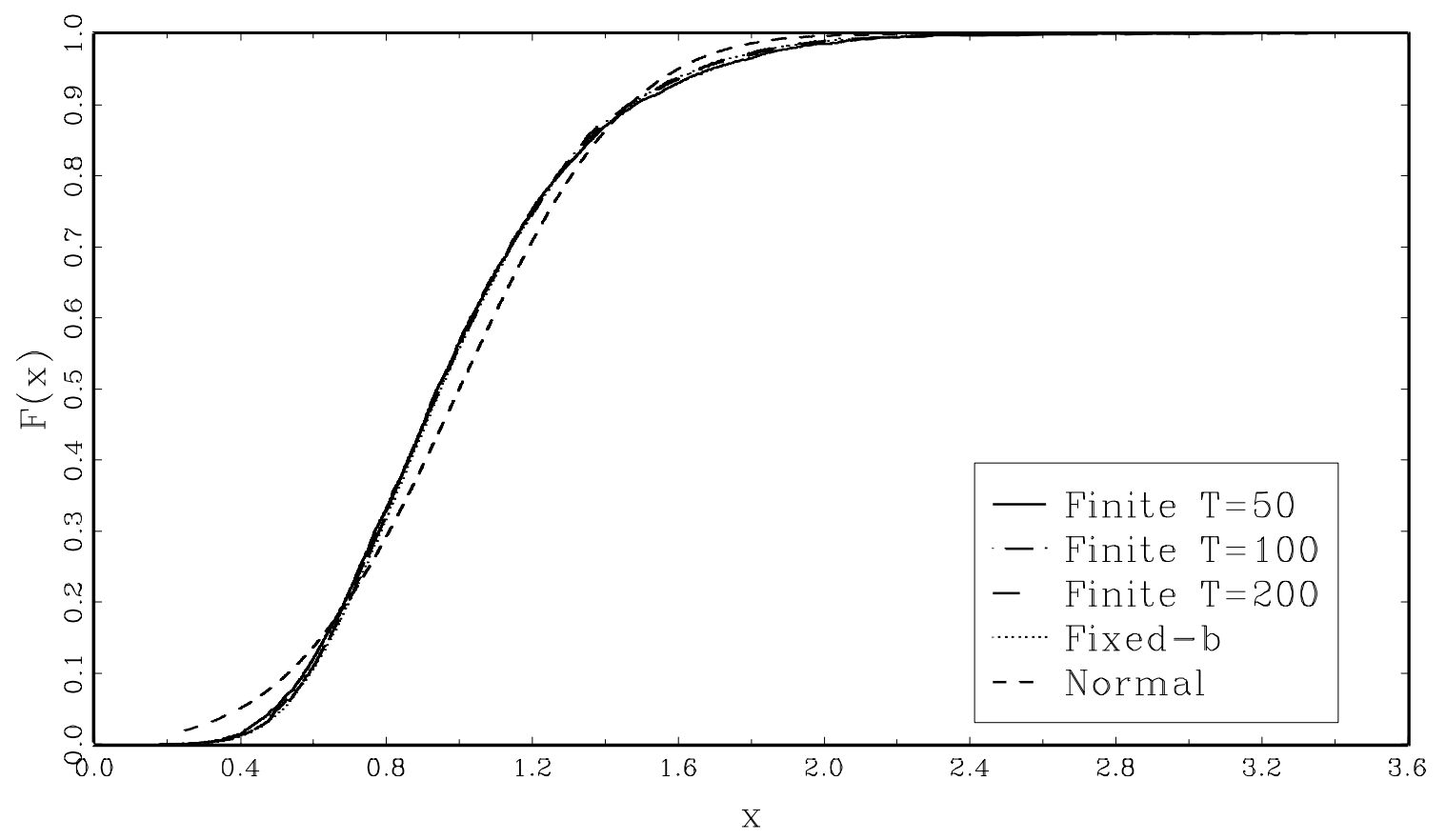

Figure 1.11: CDF Functions, Bartlett Kernel, $\omega=0.5 \pi, \rho=0.0, \quad b=0.2$

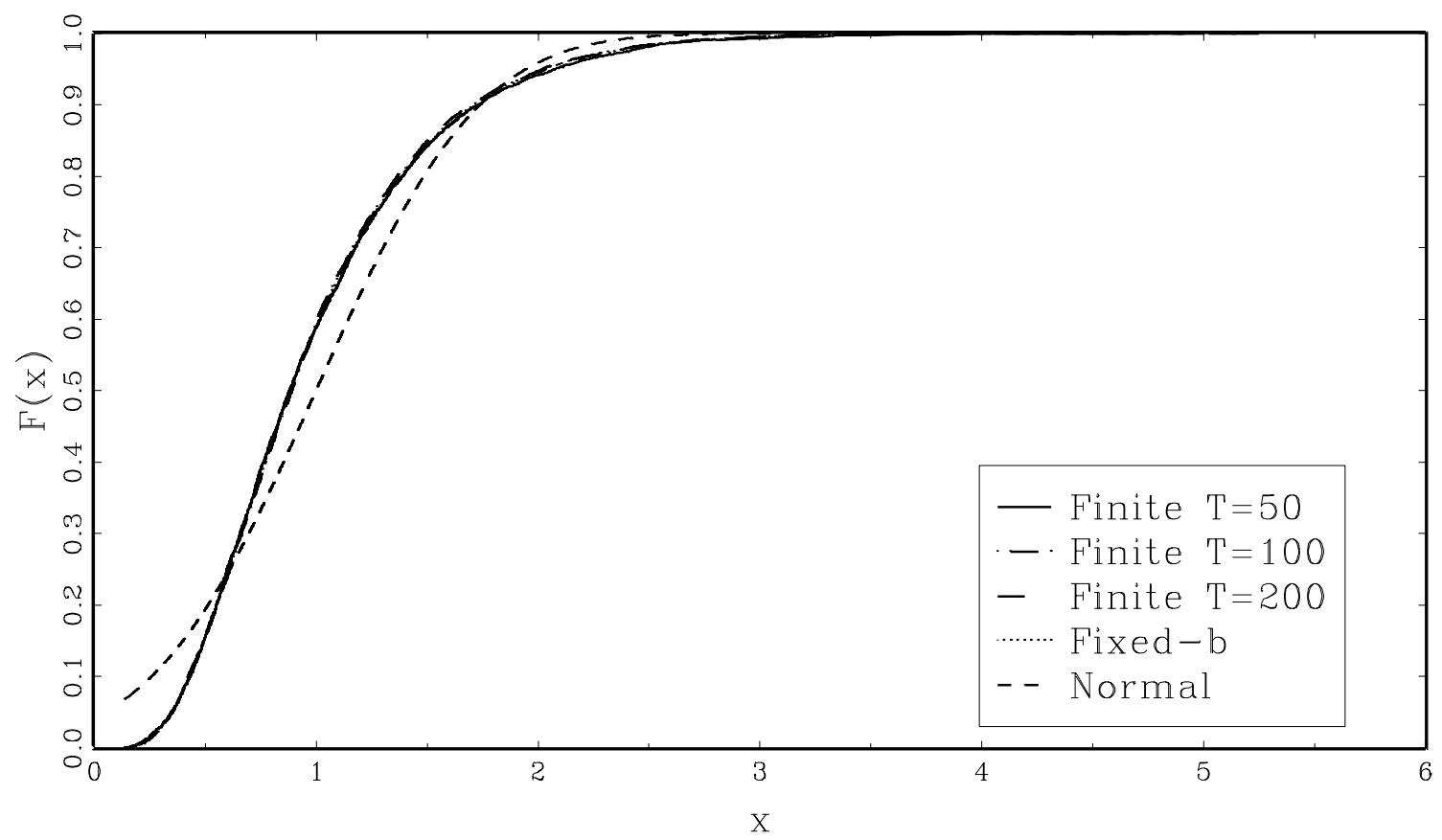

Figure 1.12: CDF Functions, Bartlett Kernel, $\omega=0.5 \pi, \rho=0.0, \quad b=0.5$ 


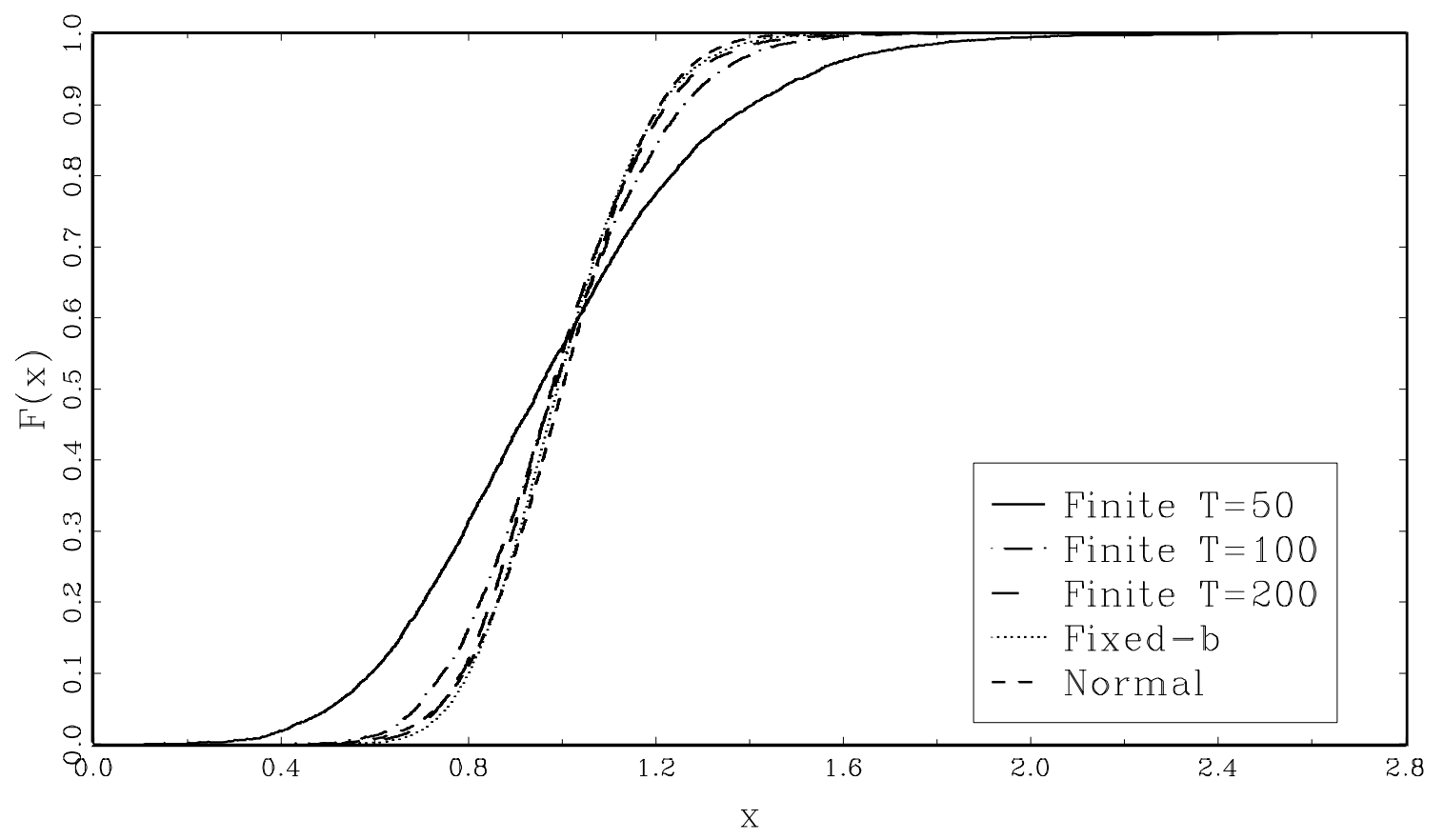

Figure 1.13: CDF Functions, Bartlett Kernel, $\omega=0.5 \pi, \rho=-0.4, \quad b=0.04$

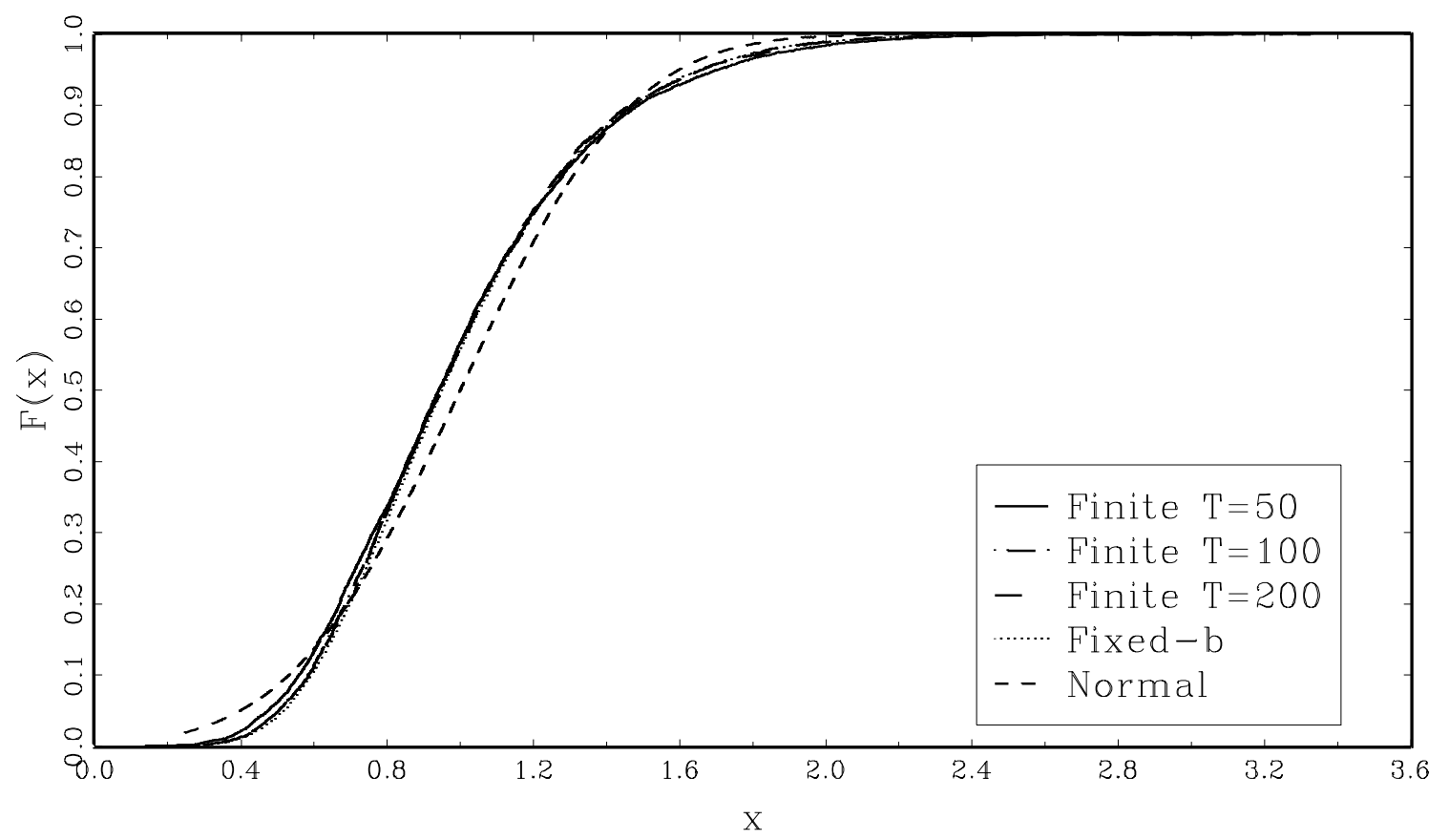

Figure 1.14: CDF Functions, Bartlett Kernel, $\omega=0.5 \pi, \rho=-0.4, \quad b=0.2$ 


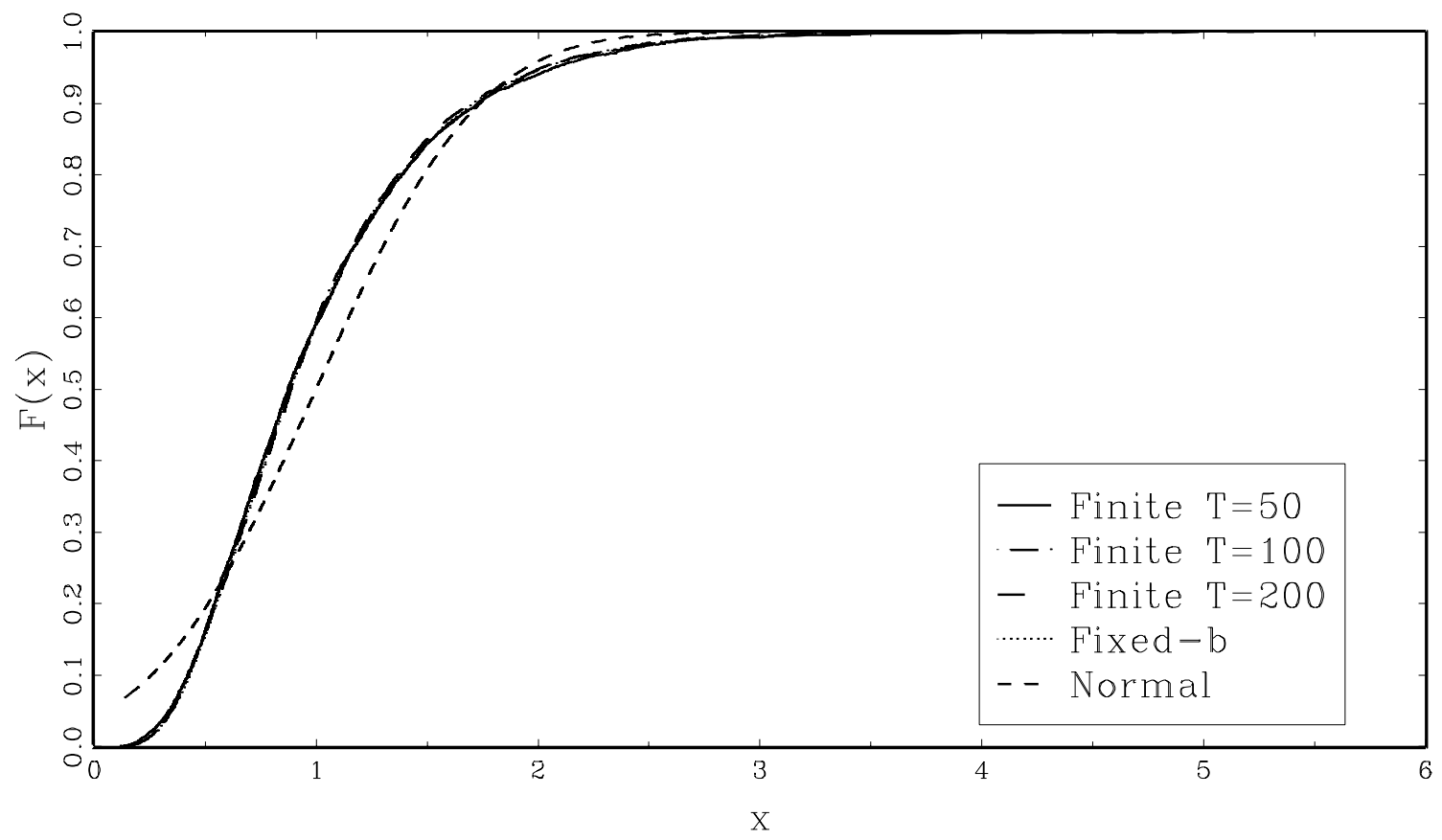

Figure 1.15: CDF Functions, Bartlett Kernel, $\omega=0.5 \pi, \rho=-0.4, \quad b=0.5$

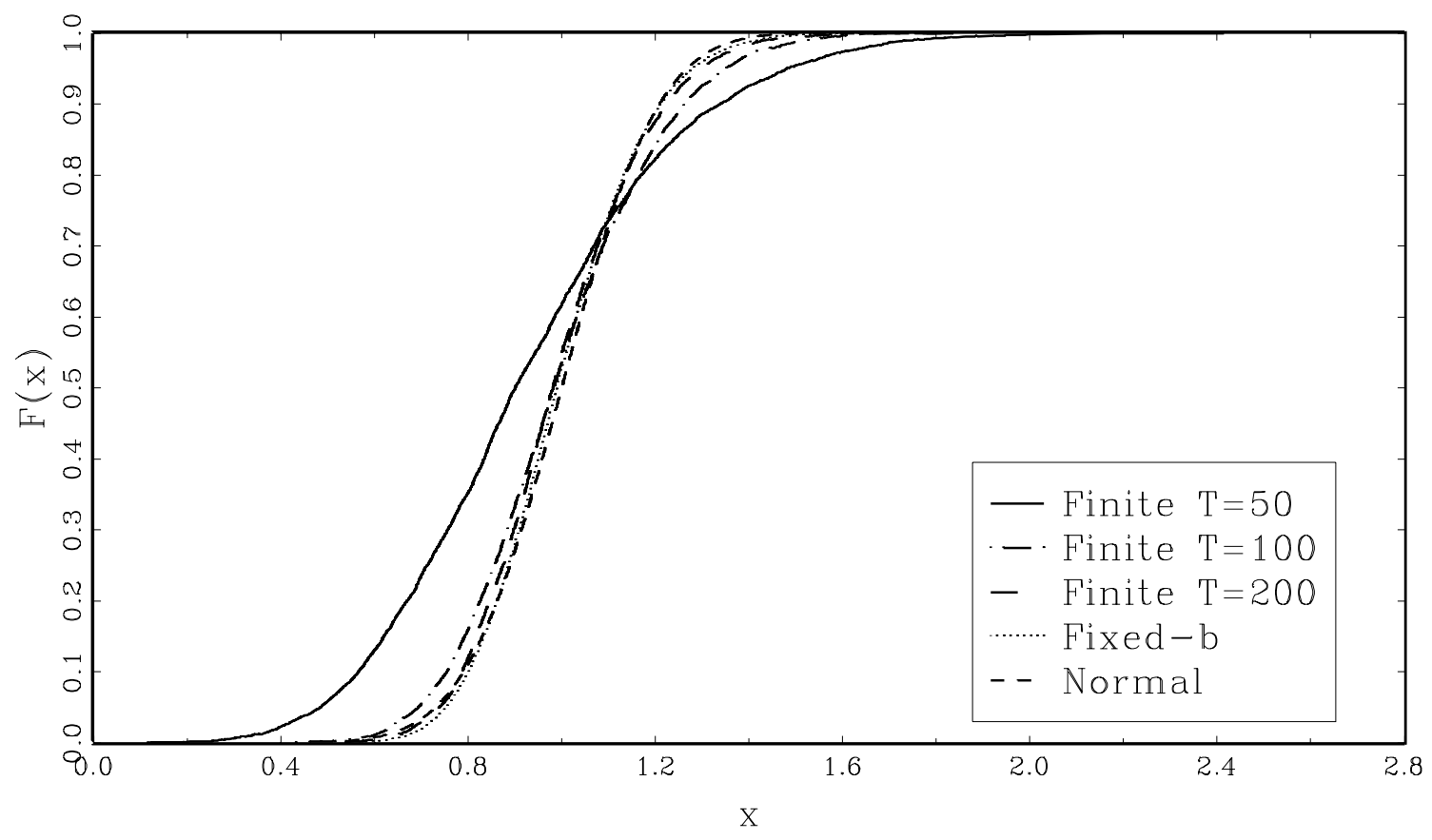

Figure 1.16: CDF Functions, Bartlett Kernel, $\omega=0.5 \pi, \quad \rho=0.4, \quad b=0.04$ 


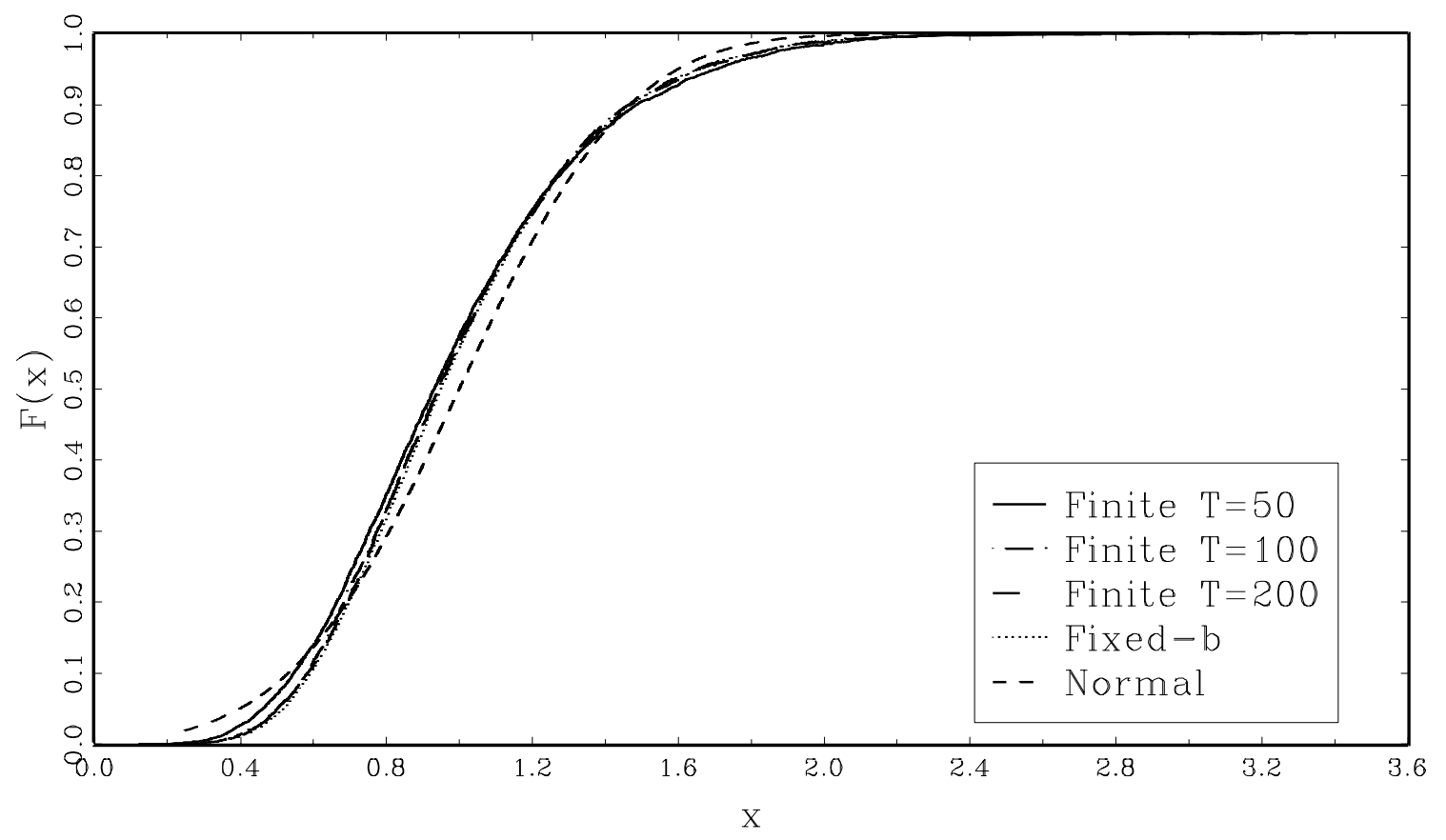

Figure 1.17: CDF Functions, Bartlett Kernel, $\omega=0.5 \pi, \rho=-0.4, \quad b=0.2$

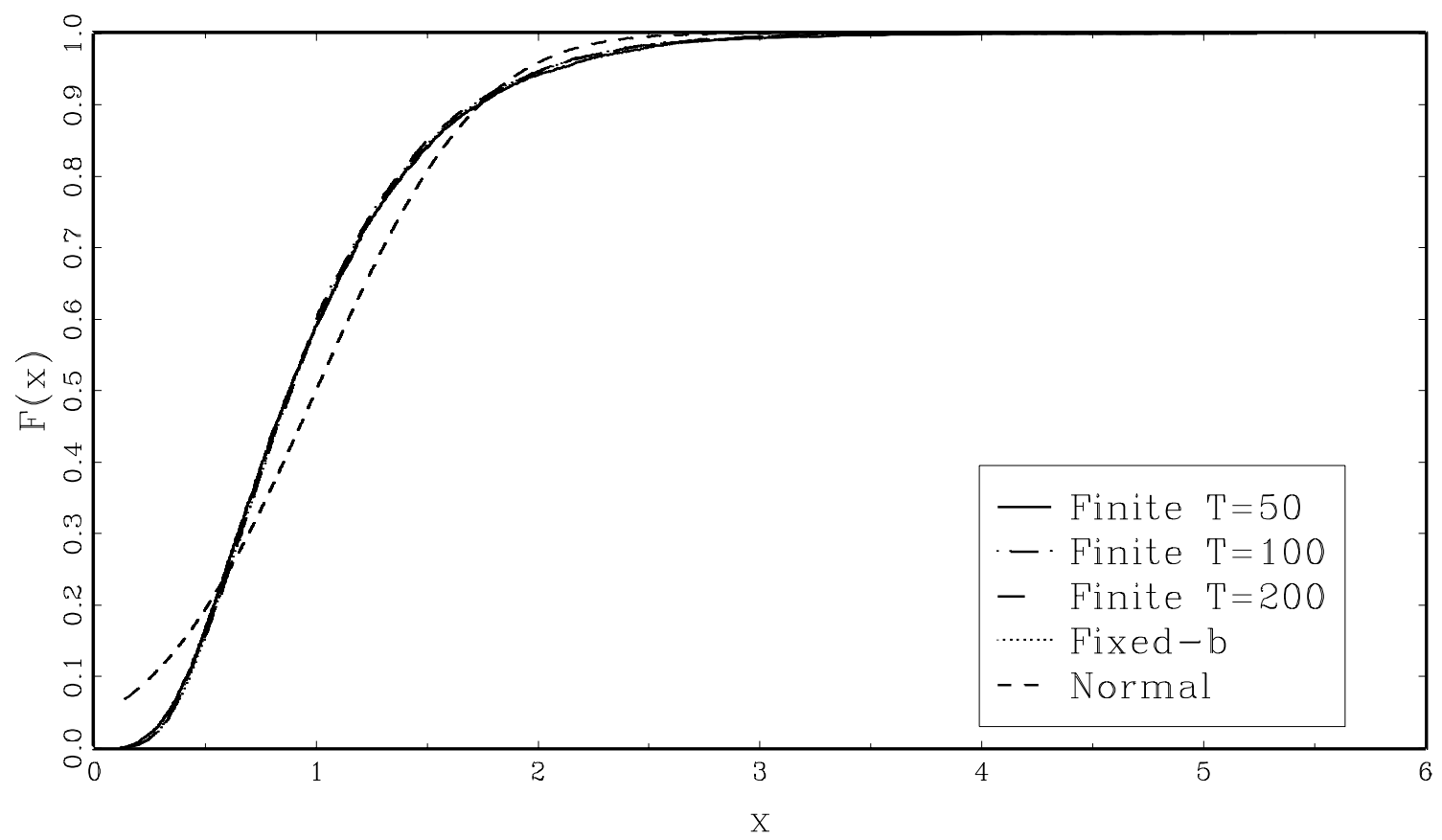

Figure 1.18: CDF Functions, Bartlett Kernel, $\omega=0.5 \pi, \rho=0.4, \quad b=0.5$ 


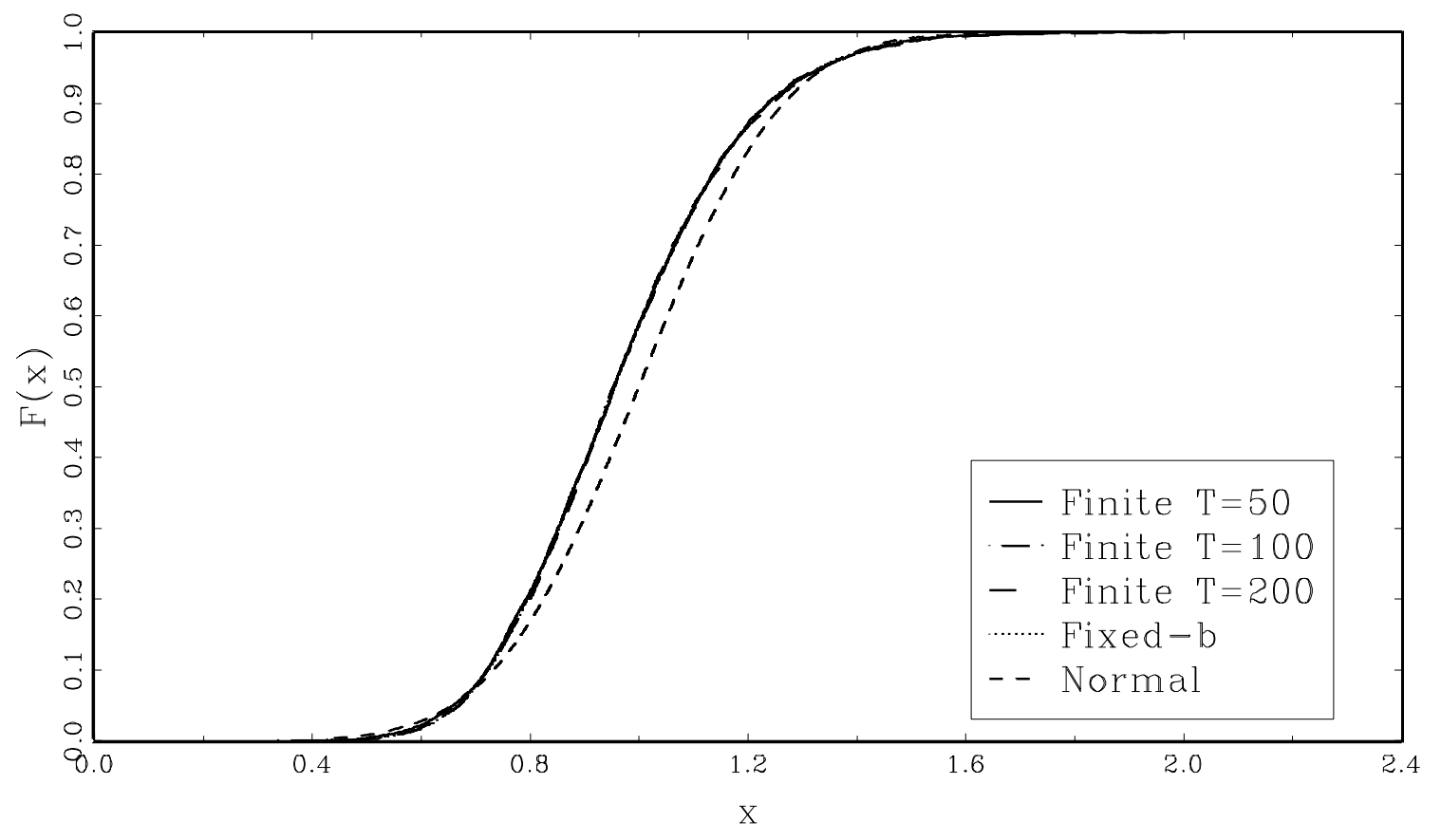

Figure 2.1: CDF Functions, Parzen Kernel, $\omega=0, \quad \rho=0, \quad b=0.04$

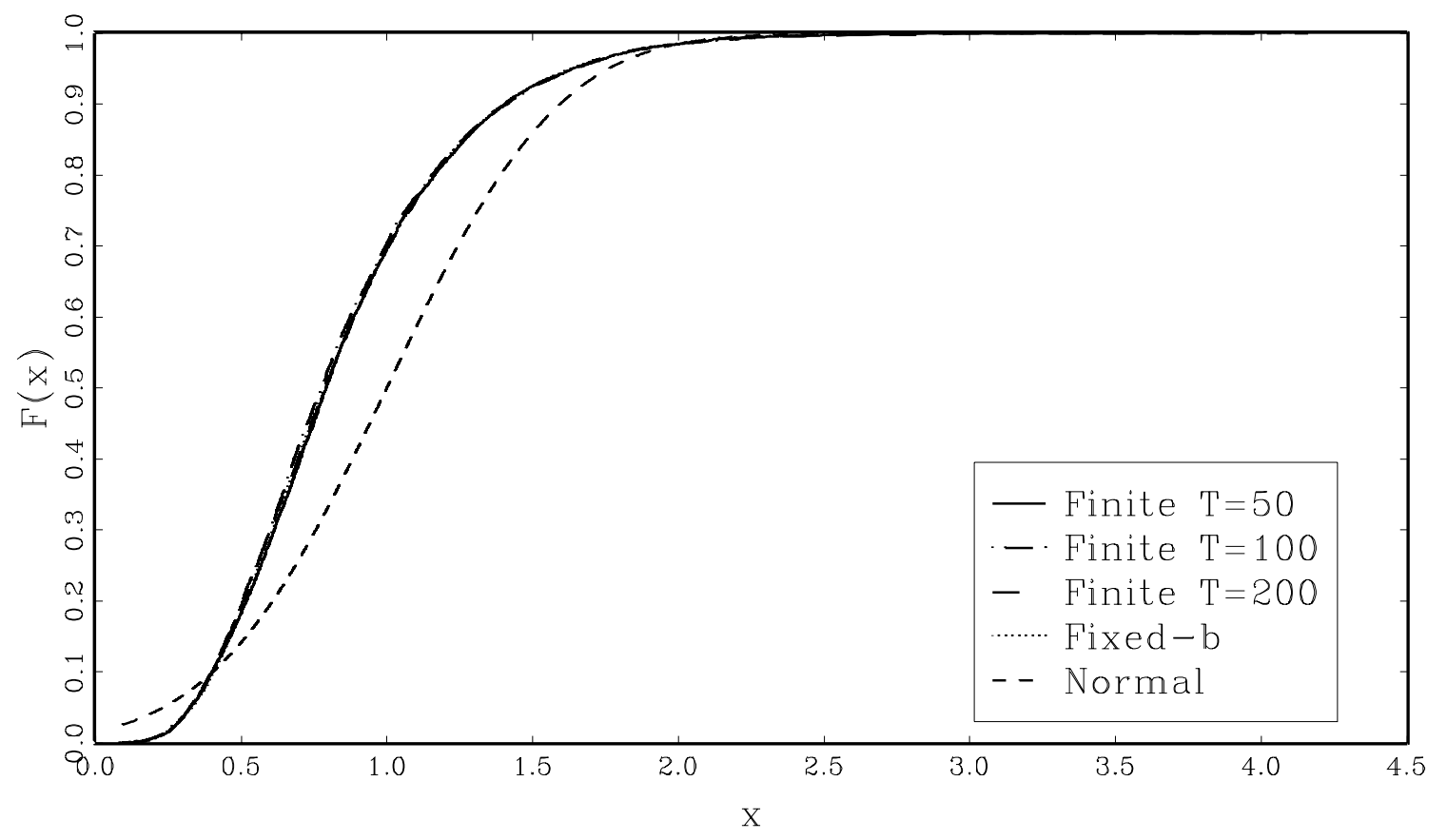

Figure 2.2: CDF Functions, Parzen Kernel, $\omega=0, \rho=0, \quad b=0.2$ 


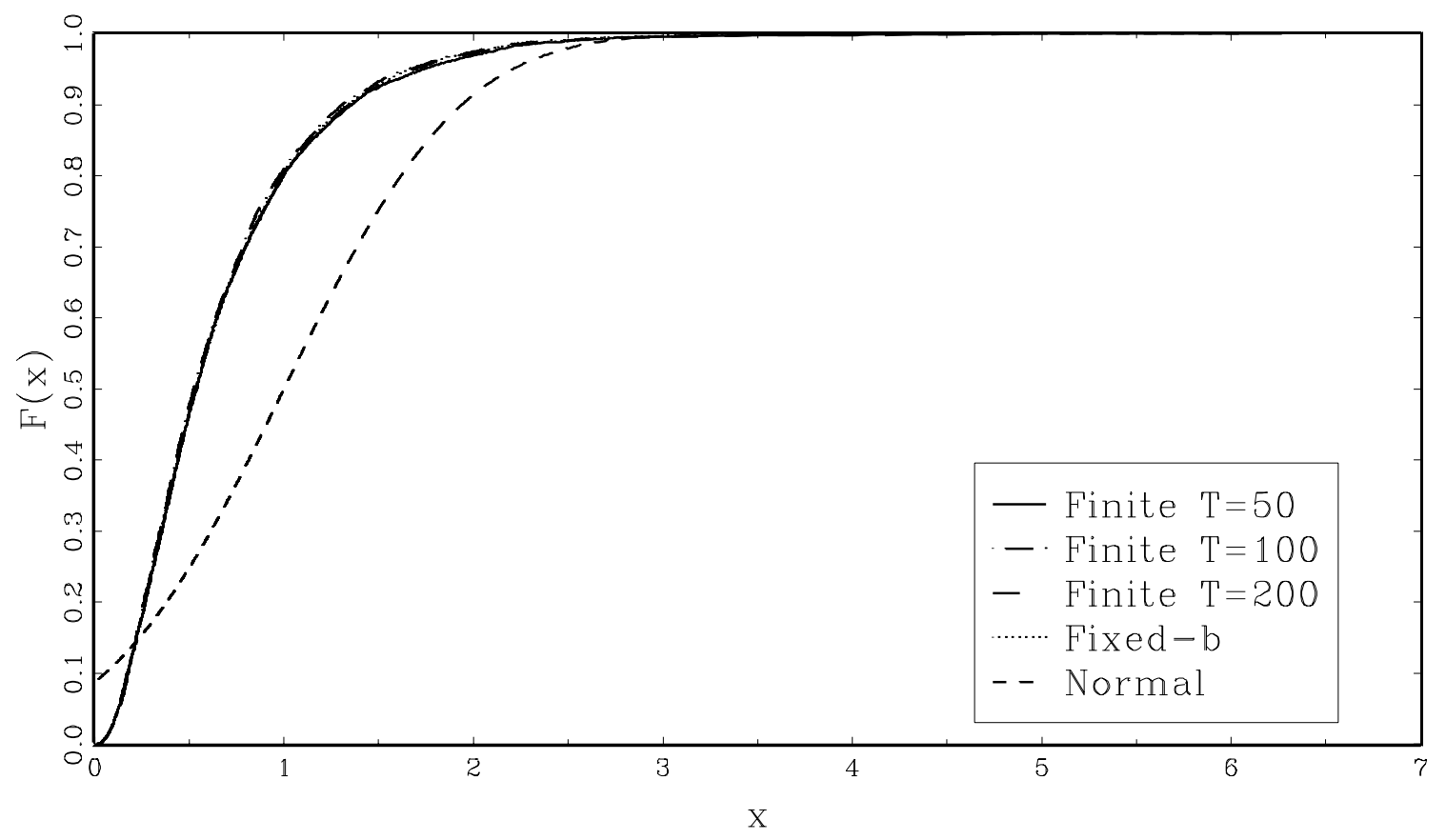

Figure 2.3: CDF Functions, Parzen Kernel, $\omega=0, p=0, \quad b=0.5$

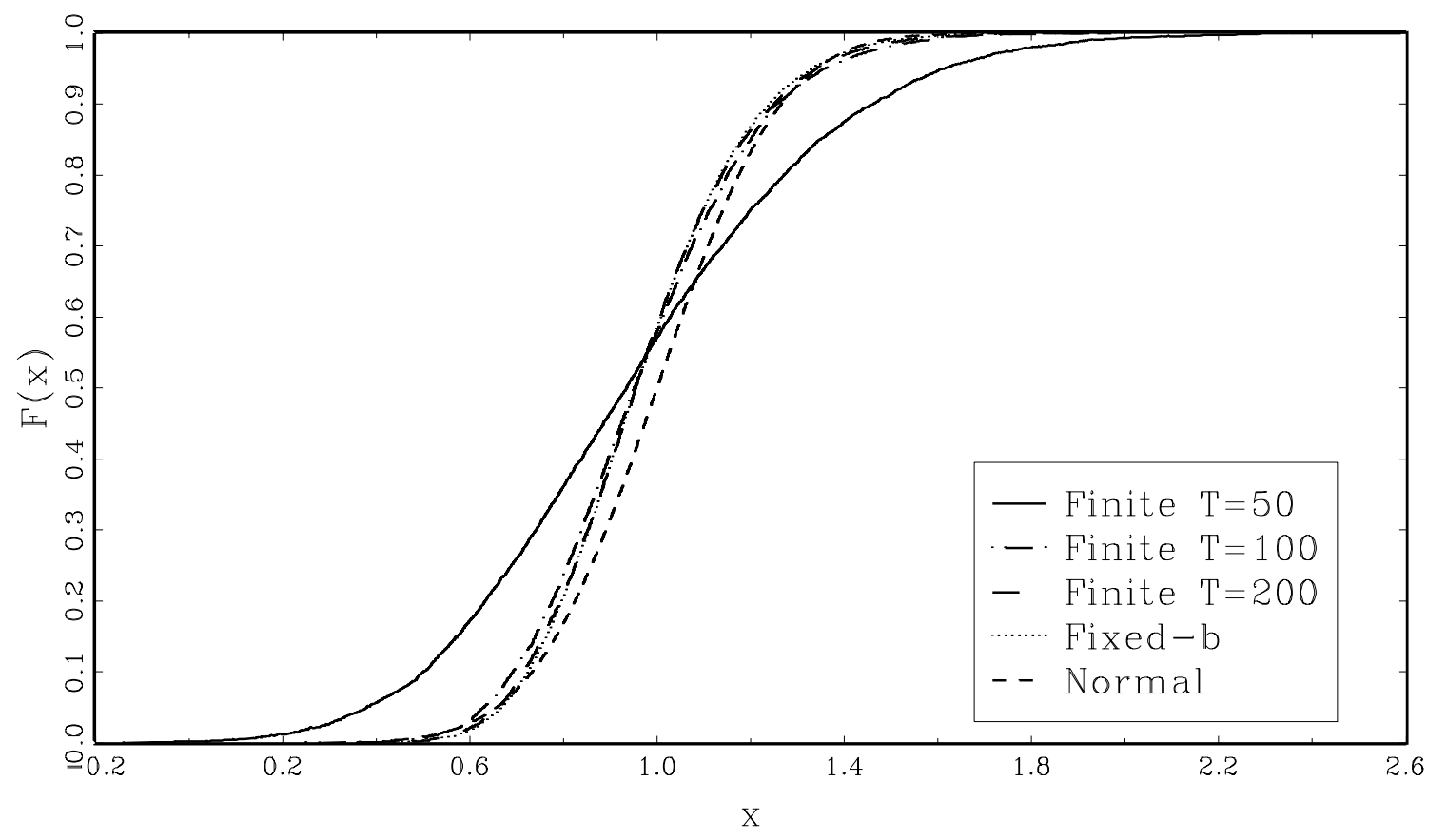

Figure 2.4: CDF Functions, Parzen Kernel, $\omega=0, p=-0.4, \quad b=0.04$ 


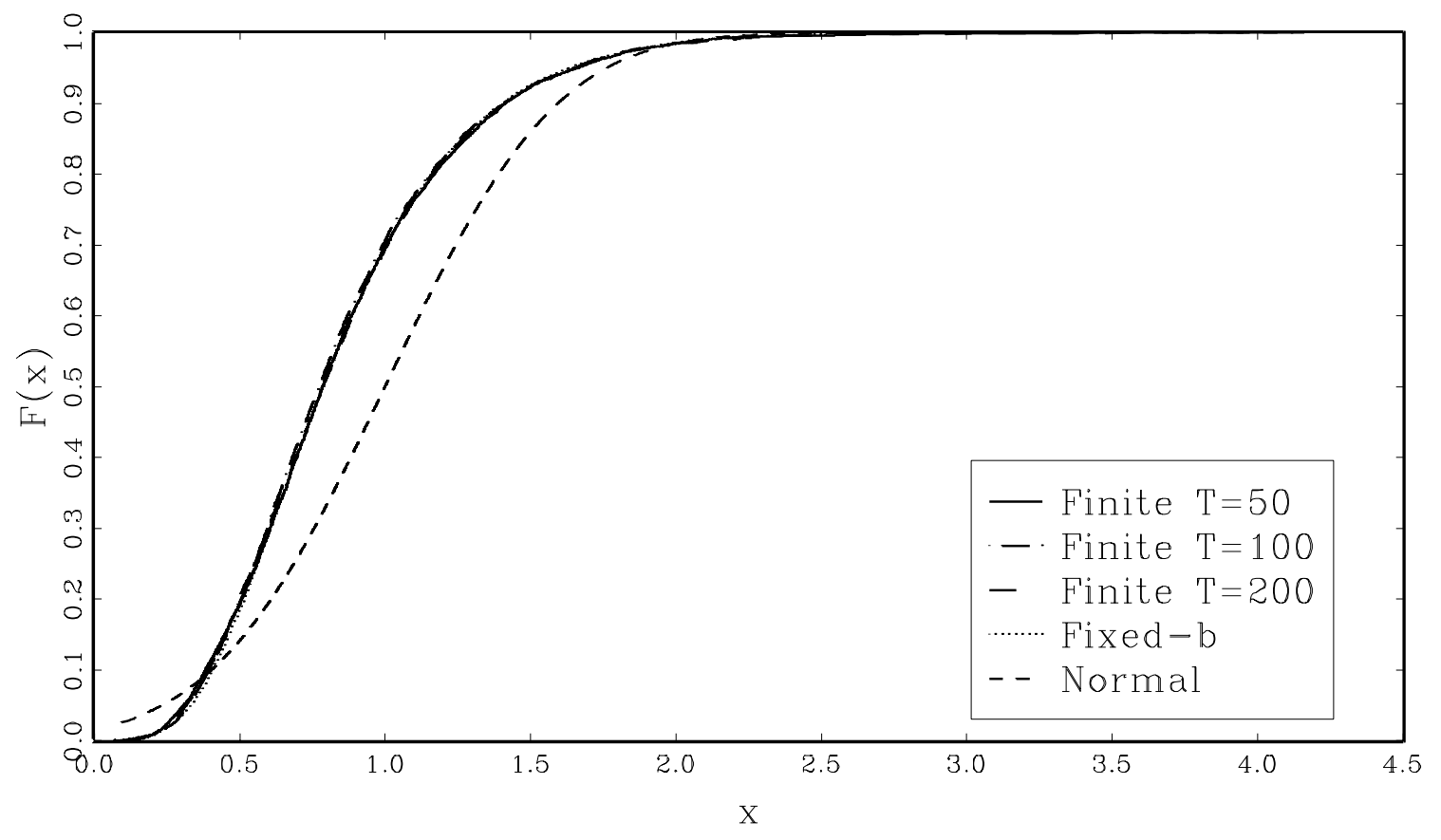

Figure 2.5: CDF Functions, Parzen Kernel, $\omega=0, p=-0.4, \quad b=0.2$

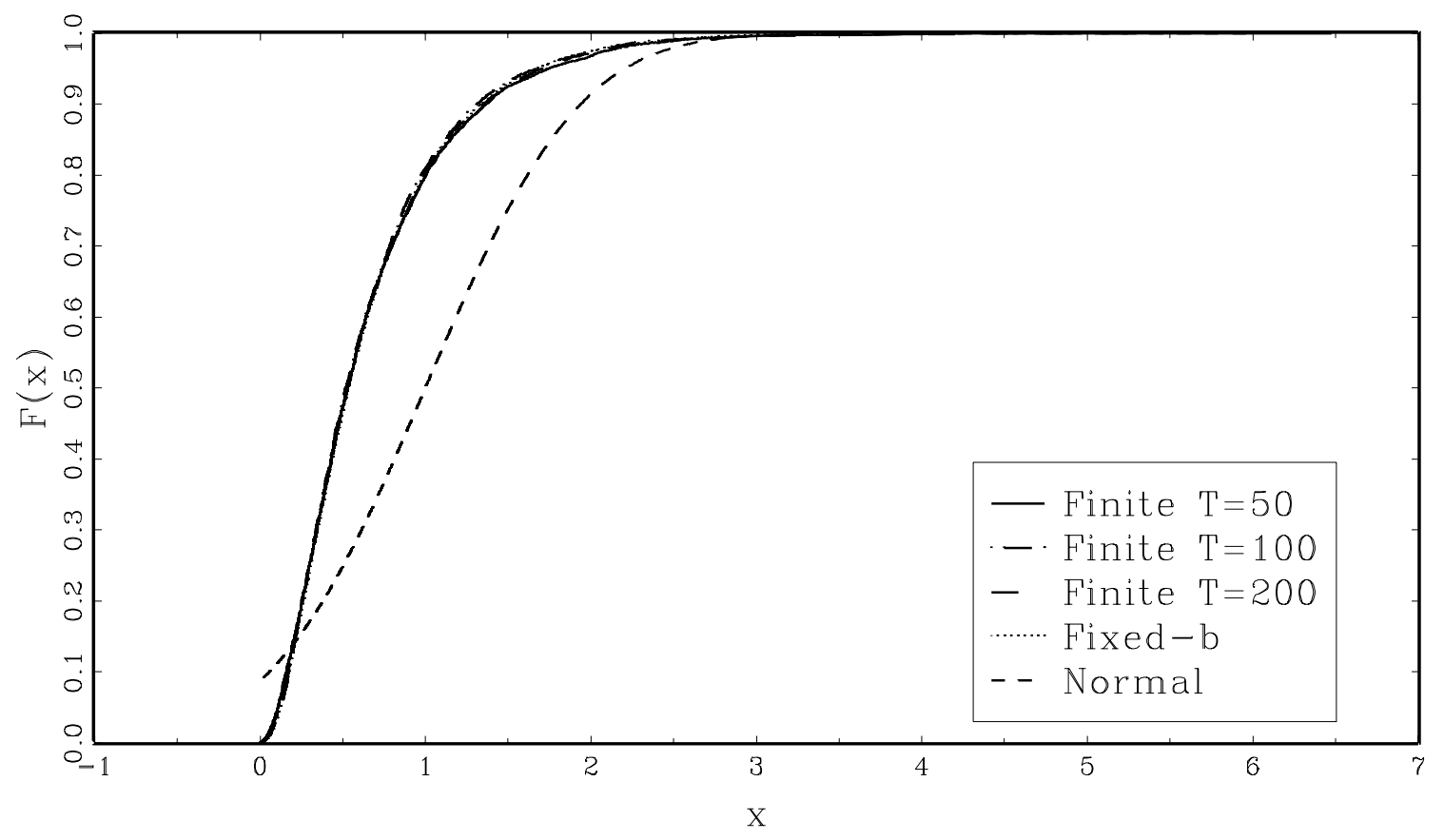

Figure 2.6: CDF Functions, Parzen Kernel, $\omega=0, p=-0.4, b=0.5$ 


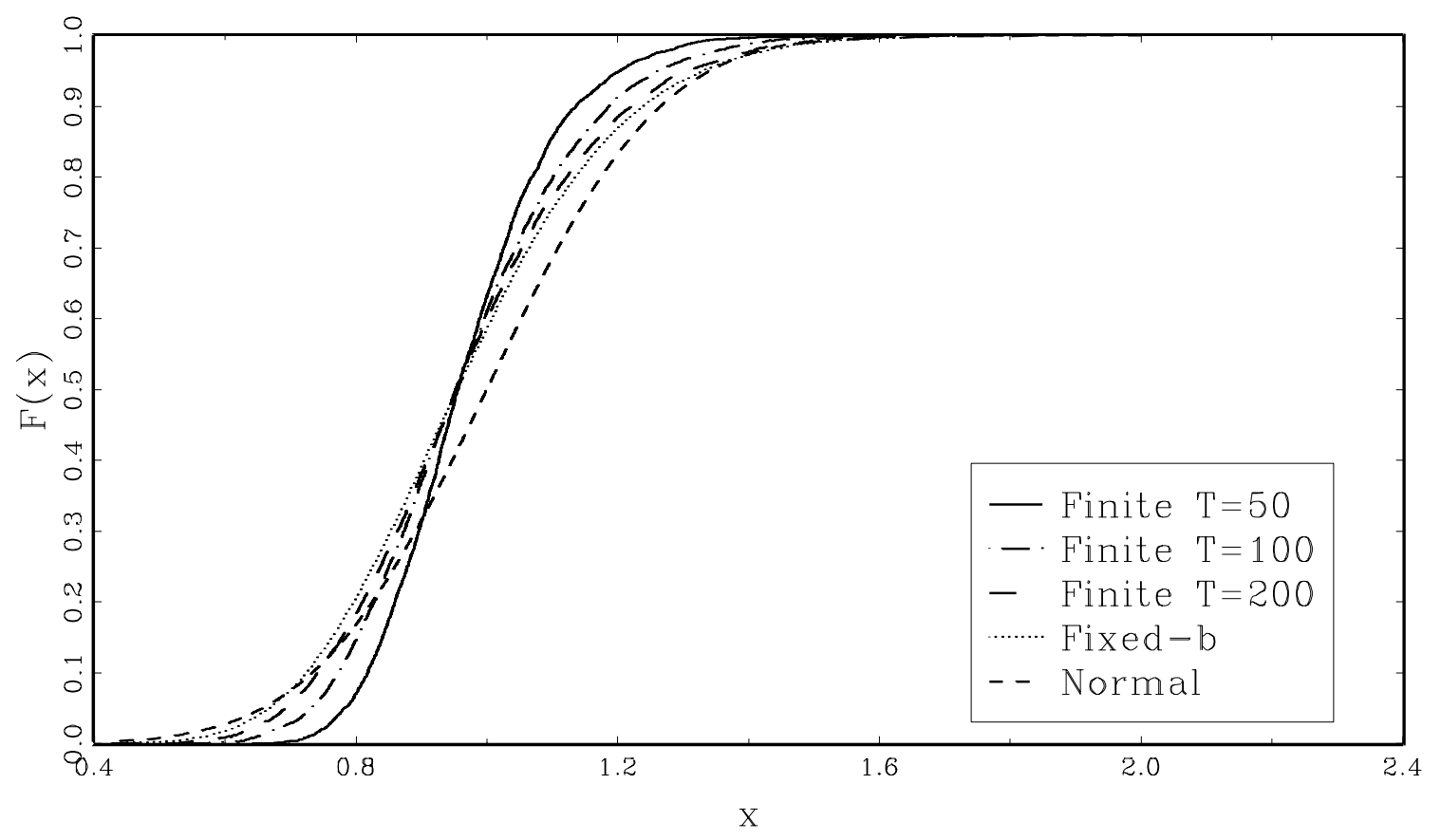

Figure 2.7: CDF Functions, Parzen Kernel, $\omega=0, \rho=0.4, b=0.04$

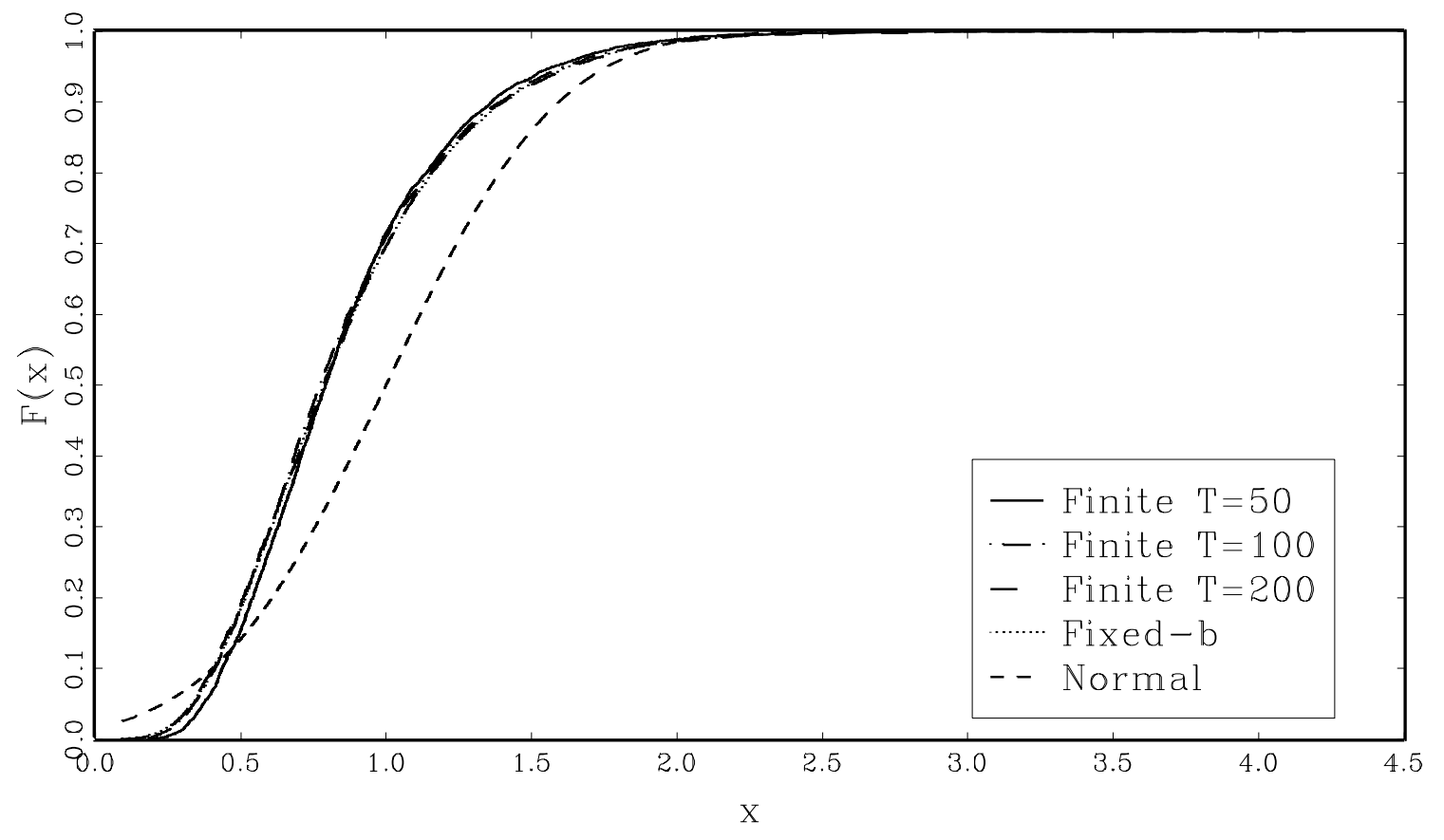

Figure 2.8: CDF Functions, Parzen Kernel, $\omega=0, \rho=0.4, \quad b=0.2$ 


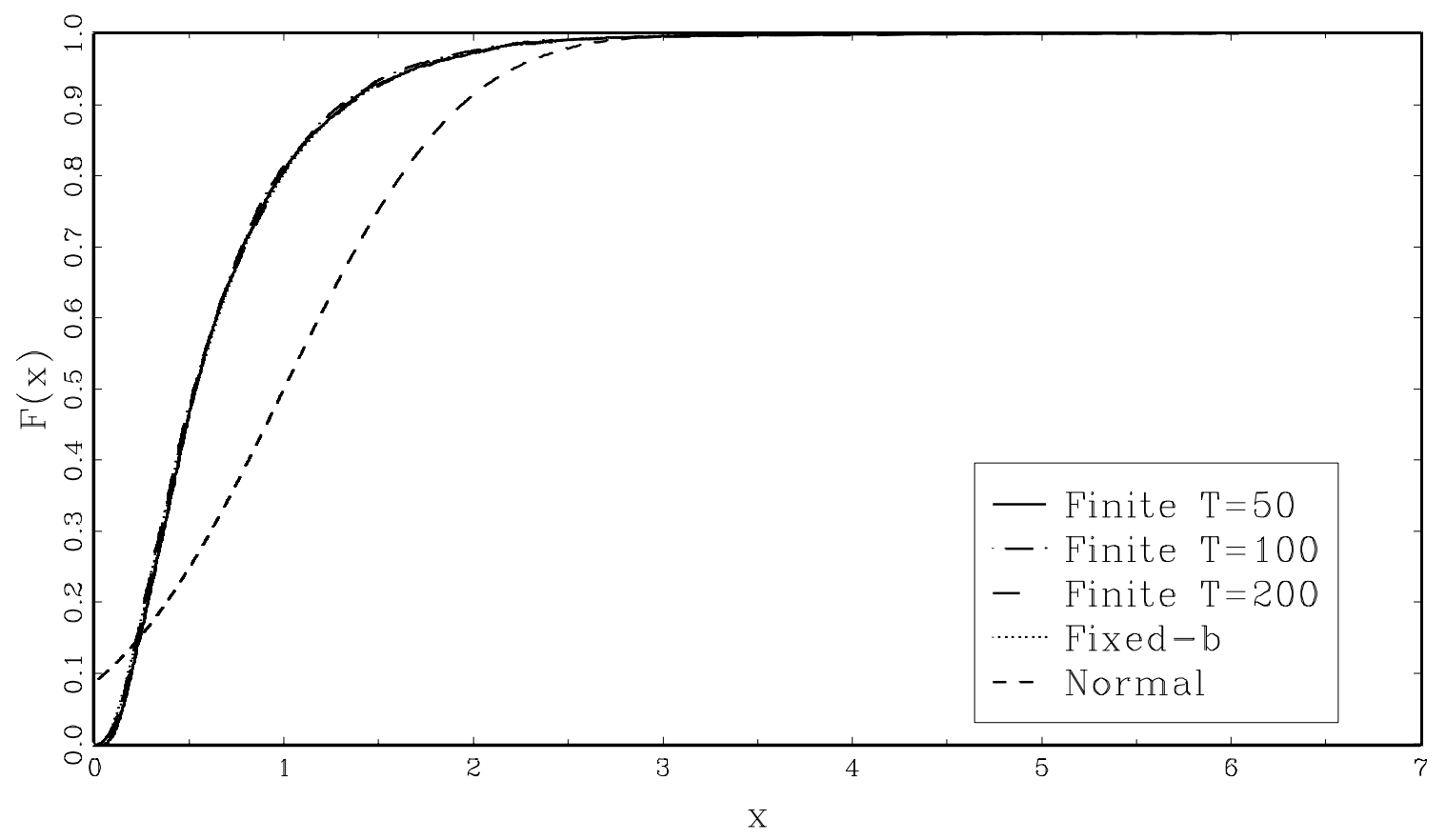

Figure 2.9: CDF Functions, Parzen Kernel, $\omega=0, \rho=0.4, b=0.5$

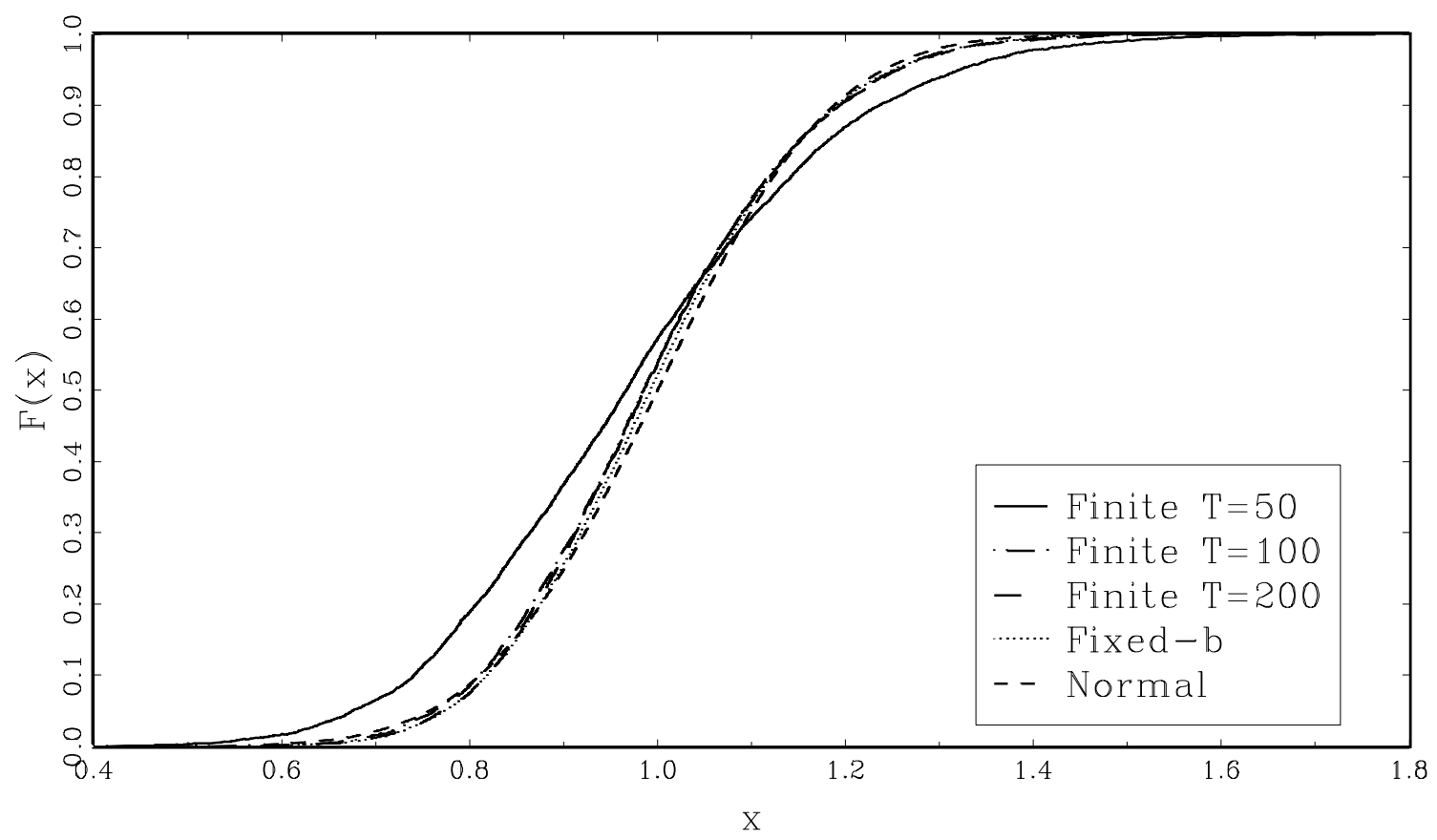

Figure 2.10: CDF Functions, Parzen Kernel, $\omega=0.5 \pi, \rho=0.0, b=0.04$ 


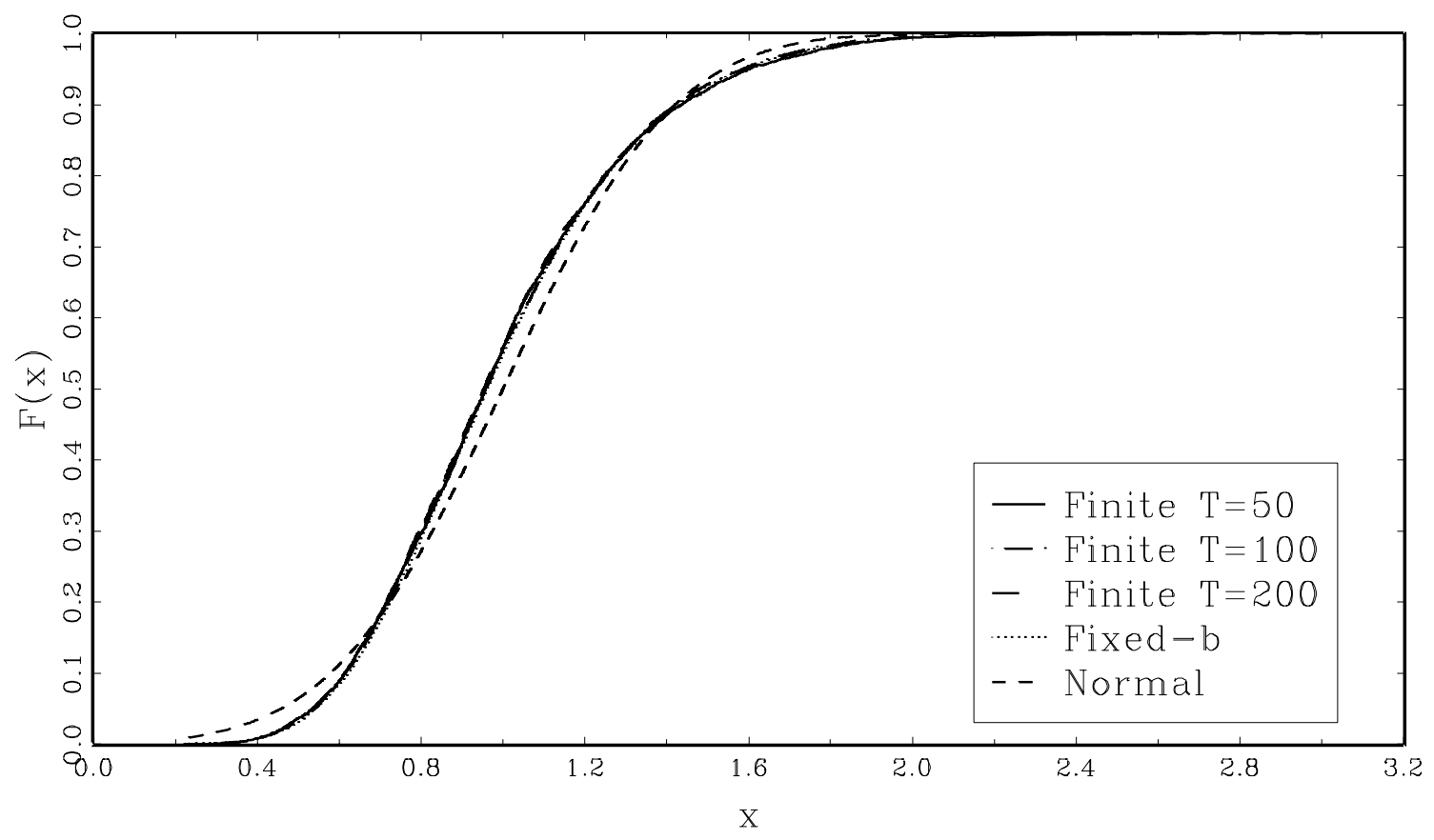

Figure 2.11: CDF Functions, Parzen Kernel, $\omega=0.5 \pi, \quad \rho=0.0, \quad b=0.2$

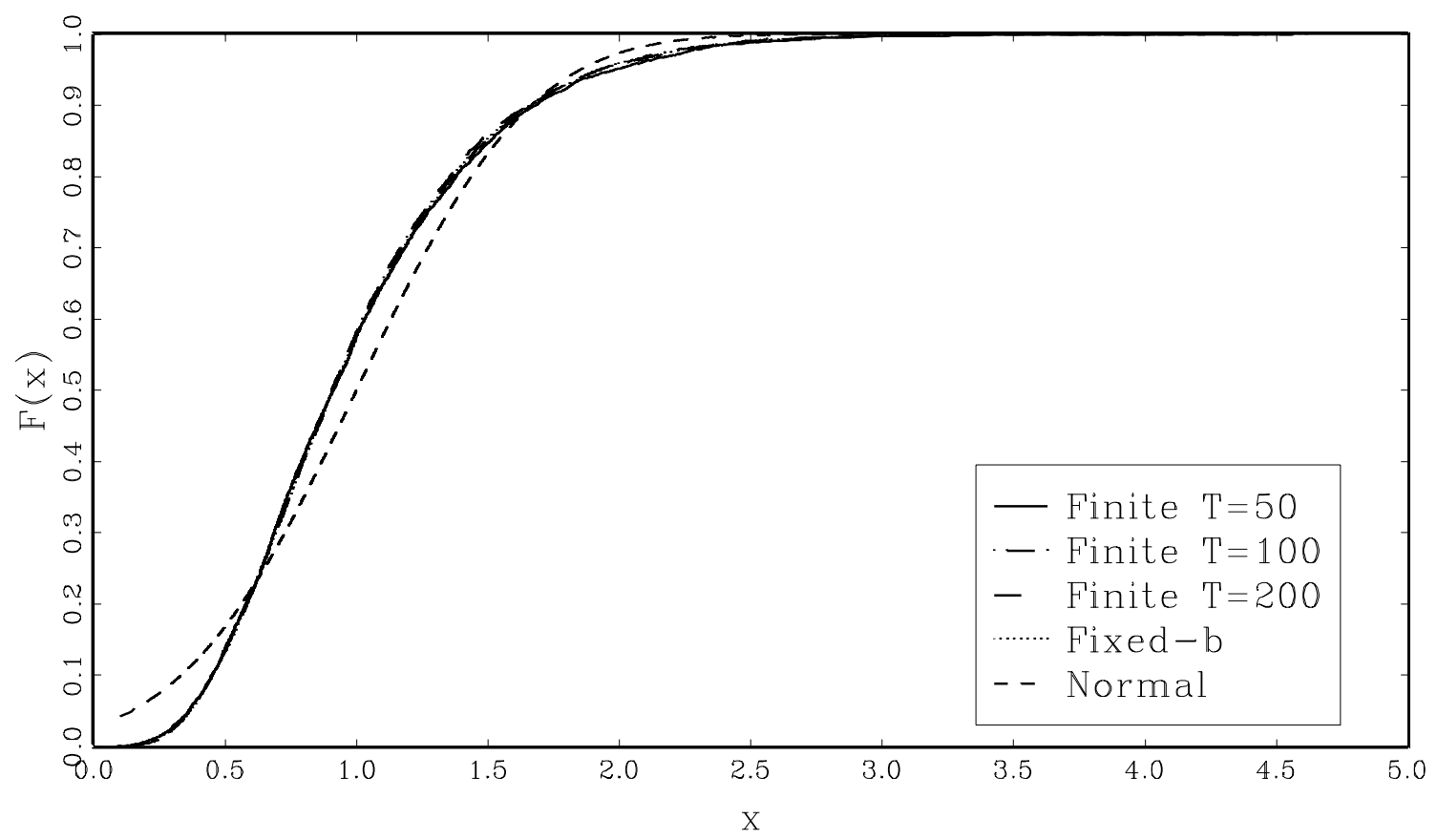

Figure 2.12: CDF Functions, Parzen Kernel, $\omega=0.5 \pi, \quad \rho=0.0, \quad b=0.5$ 


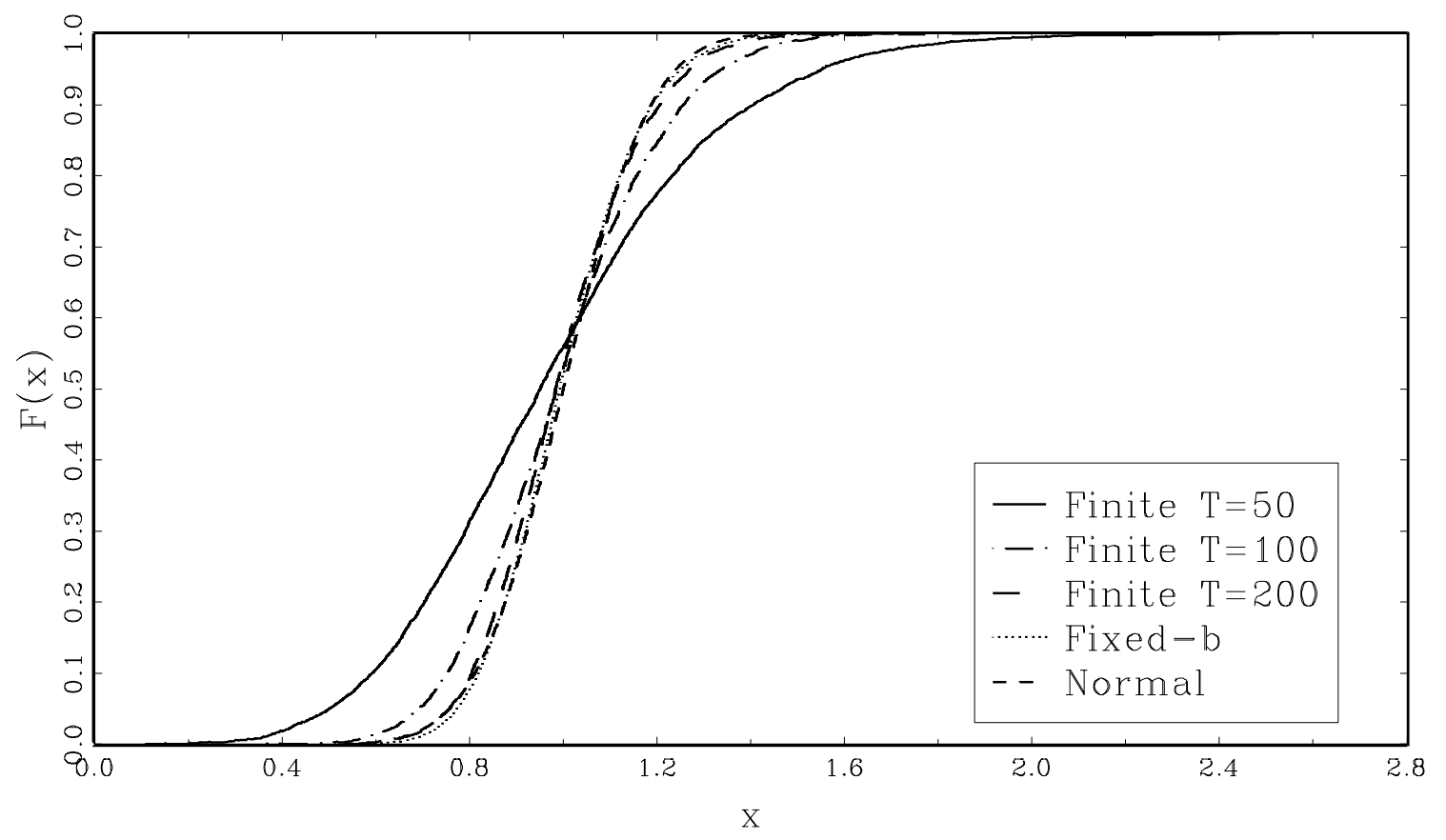

Figure 2.13: CDF Functions, Parzen Kernel, $\omega=0.5 \pi, \rho=-0.4, \quad b=0.04$

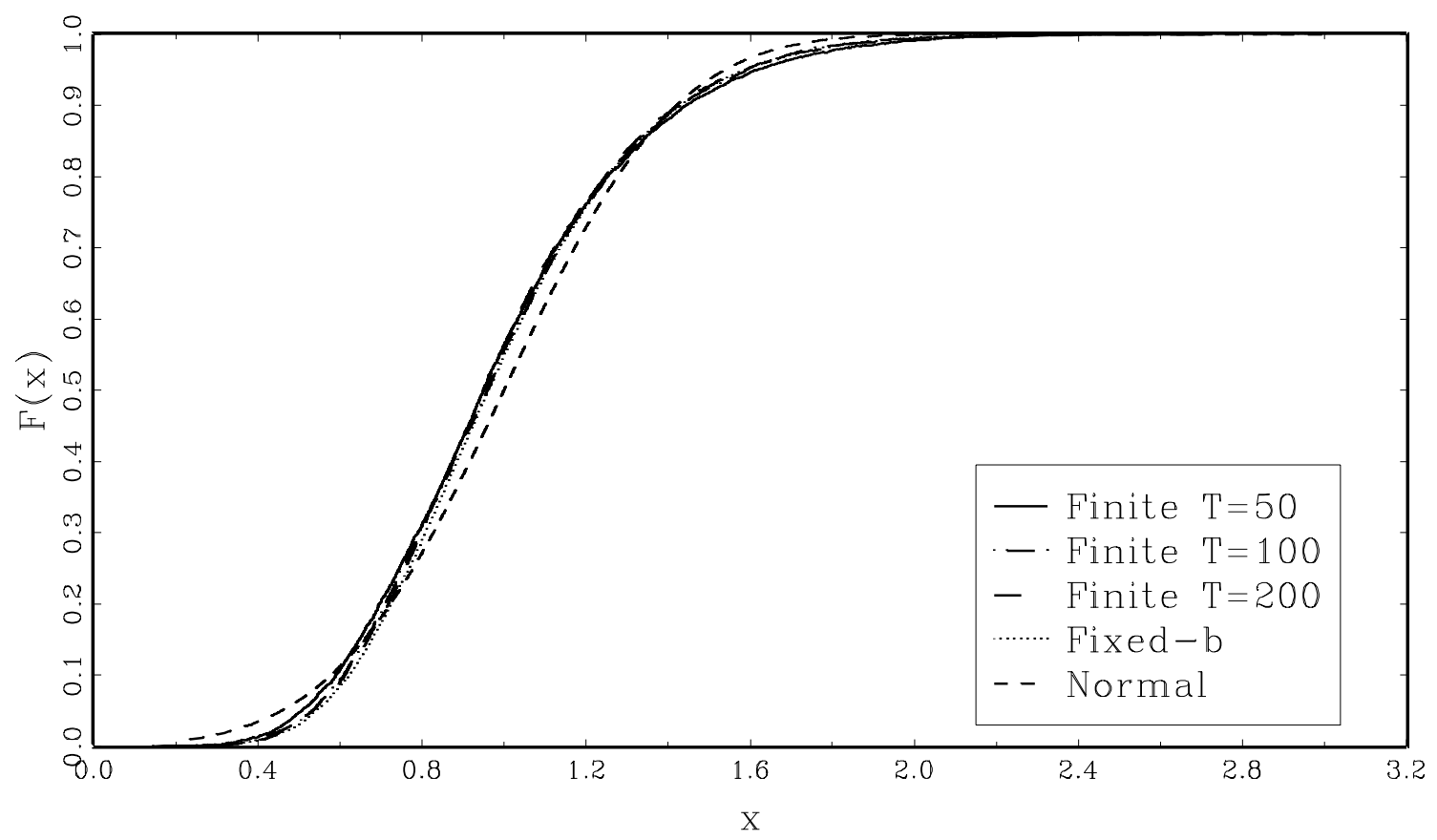

Figure 2.14: CDF Functions, Parzen Kernel, $\omega=0.5 \pi, \rho=-0.4, b=0.2$ 


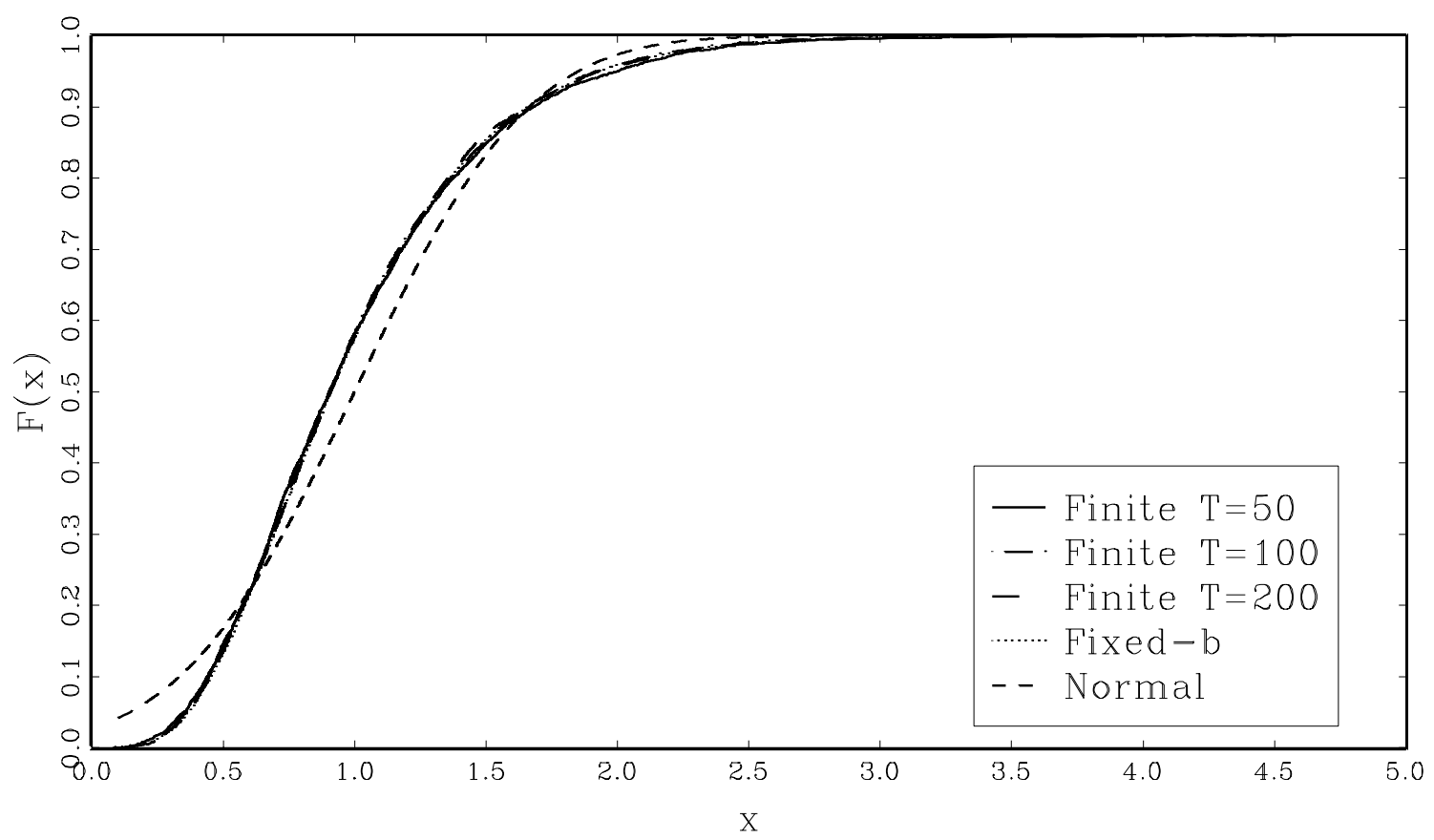

Figure 2.15: CDF Functions, Parzen Kernel, $\omega=0.5 \pi, \rho=-0.4, b=0.5$

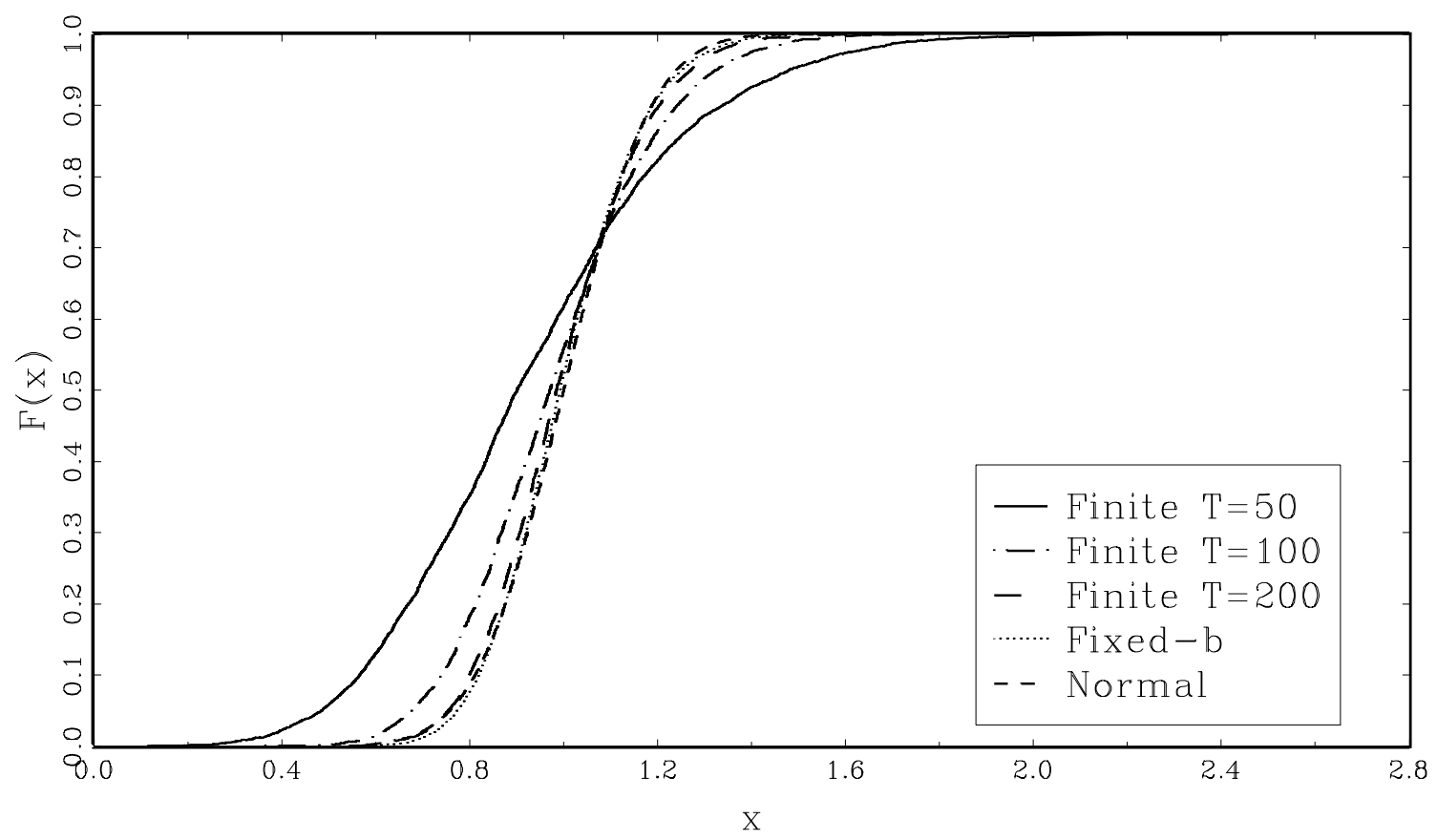

Figure 2.16: CDF Functions, Parzen Kernel, $\omega=0.5 \pi, \rho=0.4, b=0.04$ 


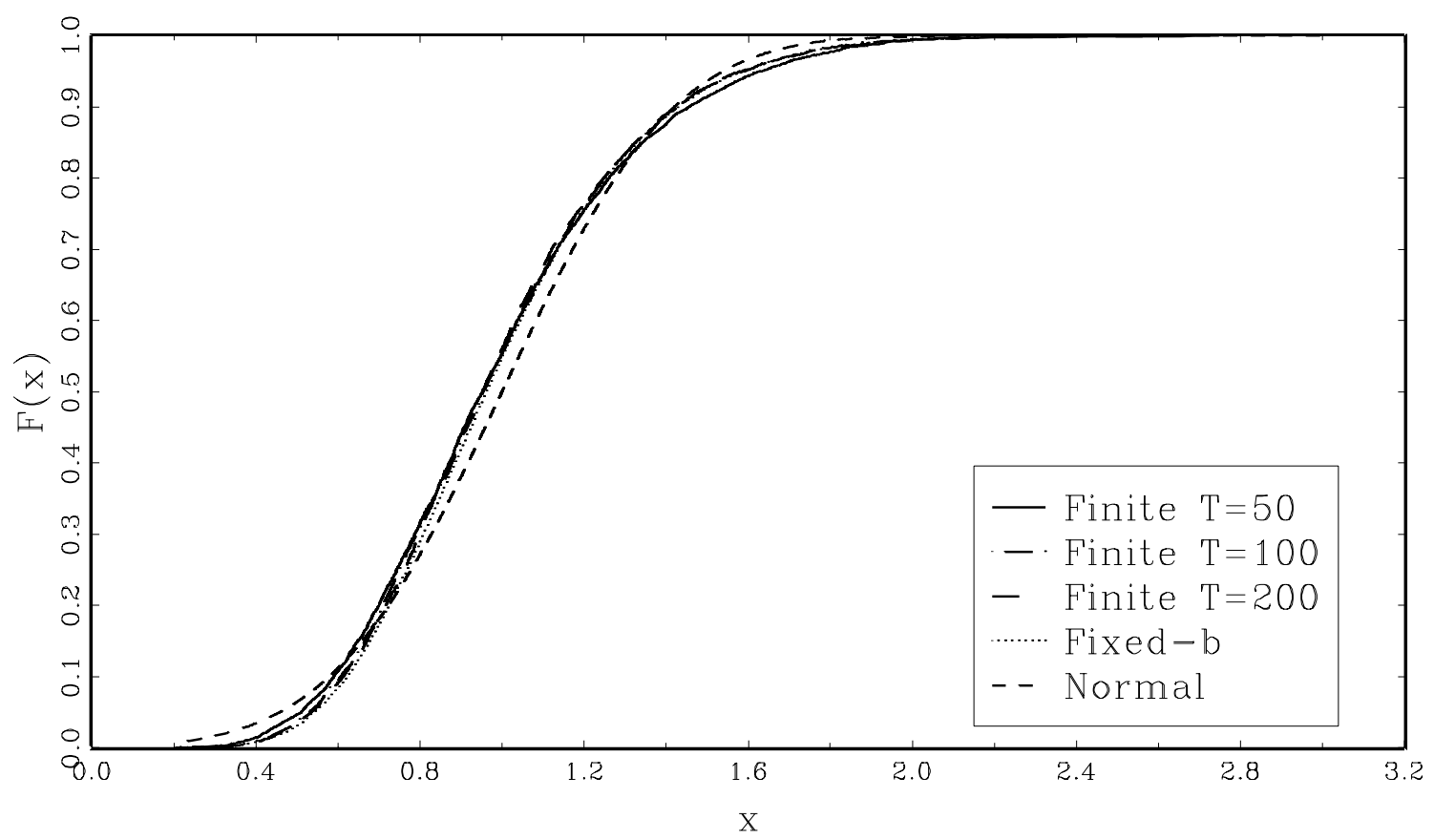

Figure 2.17: CDF Functions, Parzen Kernel, $\omega=0.5 \pi, \rho=-0.4, b=0.2$

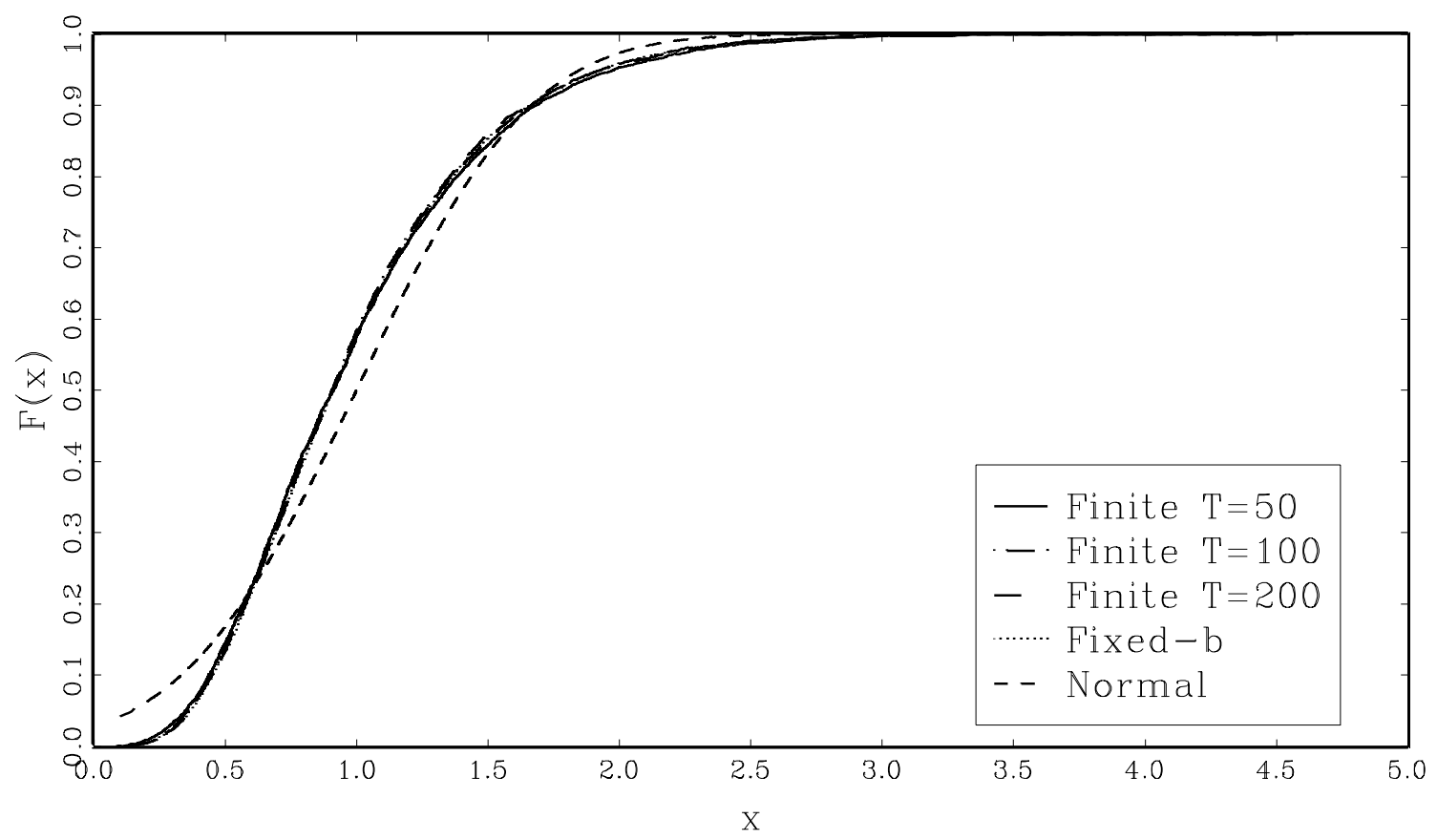

Figure 2.18: CDF Functions, Parzen Kernel, $\omega=0.5 \pi, \rho=0.4, b=0.5$ 


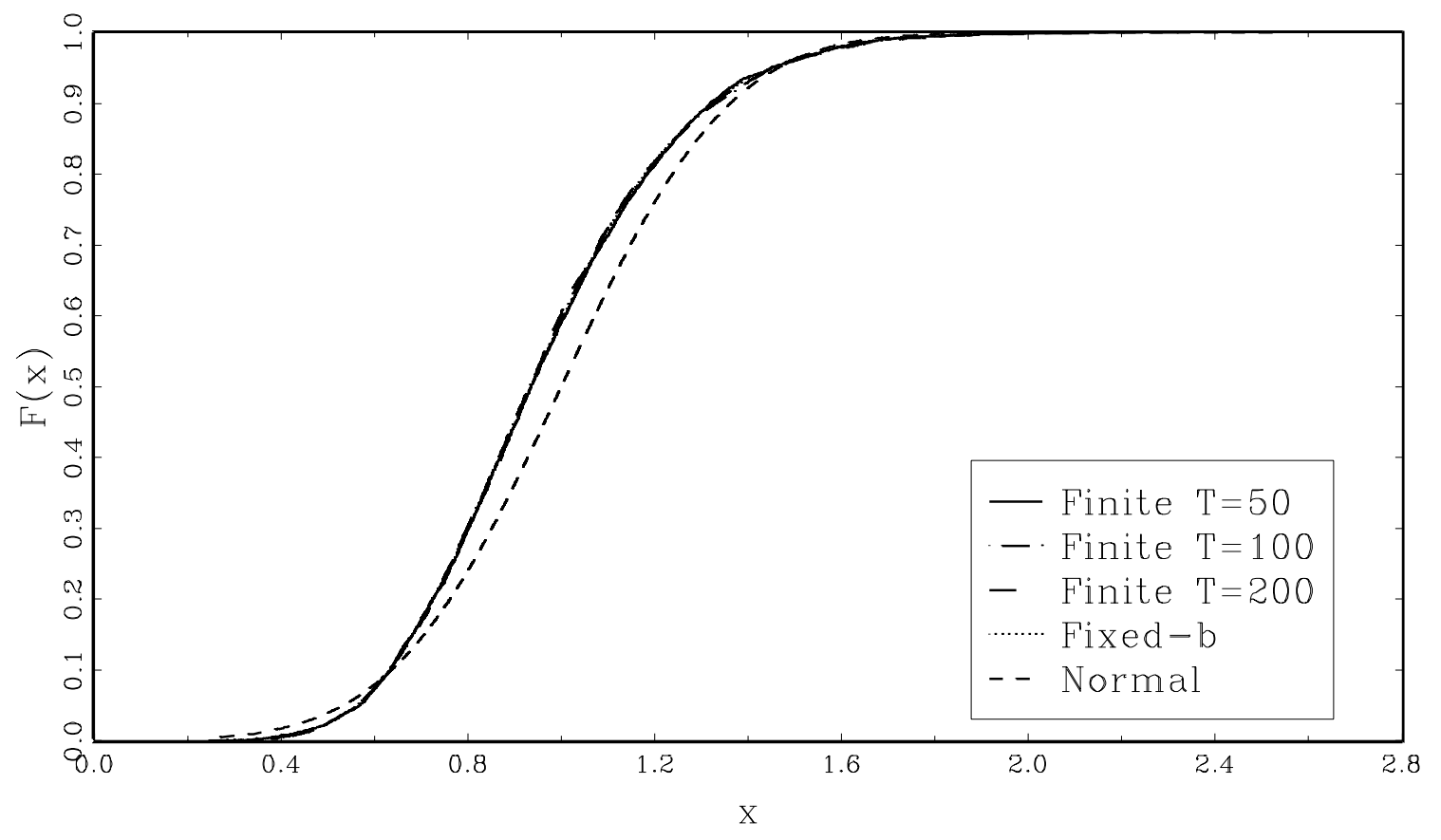

Figure 3.1: CDF Functions, Daniell Kernel, $\omega=0, \quad p=0, b=0.04$

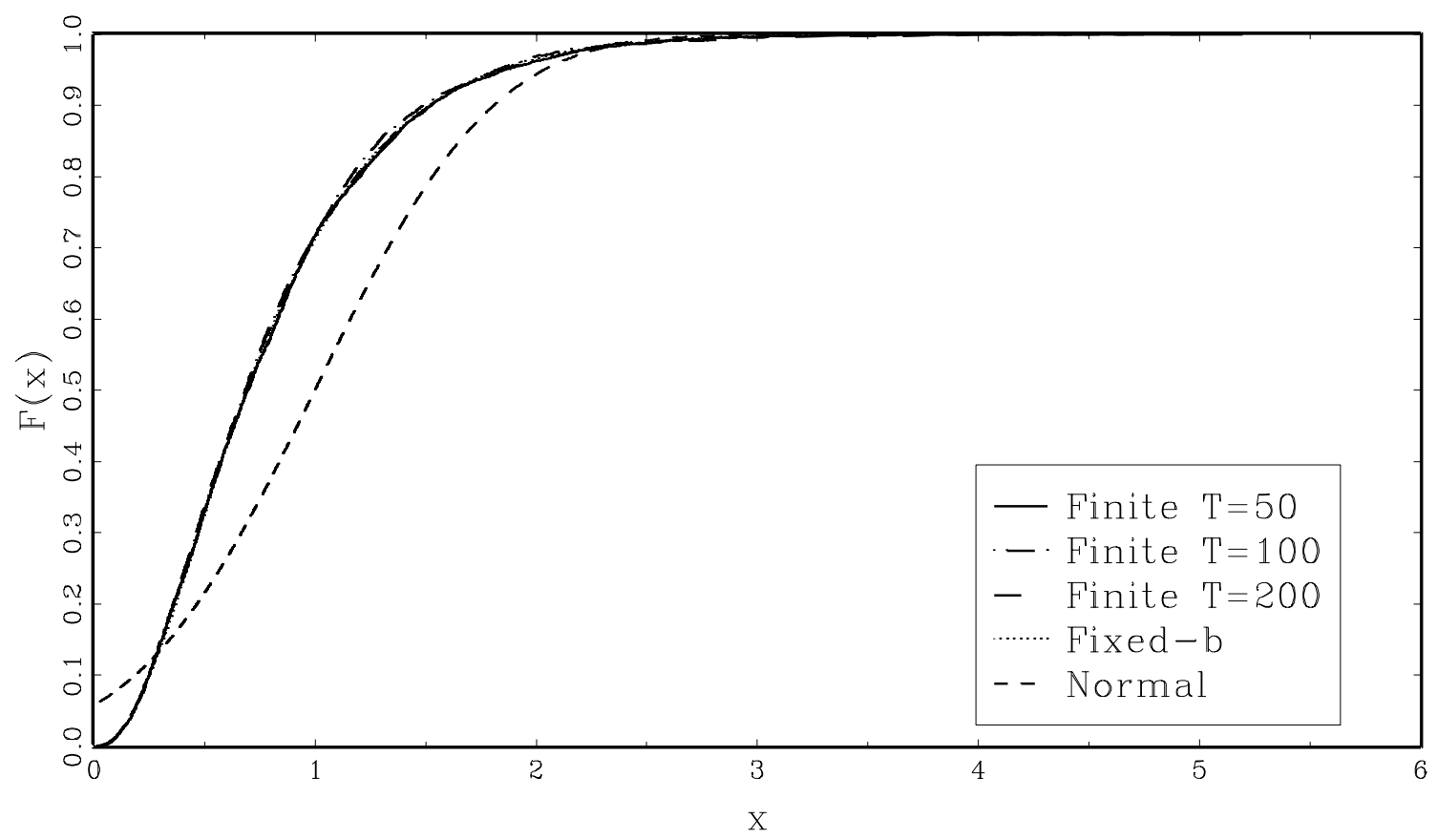

Figure 3.2: CDF Functions, Daniell Kernel, $\omega=0, p=0, \quad b=0.2$ 


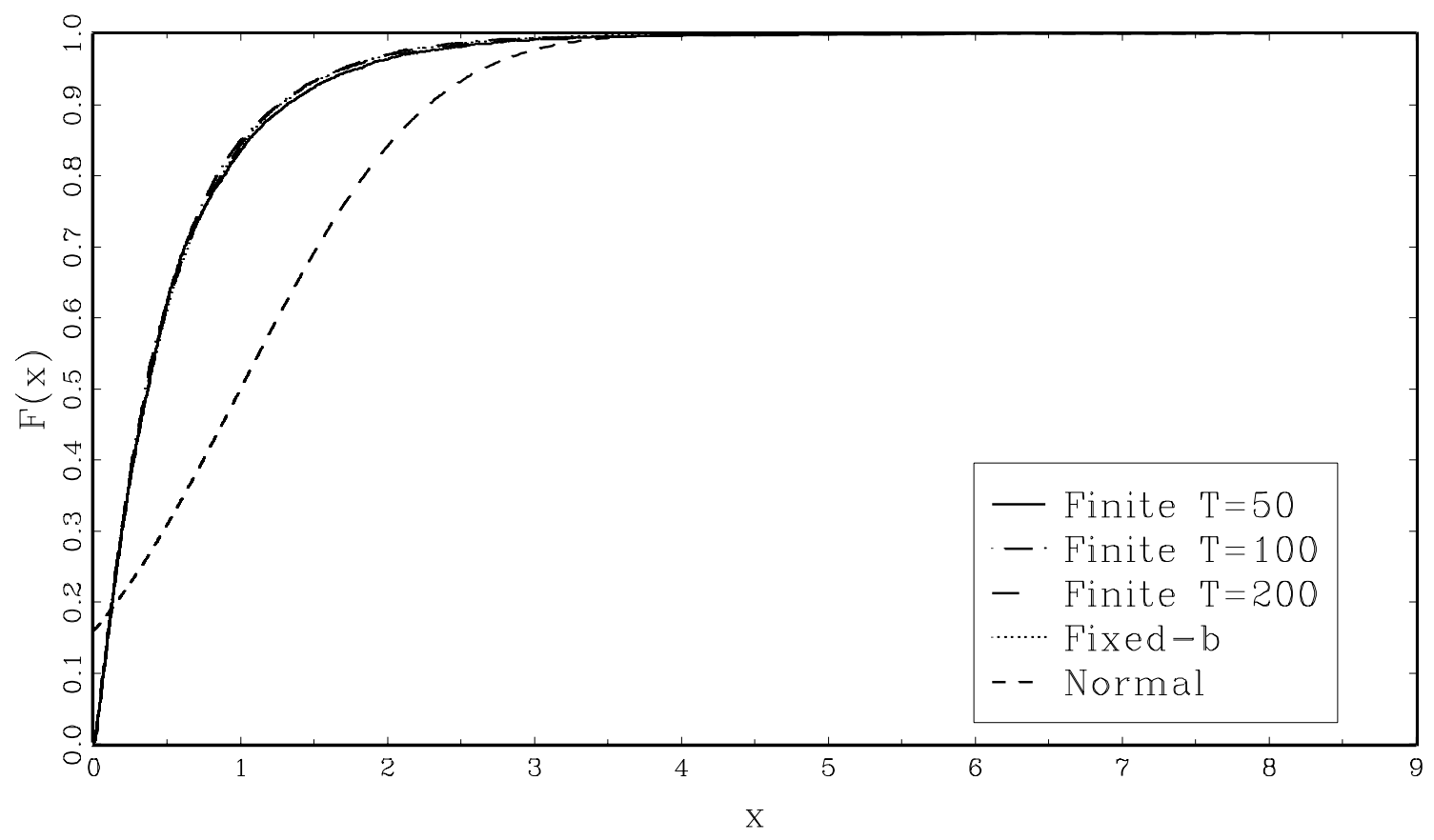

Figure 3.3: CDF Functions, Daniell Kernel, $\omega=0, p=0, b=0.5$

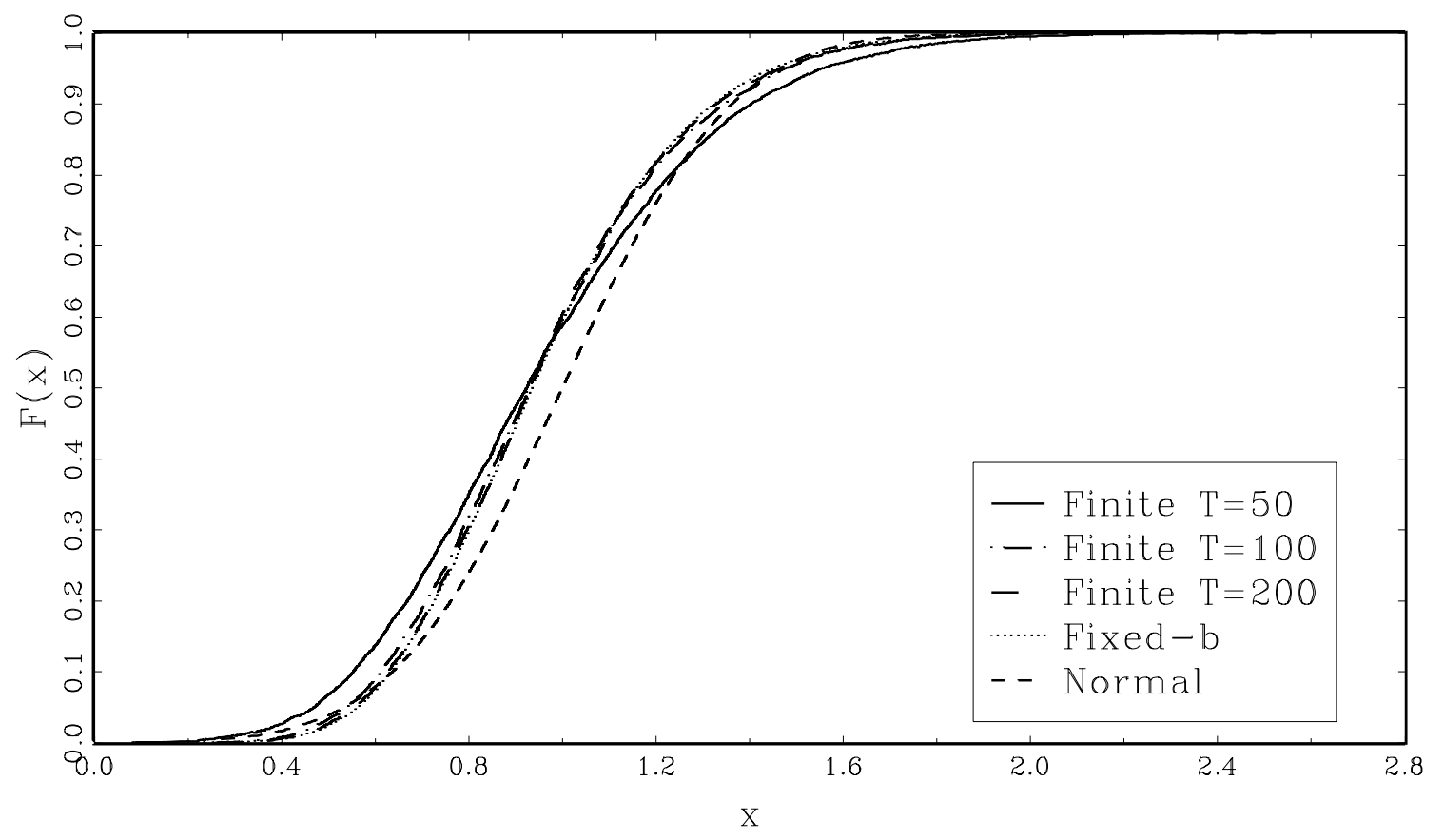

Figure 3.4: CDF Functions, Daniell Kernel, $\omega=0, p=-0.4, b=0.04$ 


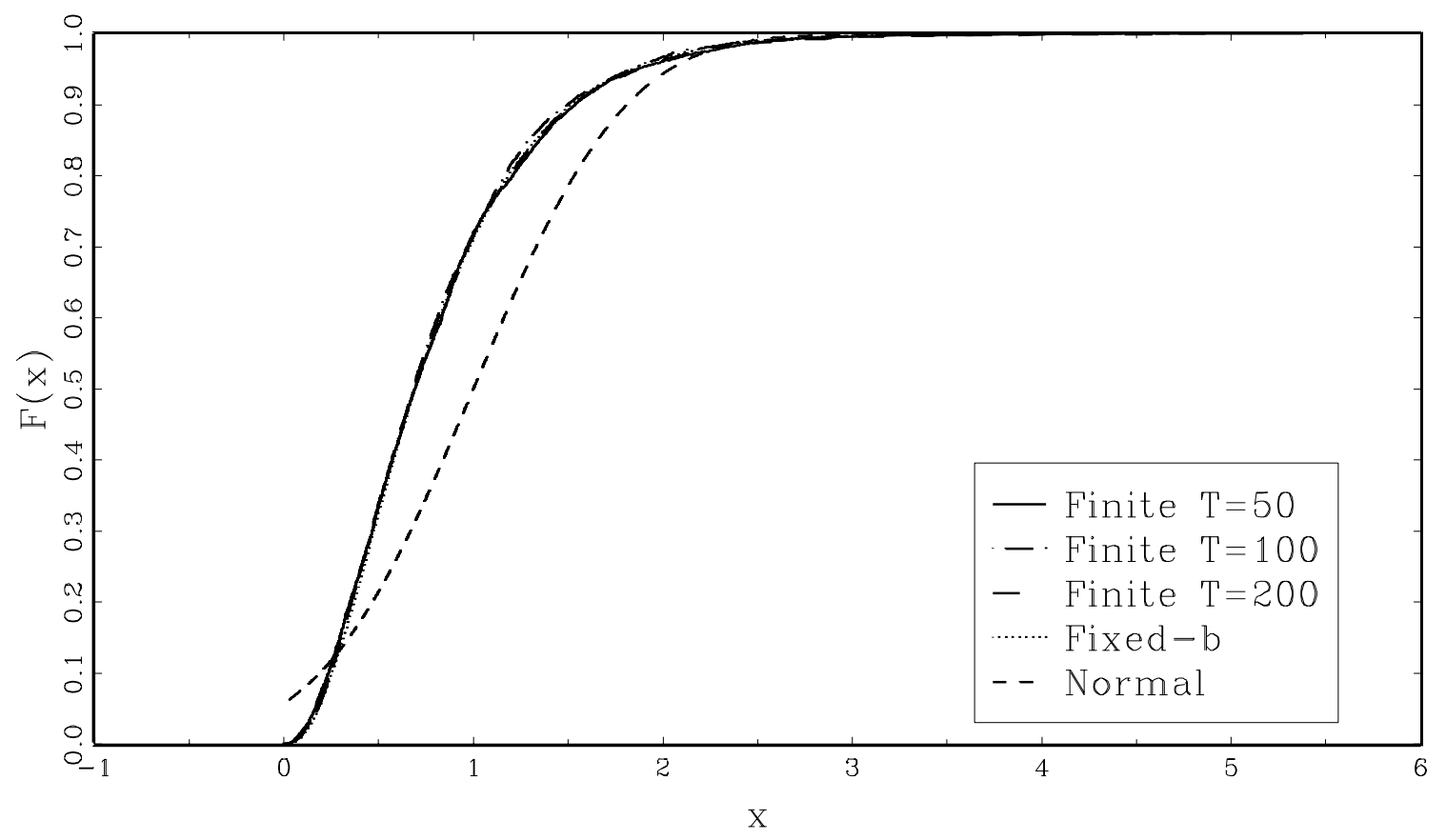

Figure 3.5: CDF Functions, Daniell Kernel, $\omega=0, \rho=-0.4, b=0.2$

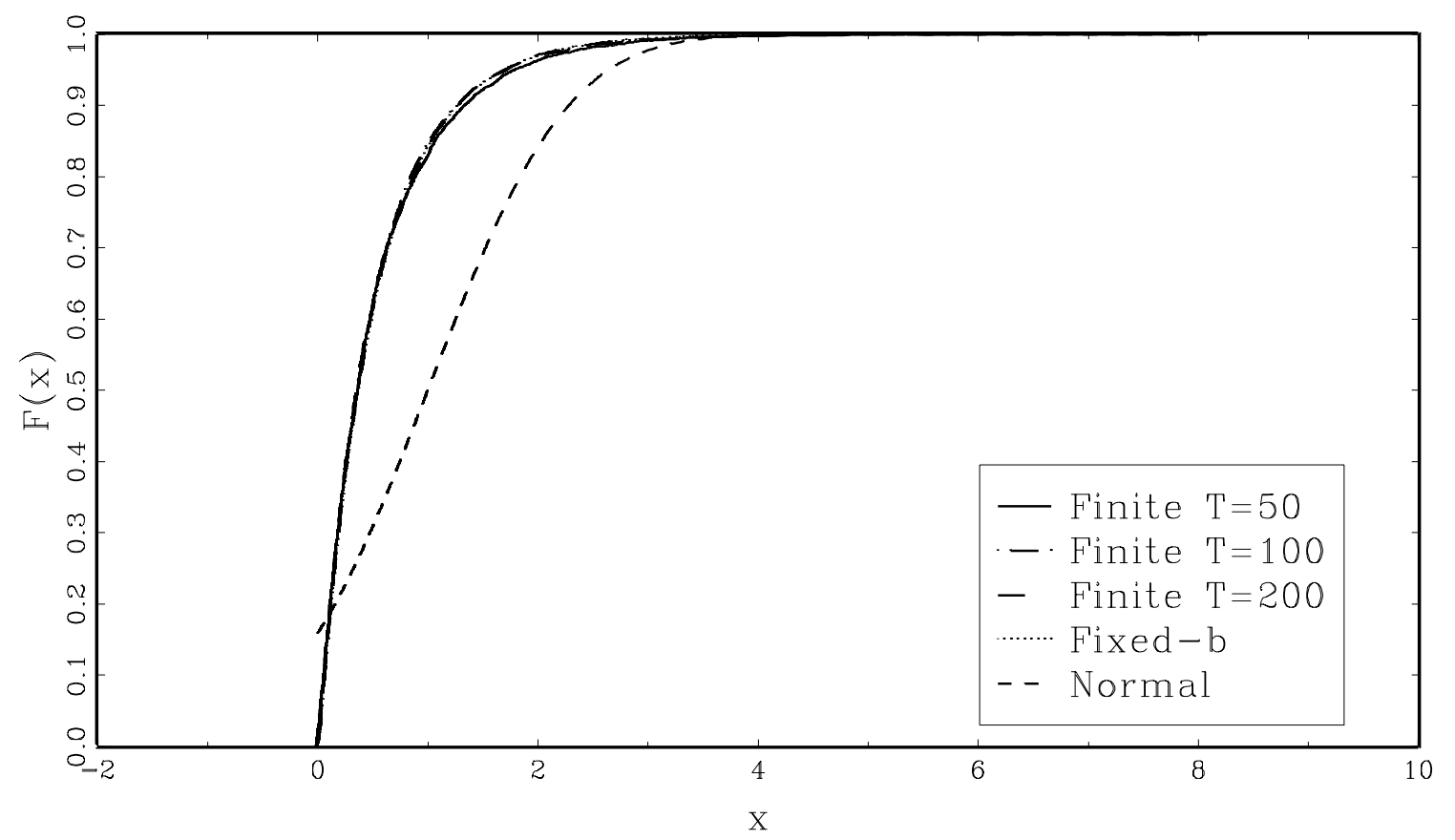

Figure 3.6: CDF Functions, Daniell Kernel, $\omega=0, \rho=-0.4, b=0.5$ 


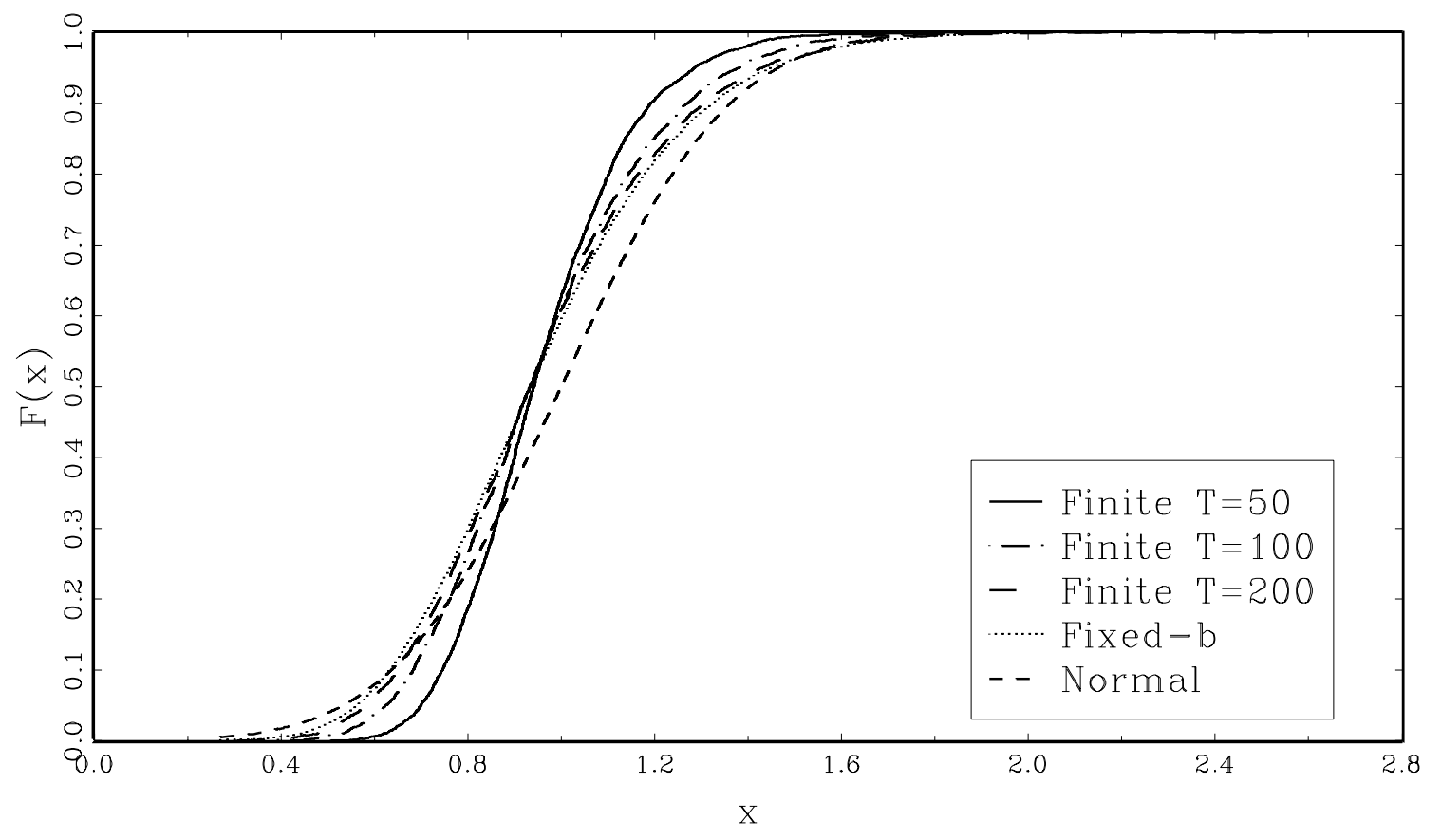

Figure 3.7: CDF Functions, Daniell Kernel, $\omega=0, p=0.4, \quad b=0.04$

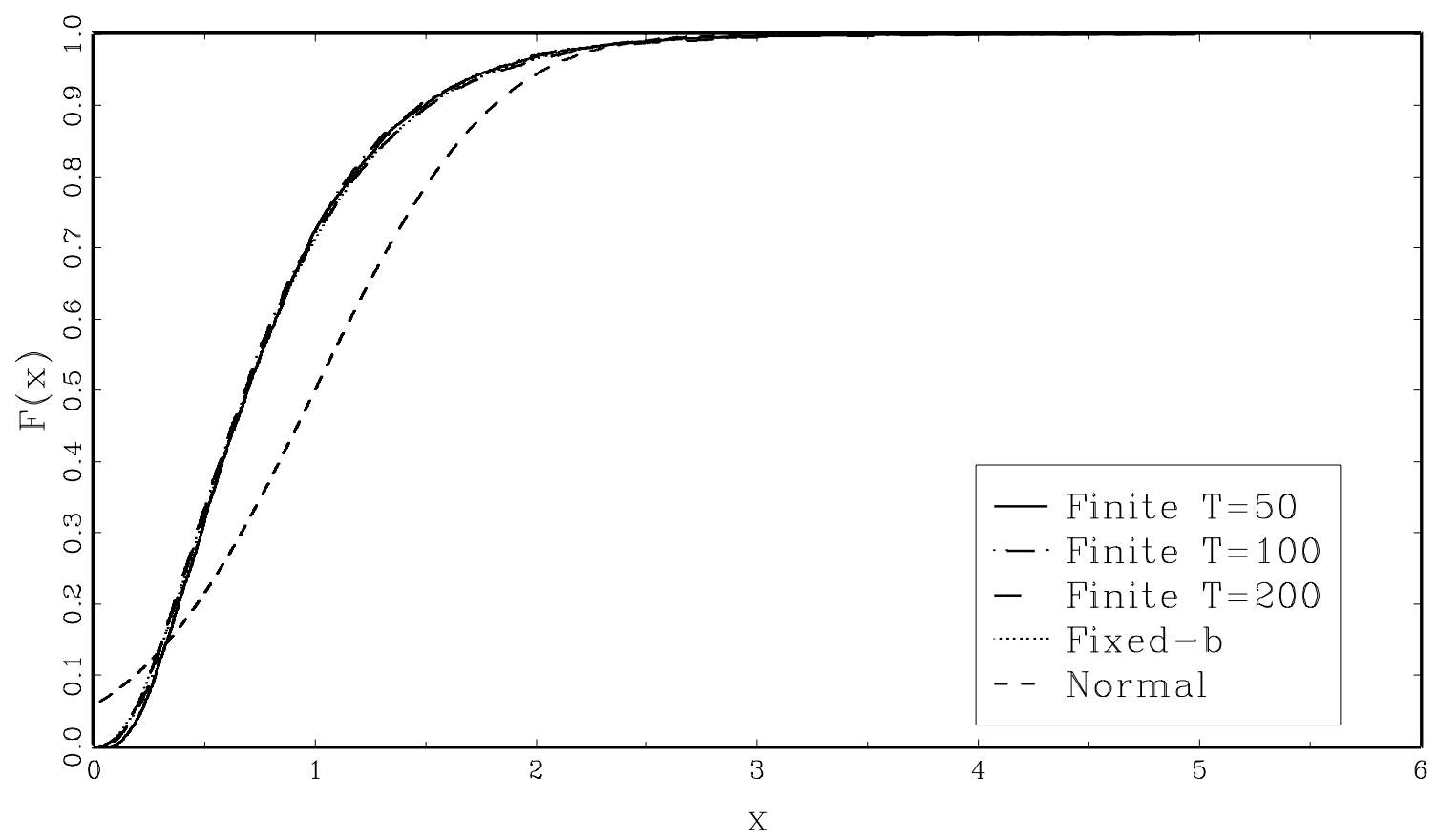

Figure 3.8: CDF Functions, Daniell Kernel, $\omega=0, \rho=0.4, \quad b=0.2$ 


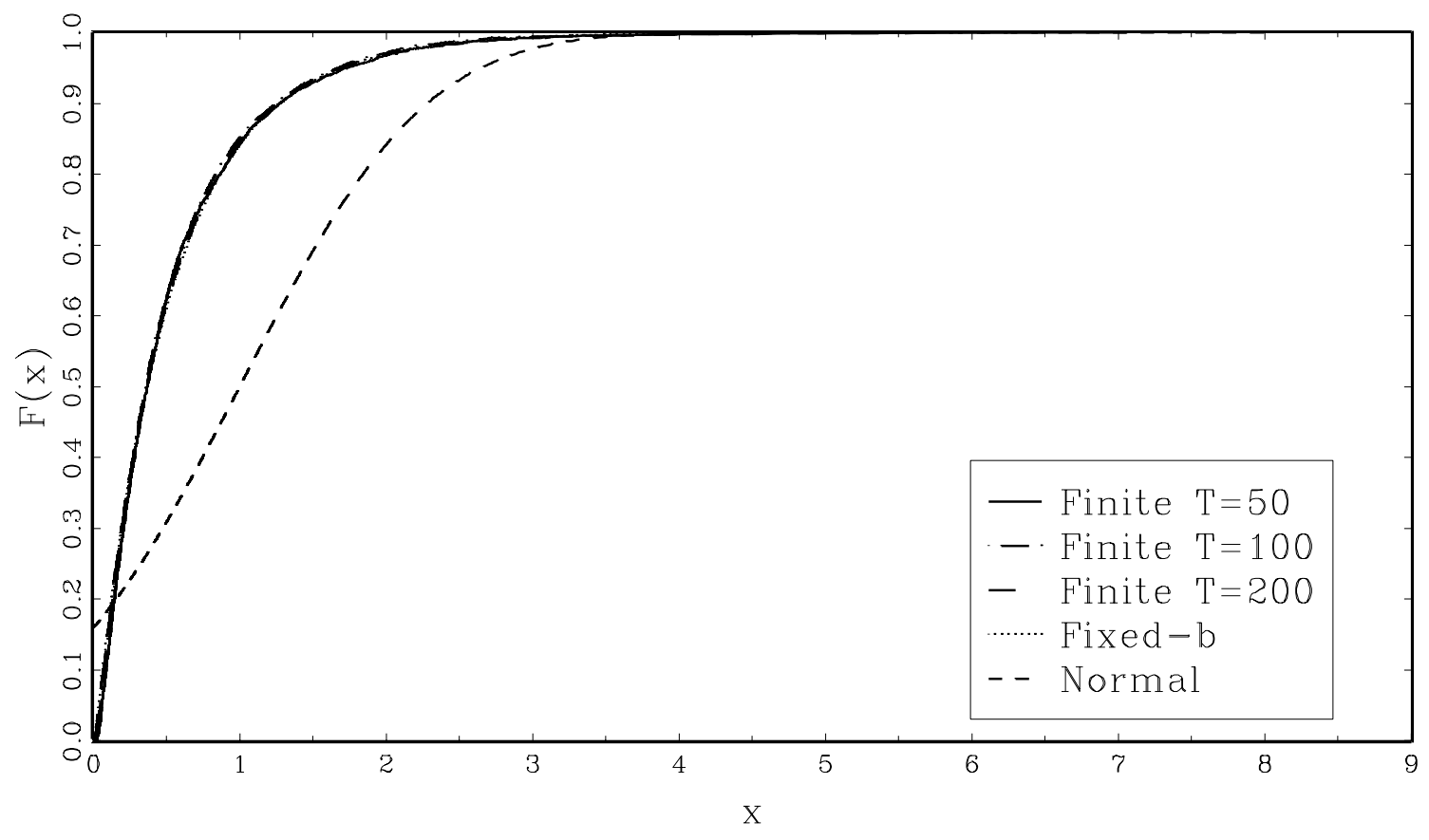

Figure 3.9: CDF Functions, Daniell Kernel, $\omega=0, \rho=0.4, \quad b=0.5$

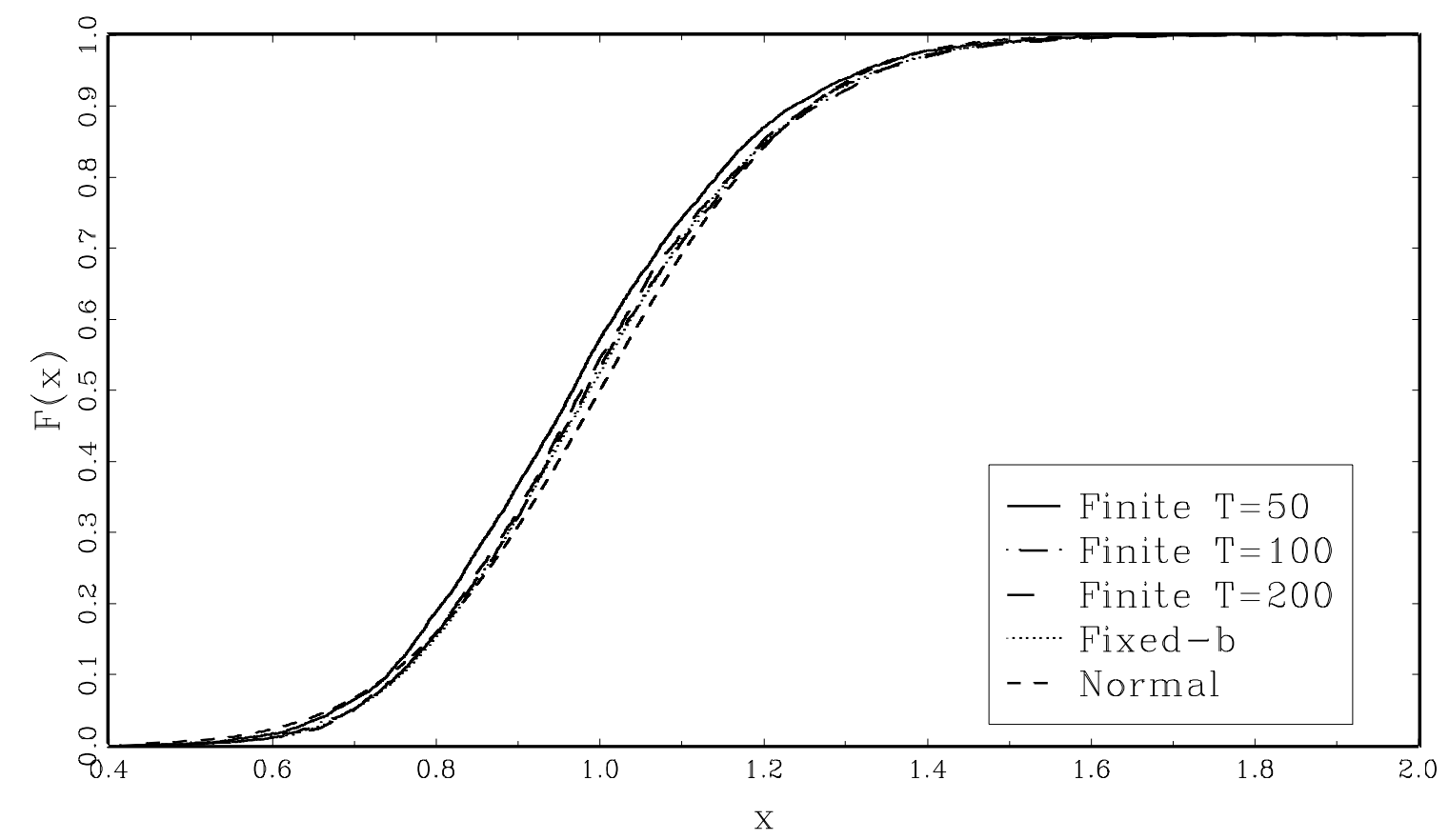

Figure 3.10: CDF Functions, Daniell Kernel, $\omega=0.5 \pi, \rho=0.0, b=0.04$ 


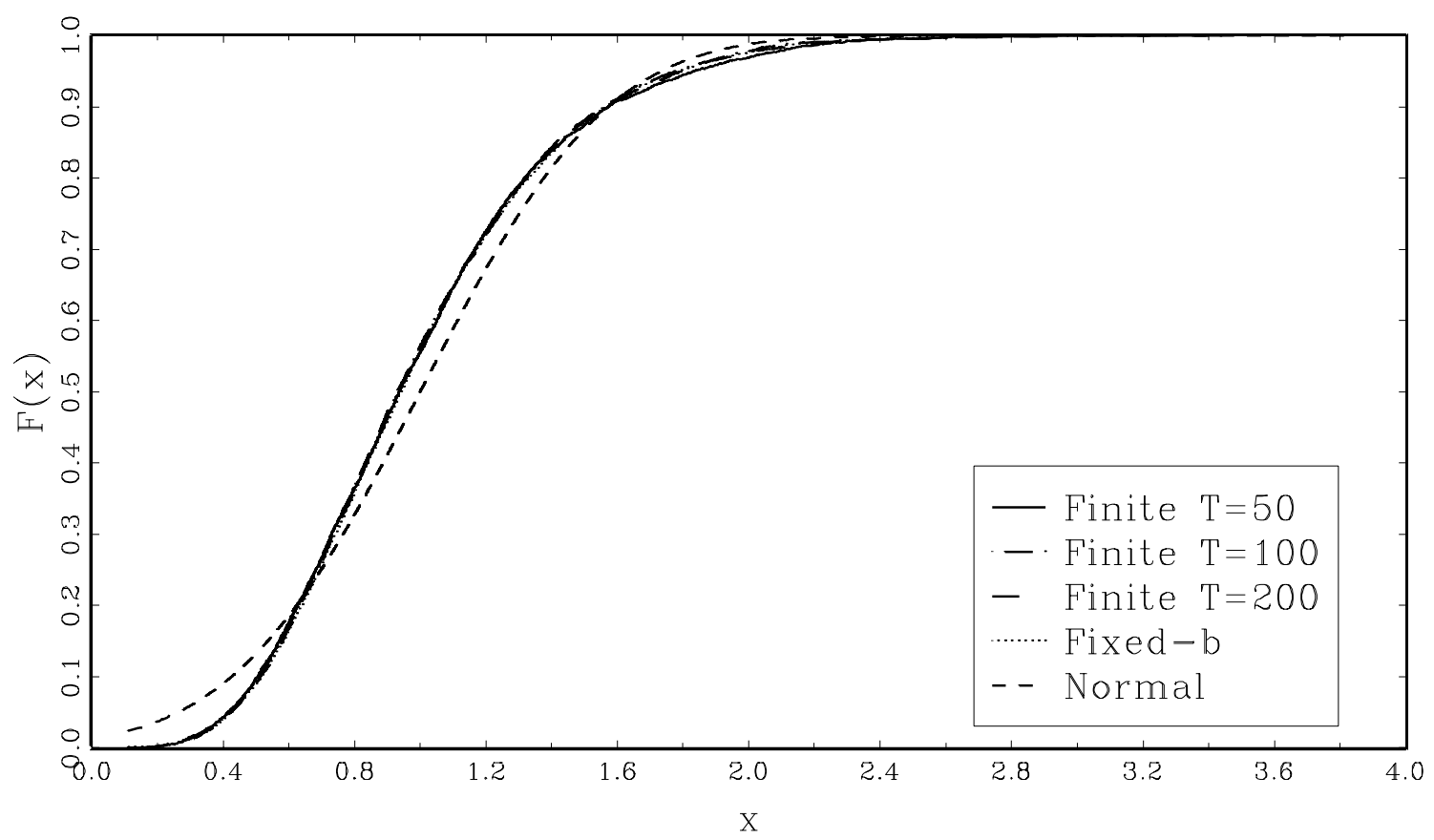

Figure 3.11: CDF Functions, Daniell Kernel, $\omega=0.5 \pi, \quad \rho=0.0, \quad b=0.2$

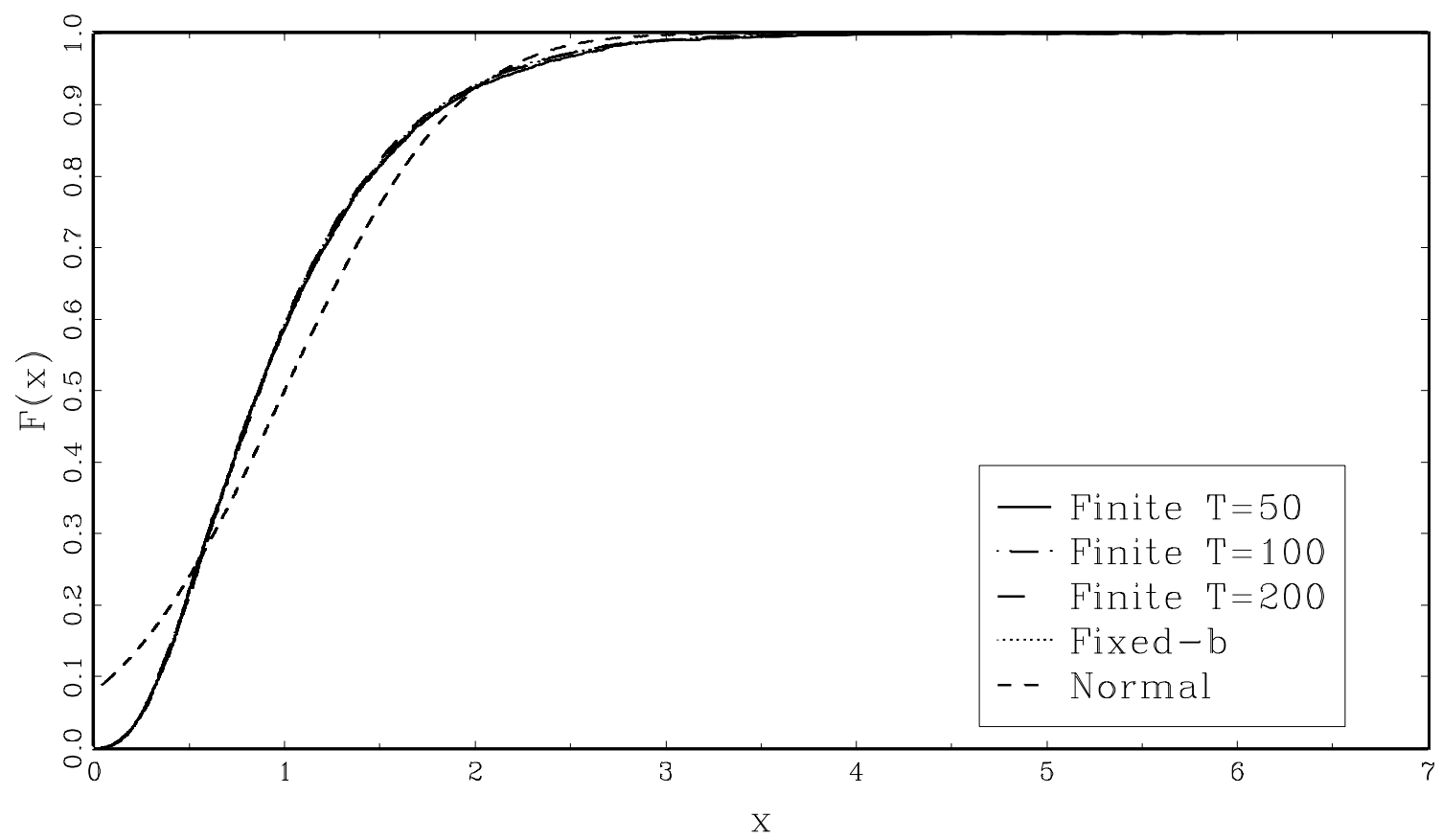

Figure 3.12: CDF Functions, Daniell Kernel, $\omega=0.5 \pi, \rho=0.0, \quad b=0.5$ 


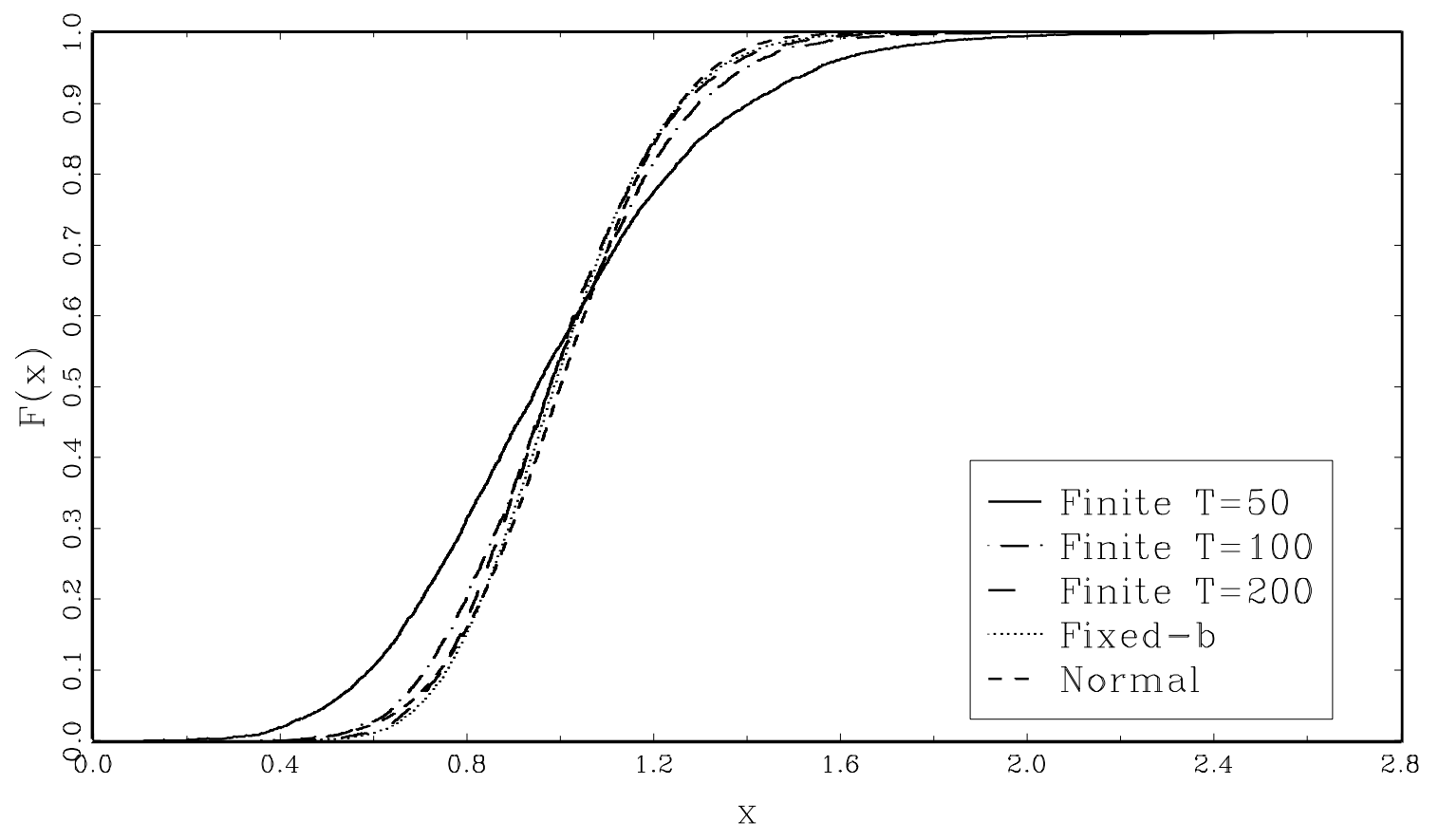

Figure 3.13: CDF Functions, Daniell Kernel, $\omega=0.5 \pi, \rho=-0.4, b=0.04$

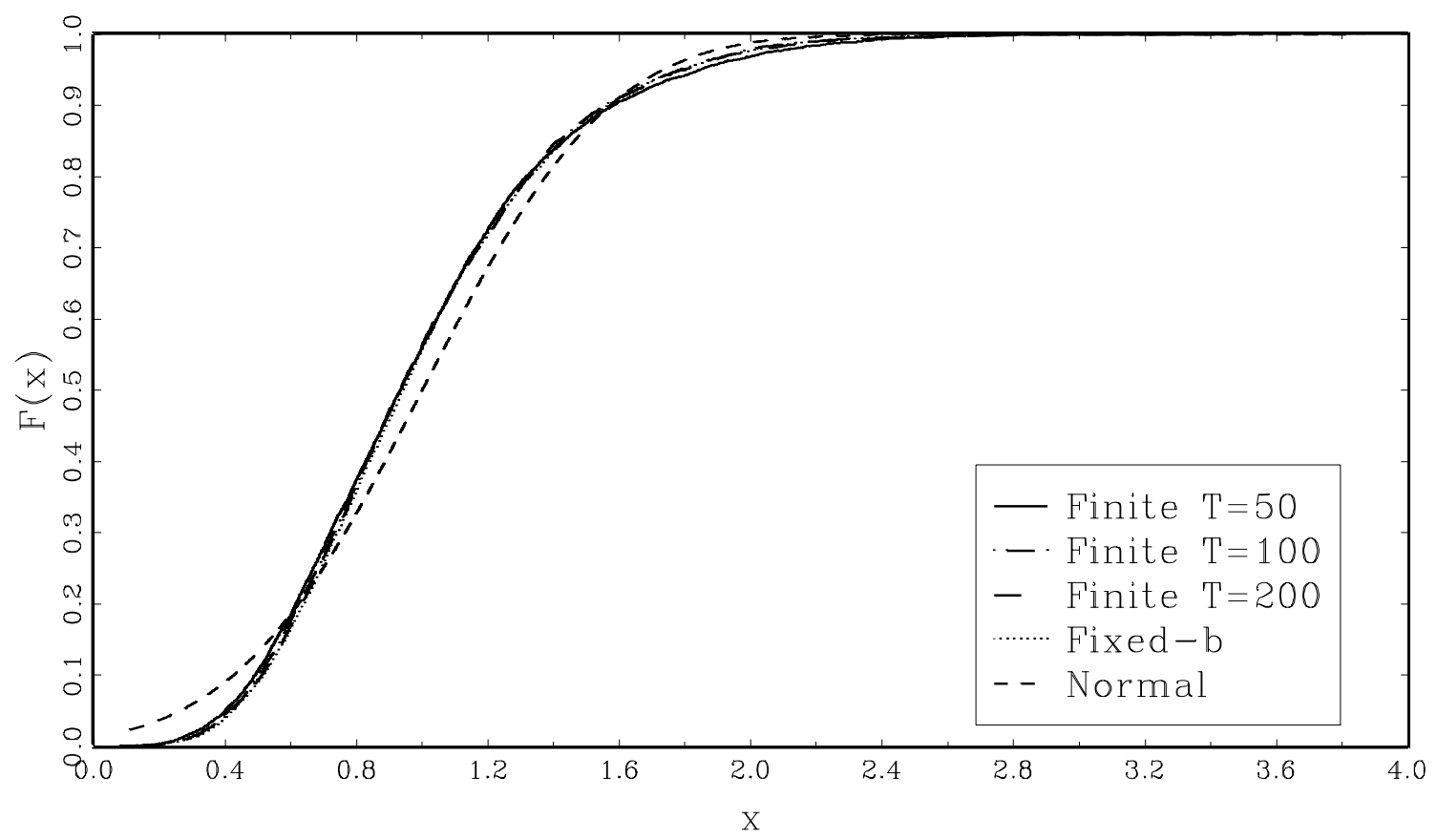

Figure 3.14: CDF Functions, Daniell Kernel, $\omega=0.5 \pi, \rho=-0.4, \quad b=0.2$ 


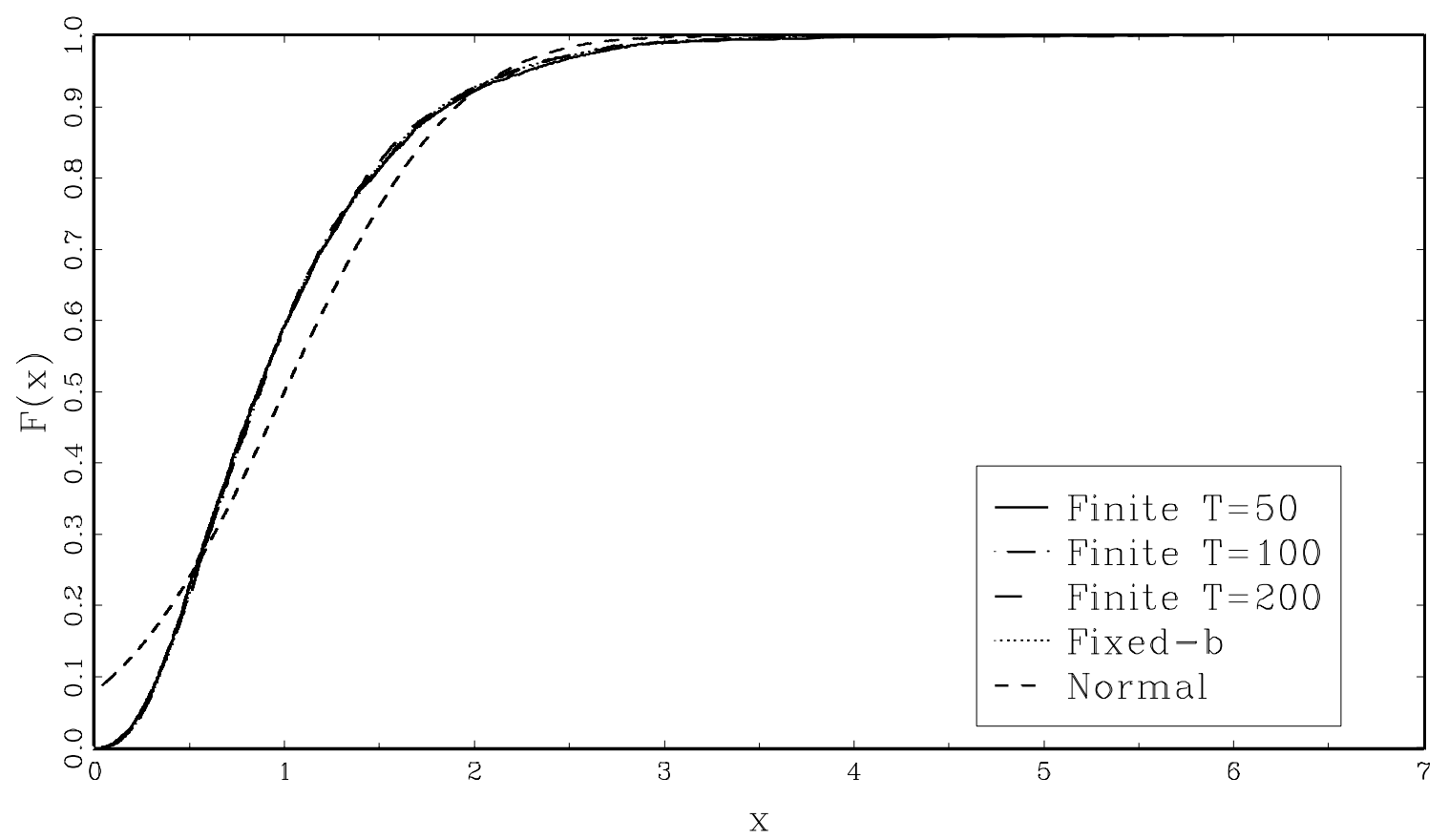

Figure 3.15: CDF Functions, Daniell Kernel, $\omega=0.5 \pi, p=-0.4, b=0.5$

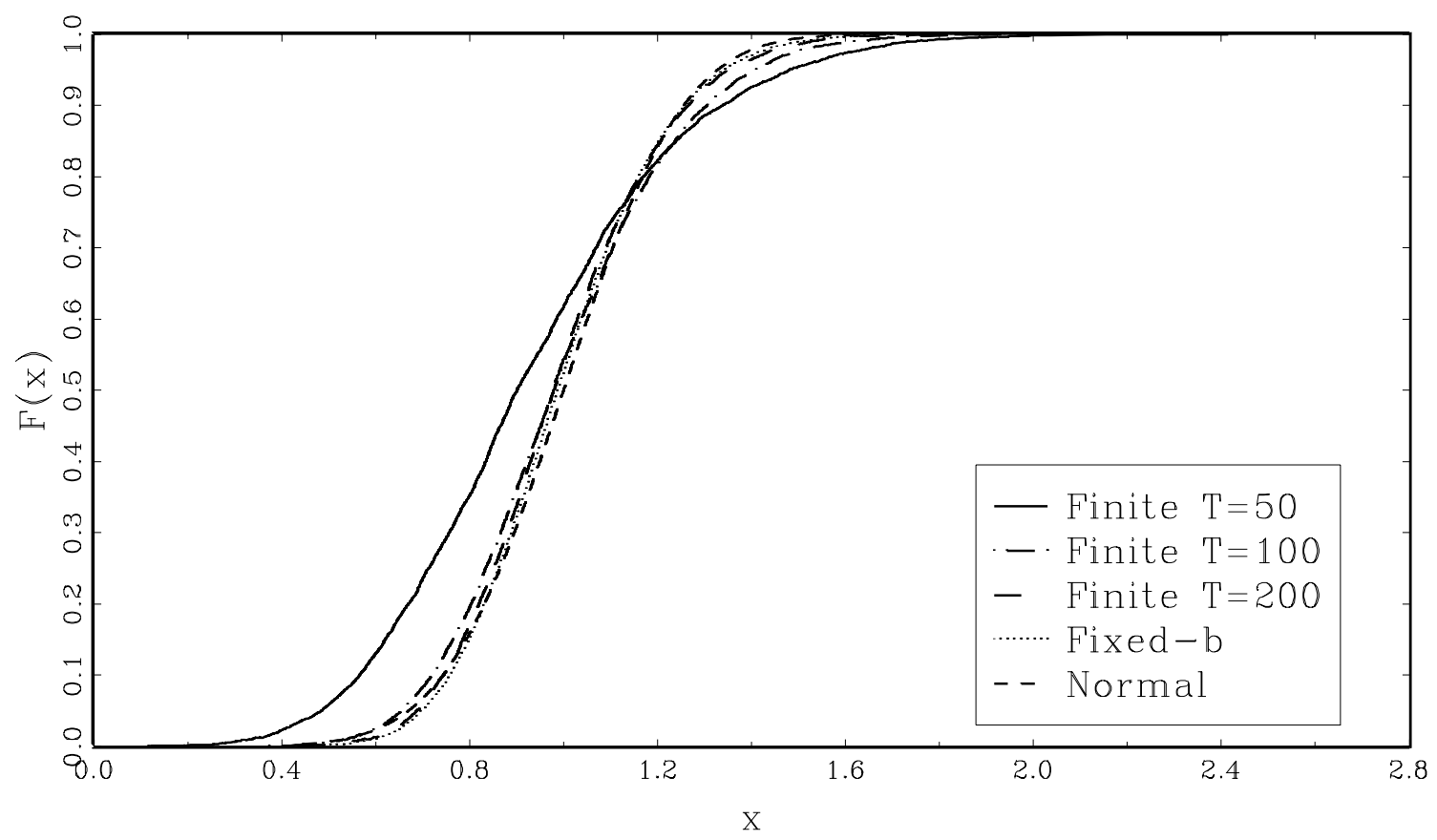

Figure 3.16: CDF Functions, Daniell Kernel, $\omega=0.5 \pi, \rho=0.4, b=0.04$ 


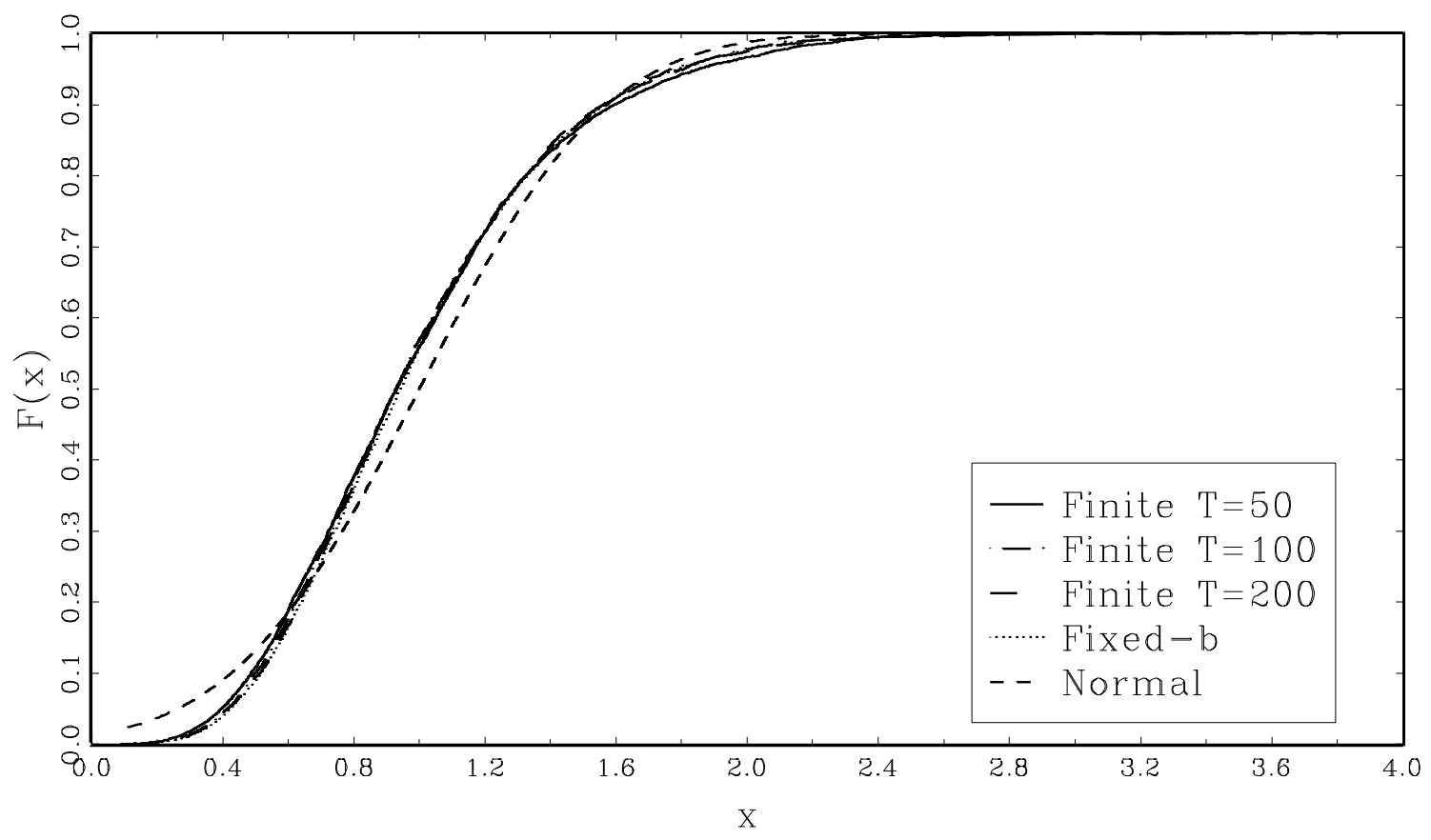

Figure 3.17: CDF Functions, Daniell Kernel, $\omega=0.5 \pi, p=-0.4, b=0.2$

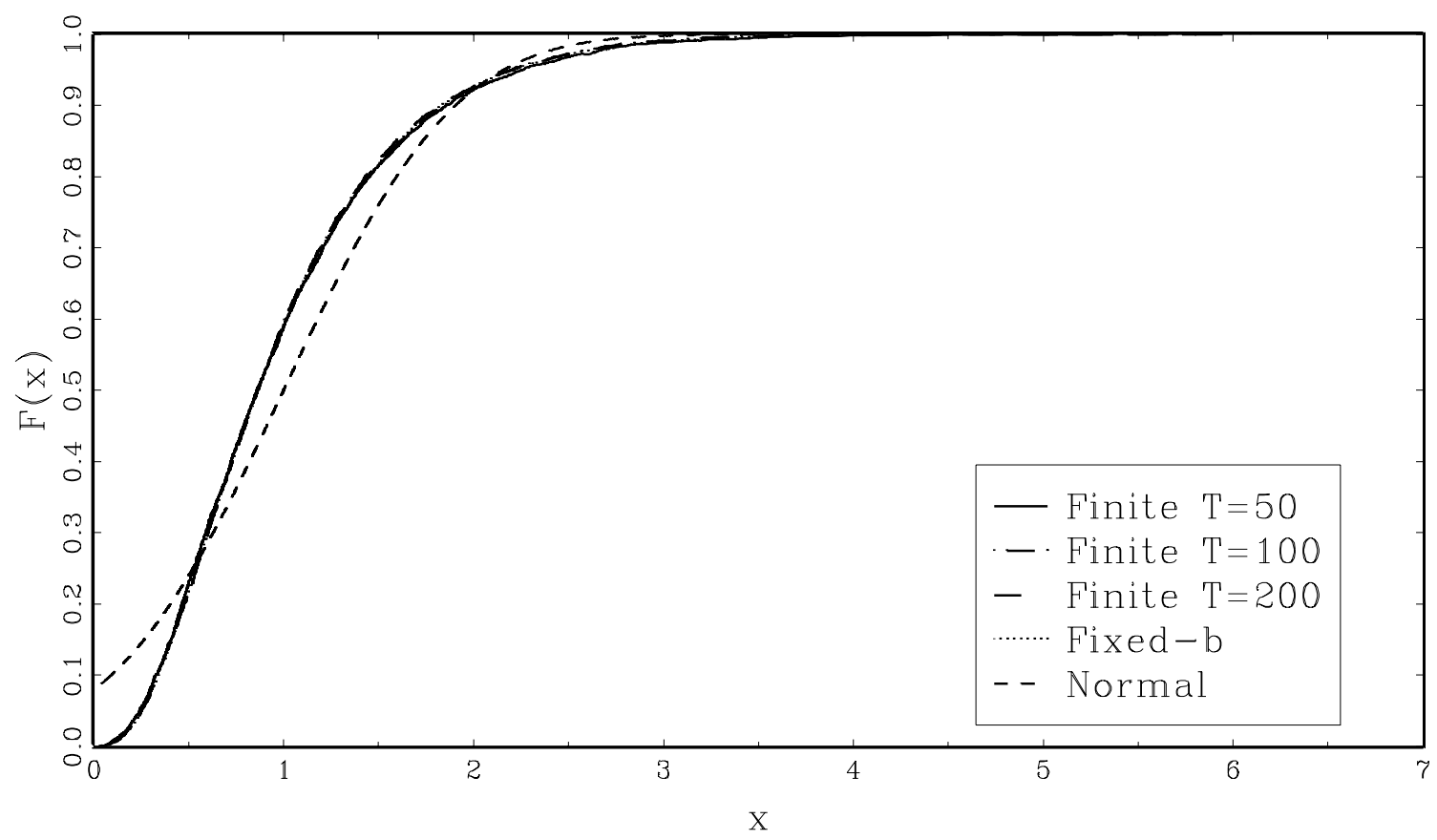

Figure 3.18: CDF Functions, Daniell Kernel, $\omega=0.5 \pi, \rho=0.4, \quad b=0.5$ 


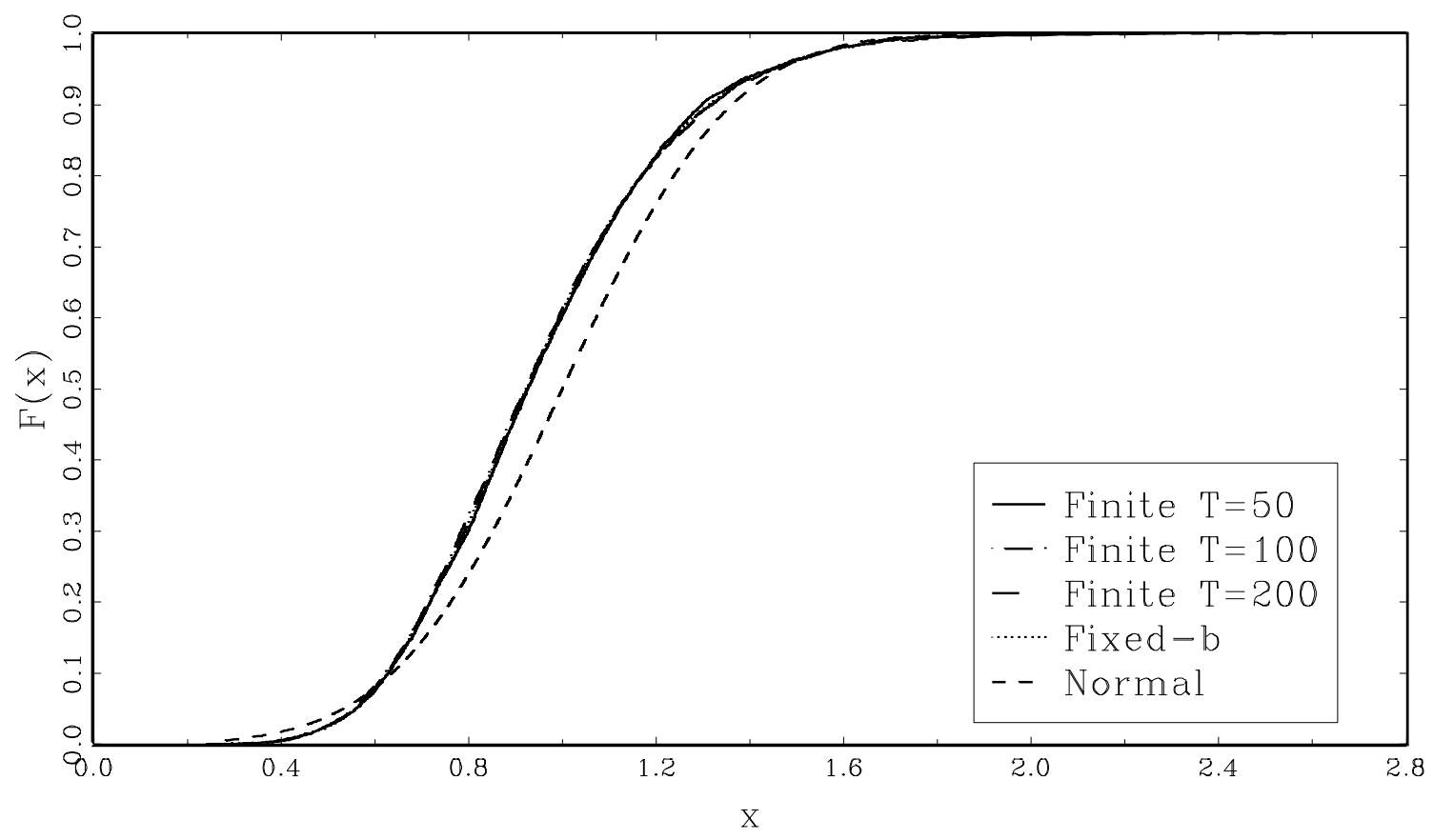

Figure 4.1: CDF Functions, QS Kernel, $\omega=0, \rho=0, \quad b=0.04$

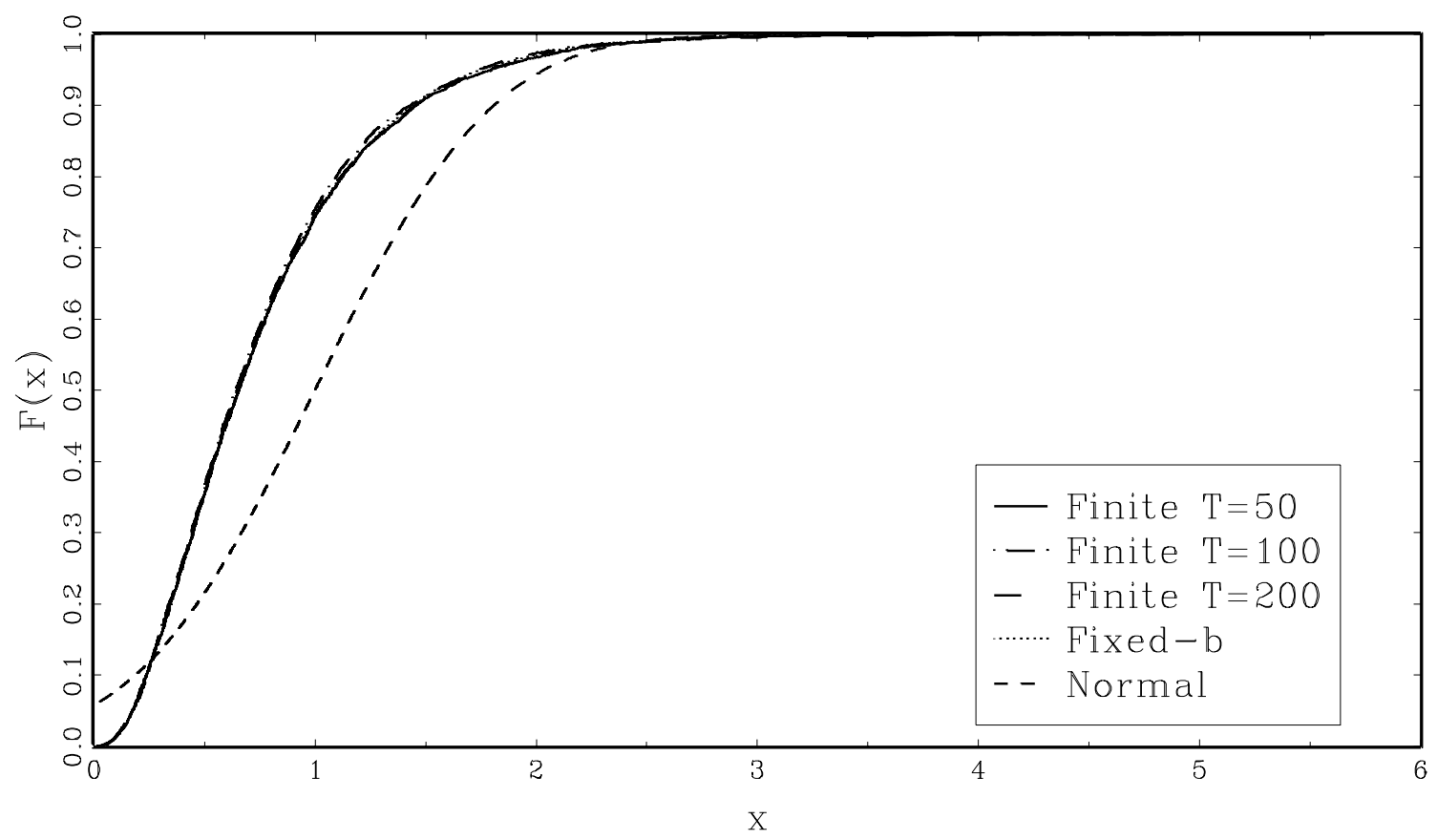

Figure 4.2: CDF Functions, QS Kernel, $\omega=0, \quad \rho=0, \quad b=0.2$ 


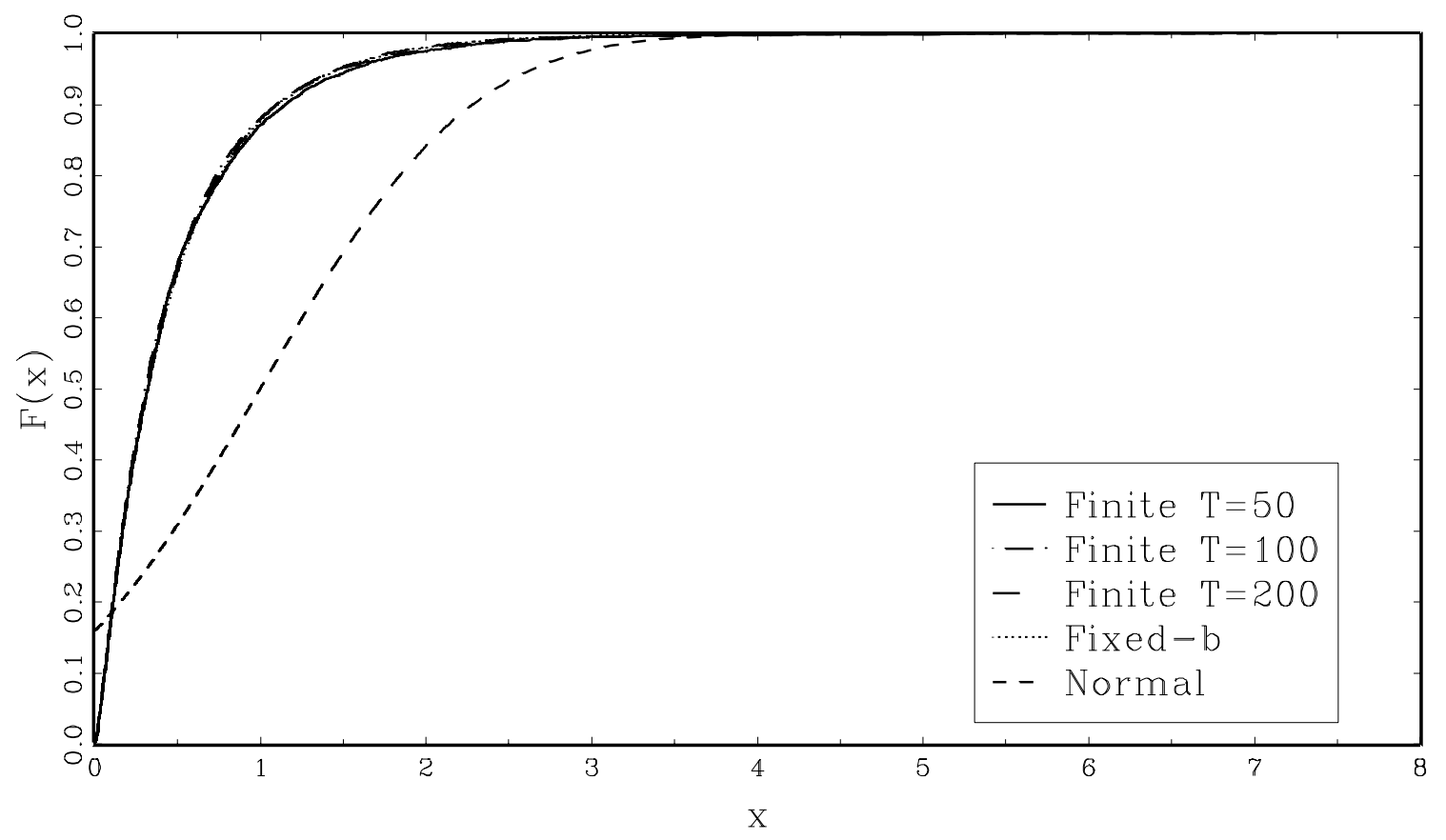

Figure 4.3: CDF Functions, QS Kernel, $\omega=0, \rho=0, \quad b=0.5$

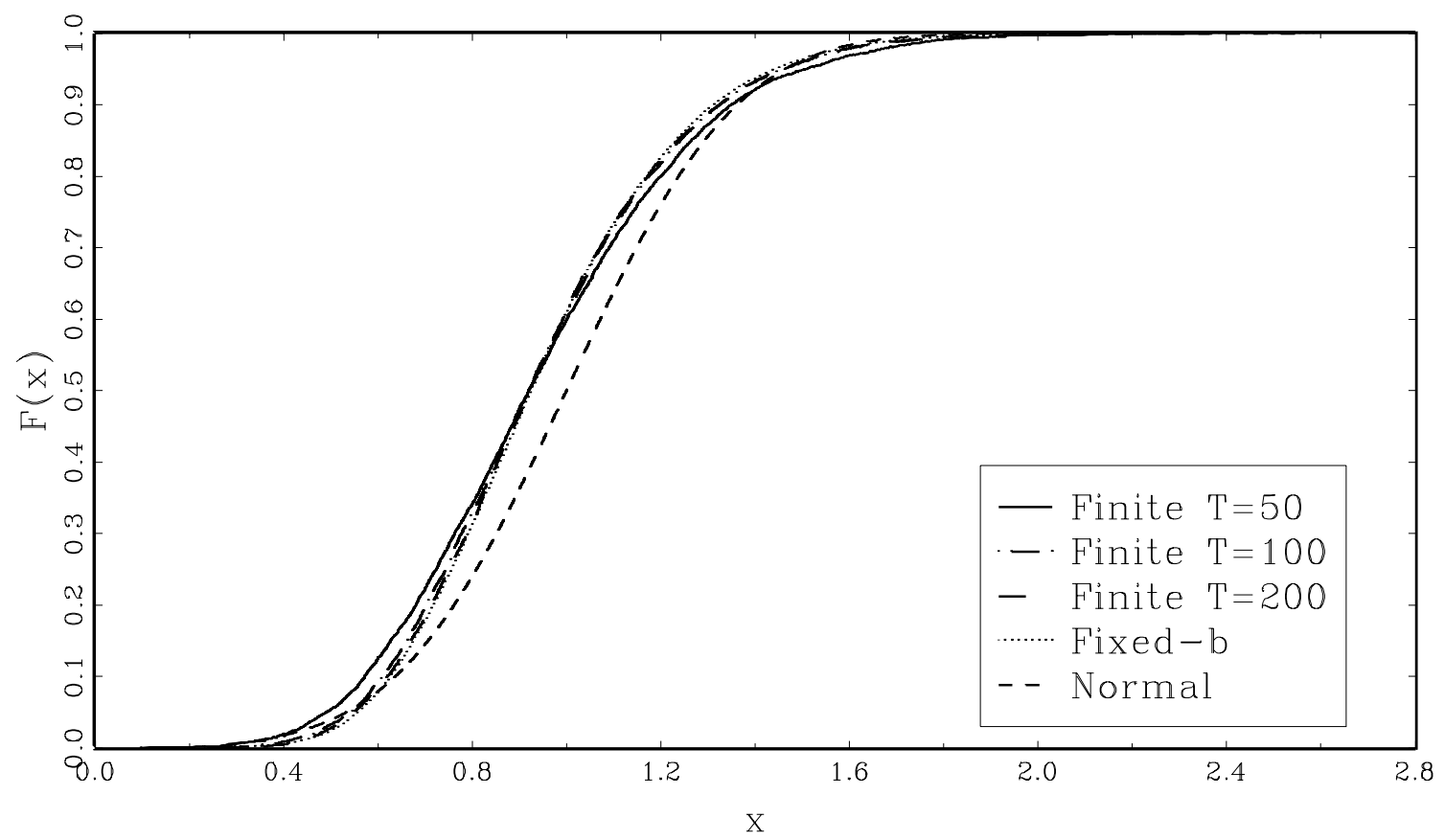

Figure 4.4: CDF Functions, QS Kernel, $\omega=0, \rho=-0.4, b=0.04$ 


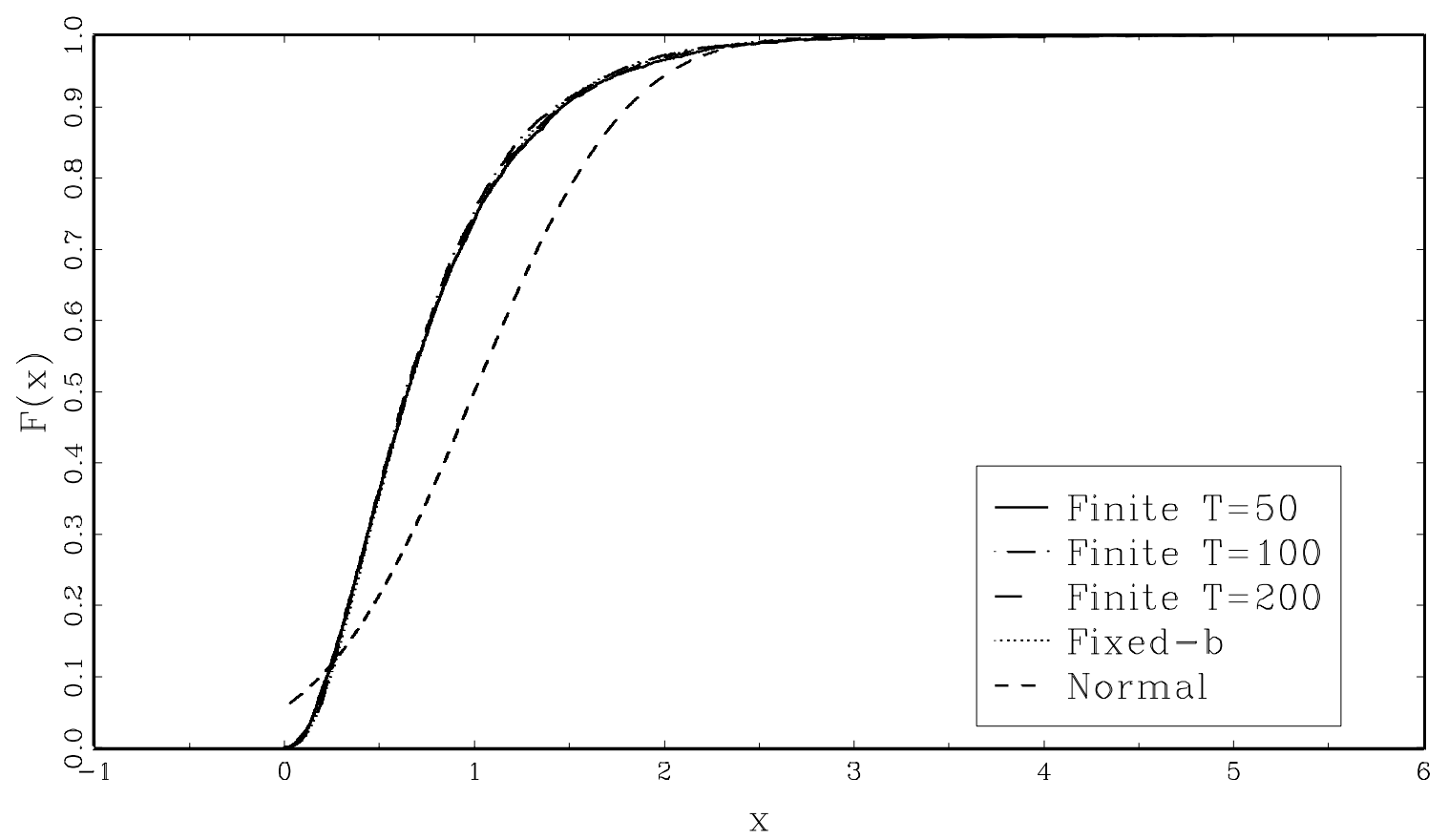

Figure 4.5: CDF Functions, QS Kernel, $\omega=0, p=-0.4, b=0.2$

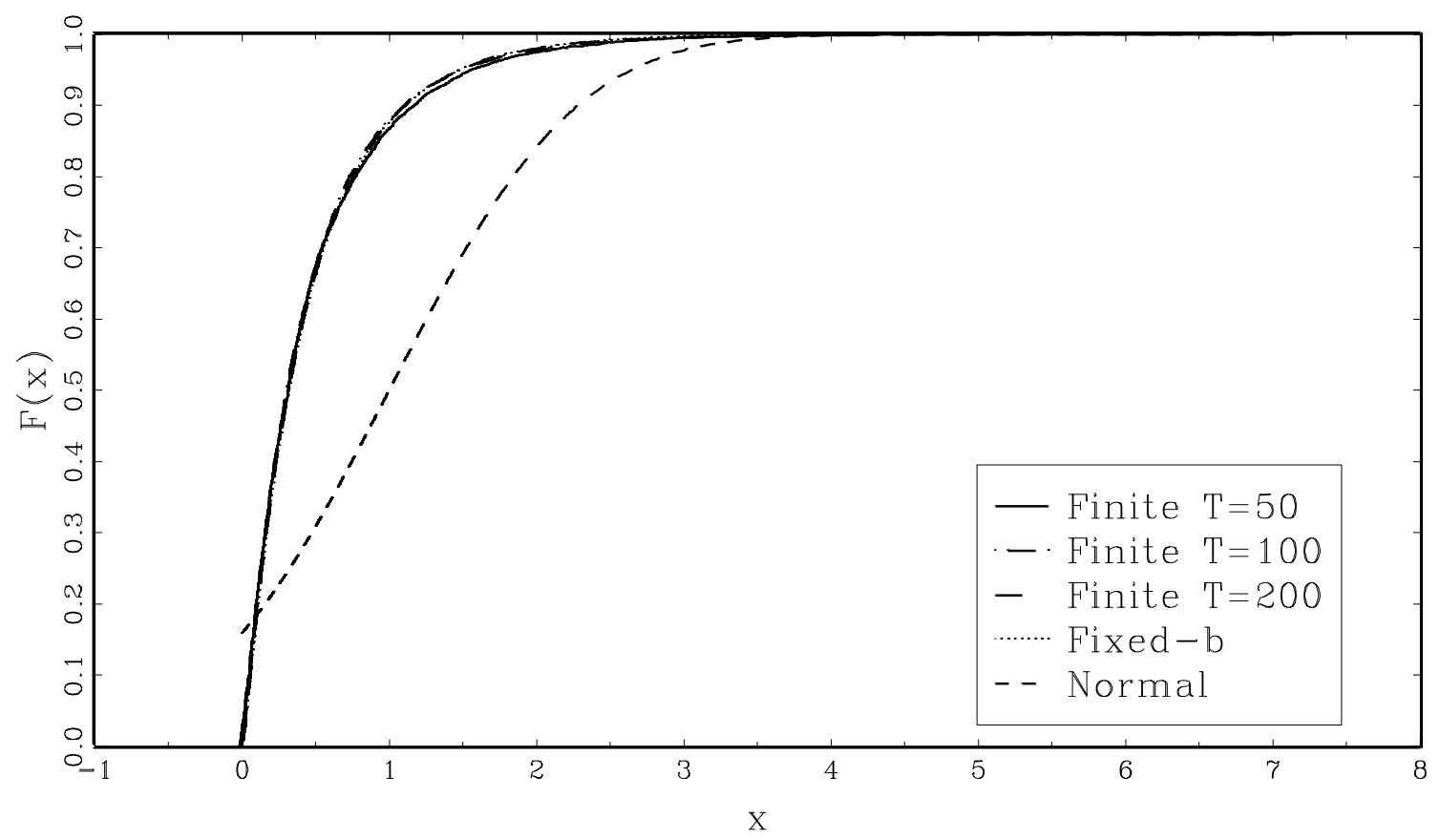

Figure 4.6: CDF Functions, QS Kernel, $\omega=0, \quad \rho=-0.4, \quad b=0.5$ 


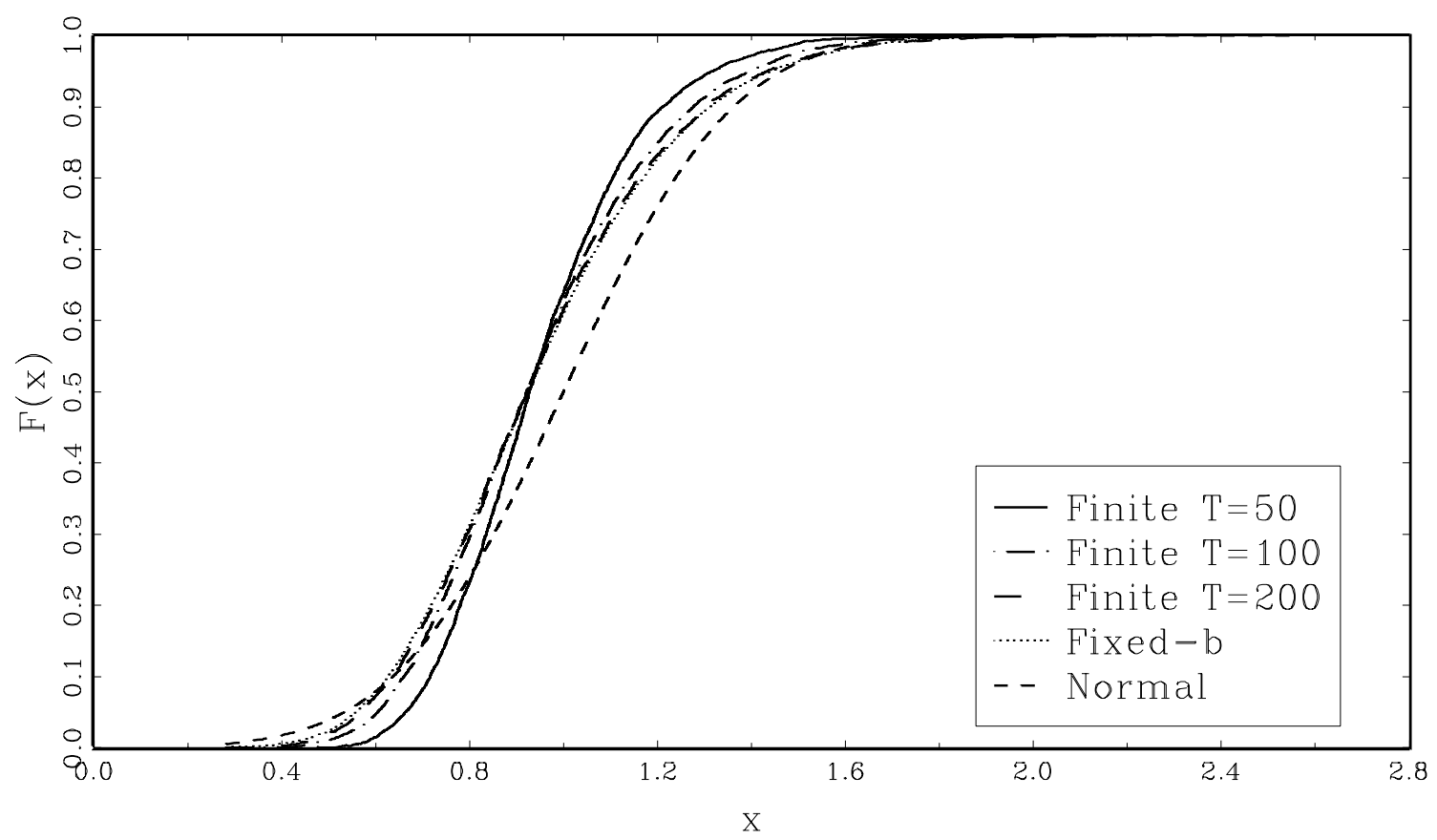

Figure 4.7: CDF Functions, QS Kernel, $\omega=0, \rho=0.4, \quad b=0.04$

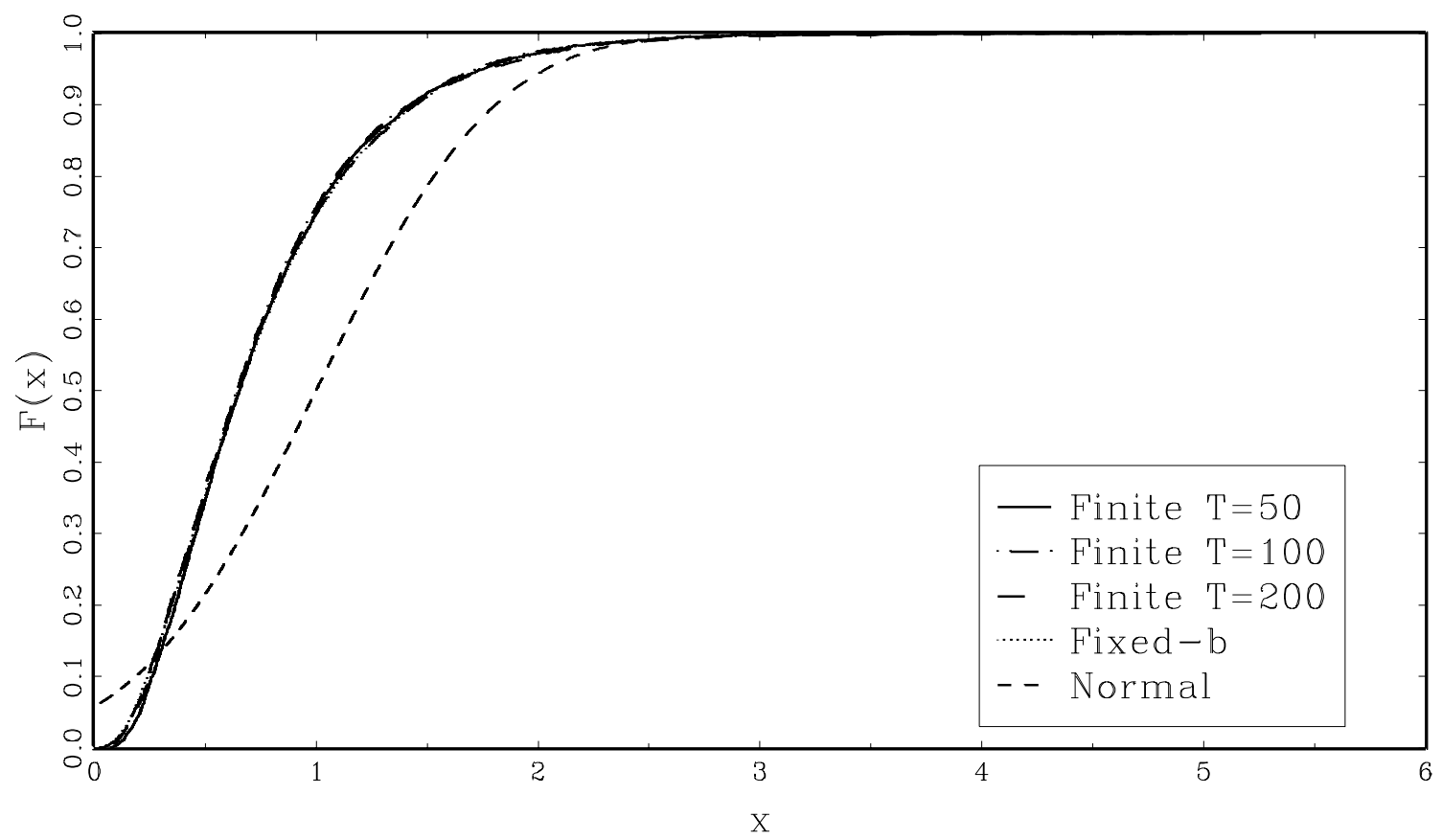

Figure 4.8: CDF Functions, QS Kernel, $\omega=0, \rho=0.4, b=0.2$ 


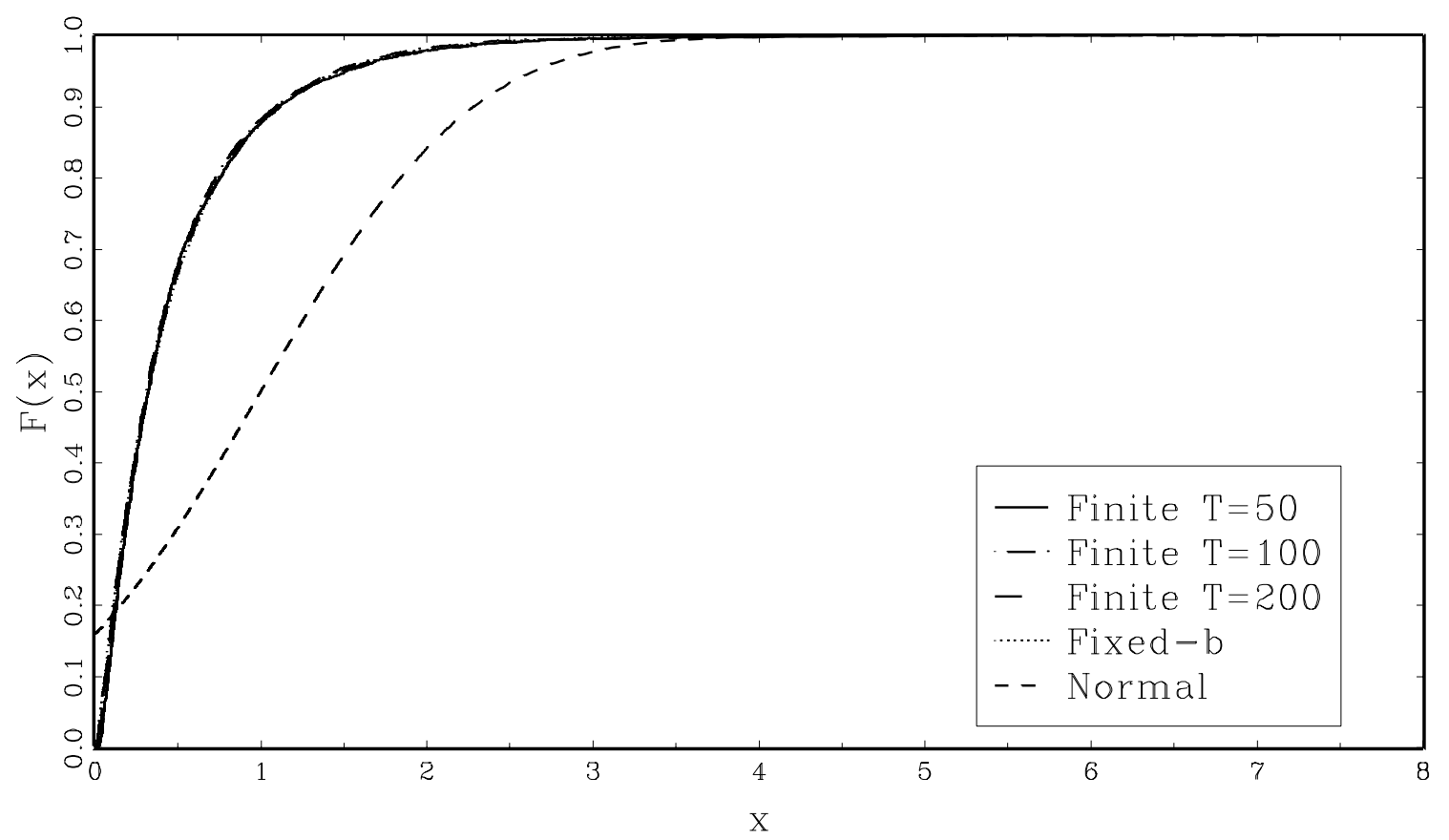

Figure 4.9: CDF Functions, QS Kernel, $\omega=0, \rho=0.4, b=0.5$

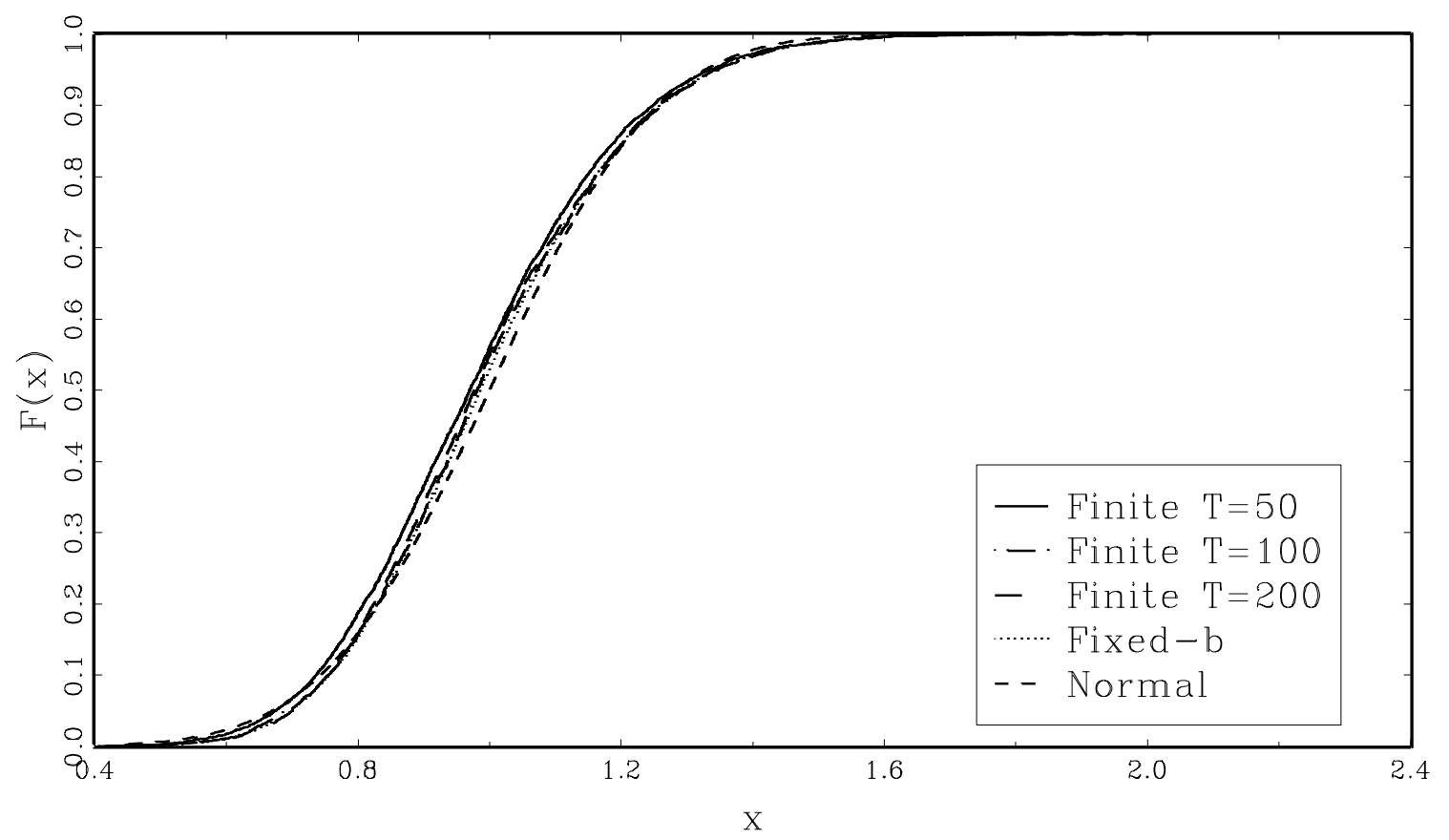

Figure 4.10: CDF Functions, QS Kernel, $\omega=0.5 \pi, \rho=0.0, b=0.04$ 


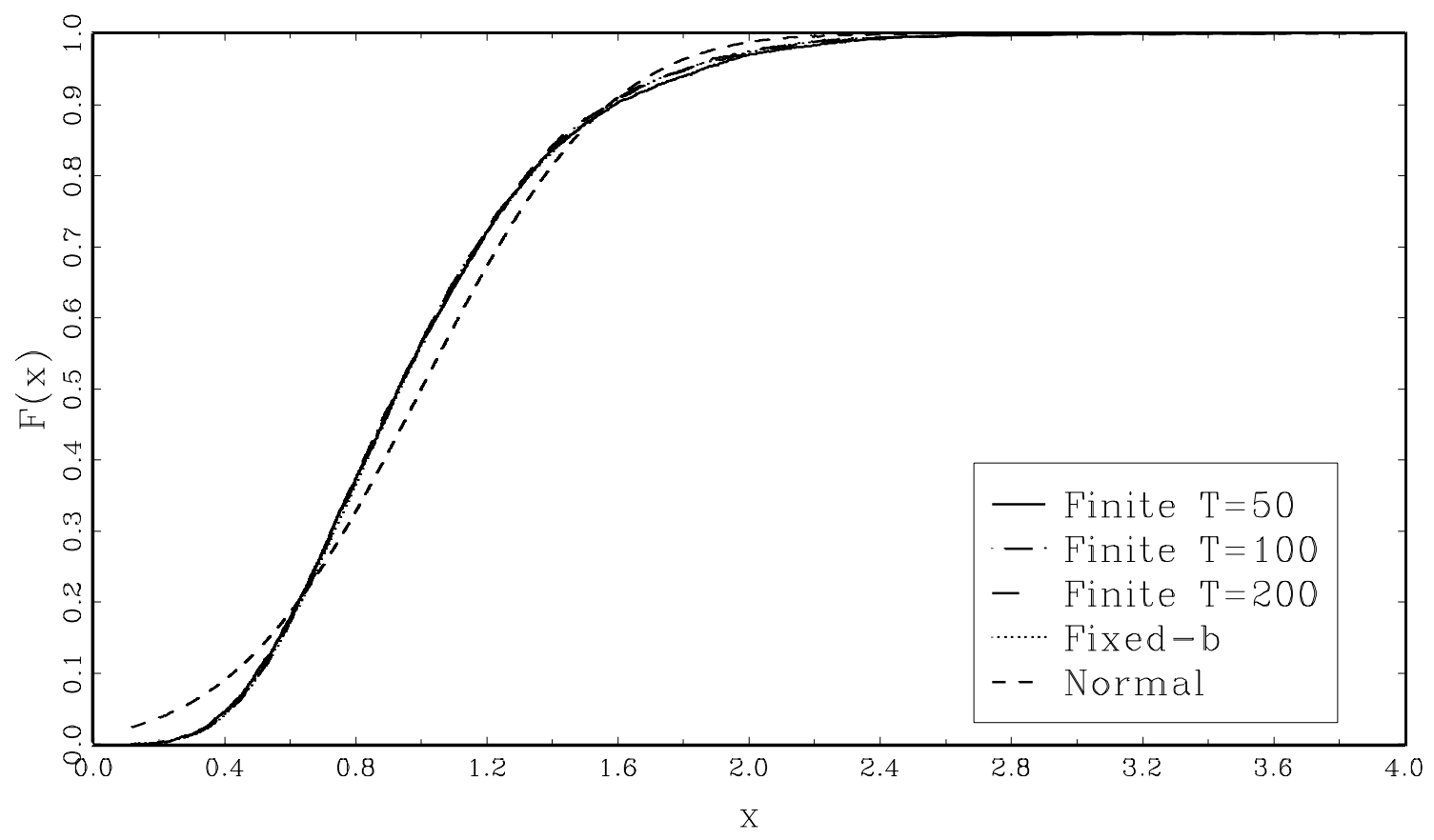

Figure 4.11: CDF Functions, QS Kernel, $\omega=0.5 \pi, \rho=0.0, \quad b=0.2$

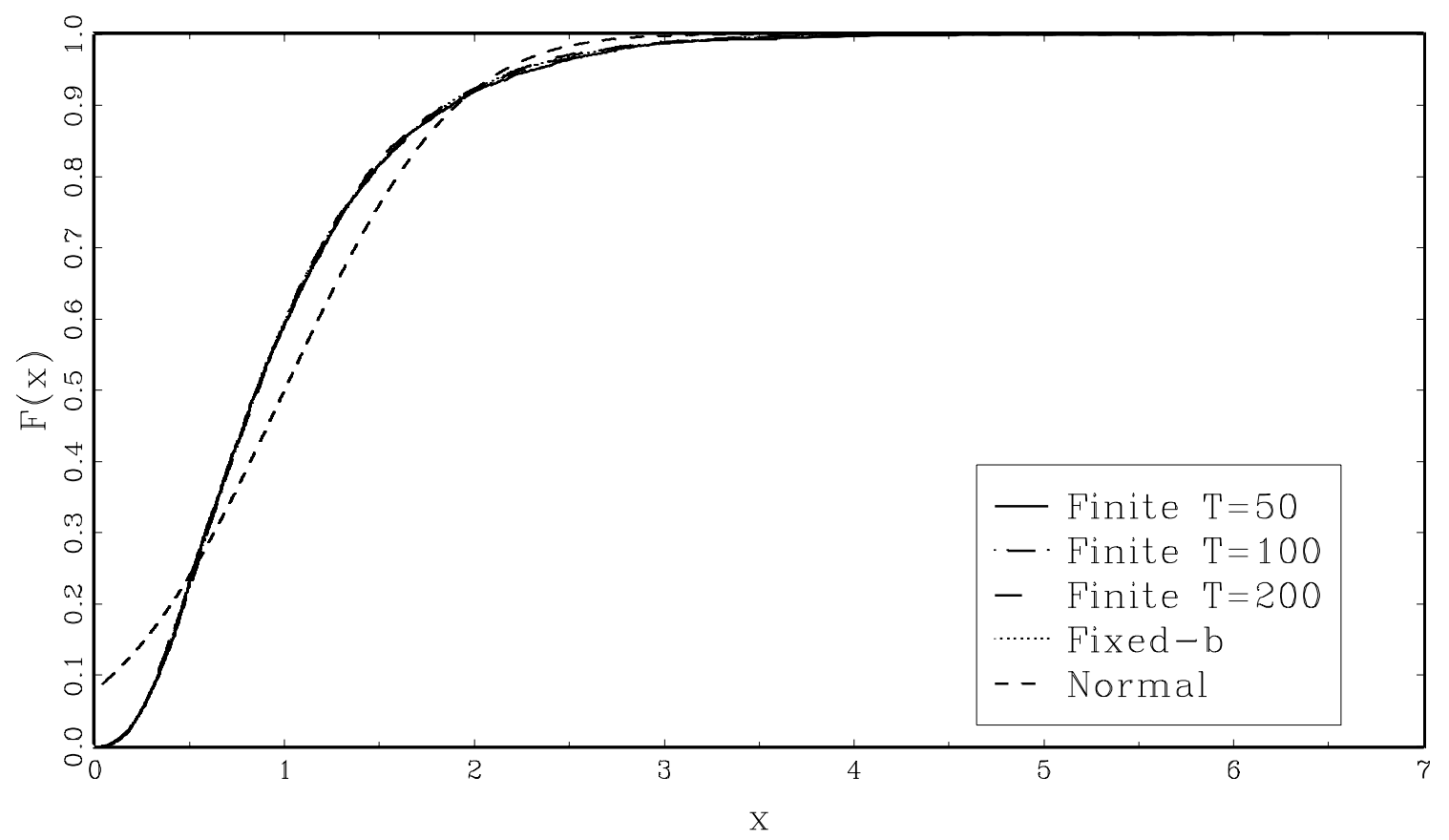

Figure 4.12: CDF Functions, QS Kernel, $\omega=0.5 \pi, \rho=0.0, b=0.5$ 


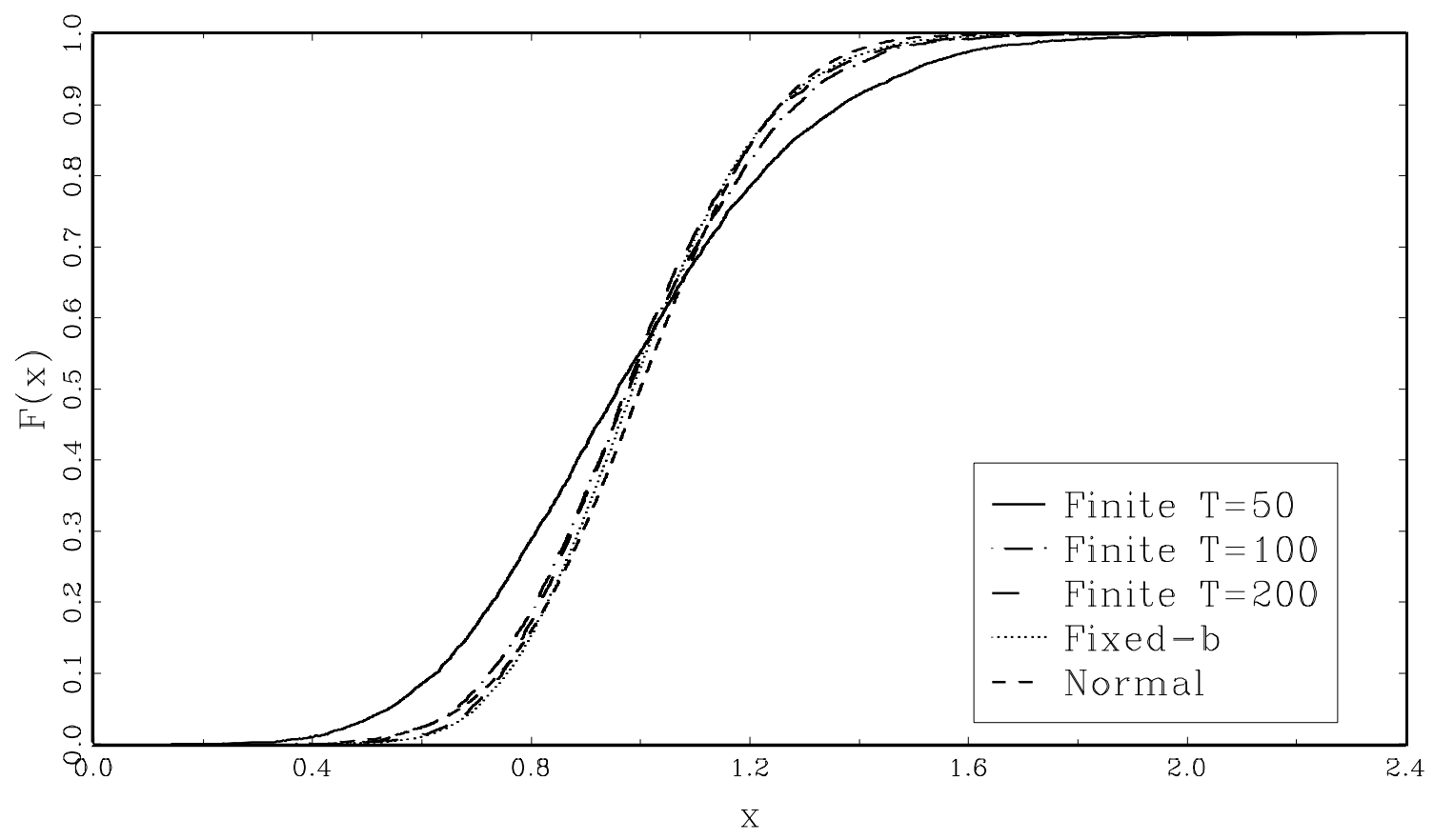

Figure 4.13: CDF Functions, QS Kernel, $\omega=0.5 \pi, \rho=-0.4, b=0.04$

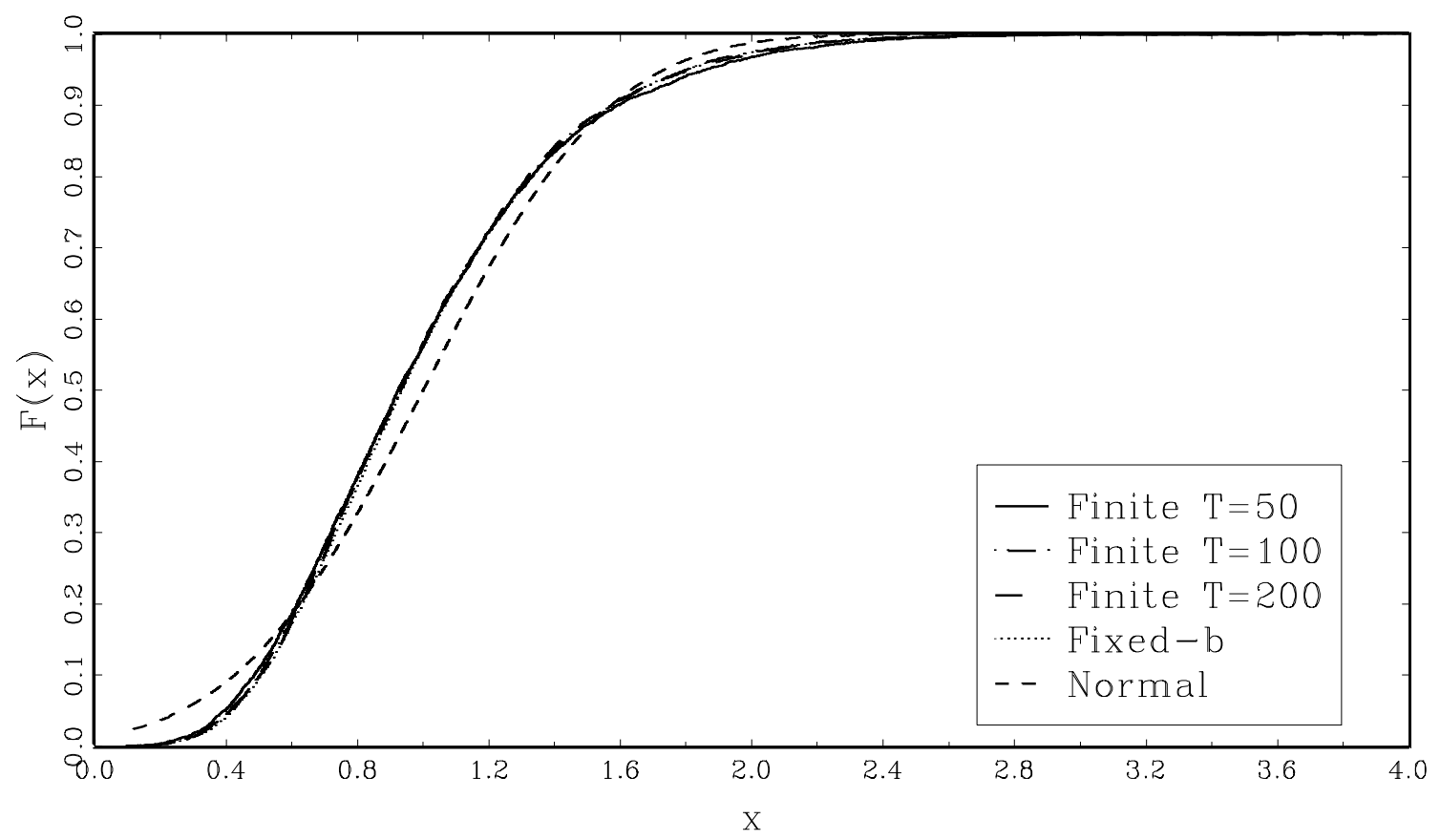

Figure 4.14: CDF Functions, QS Kernel, $\omega=0.5 \pi, \quad \rho=-0.4, \quad b=0.2$ 


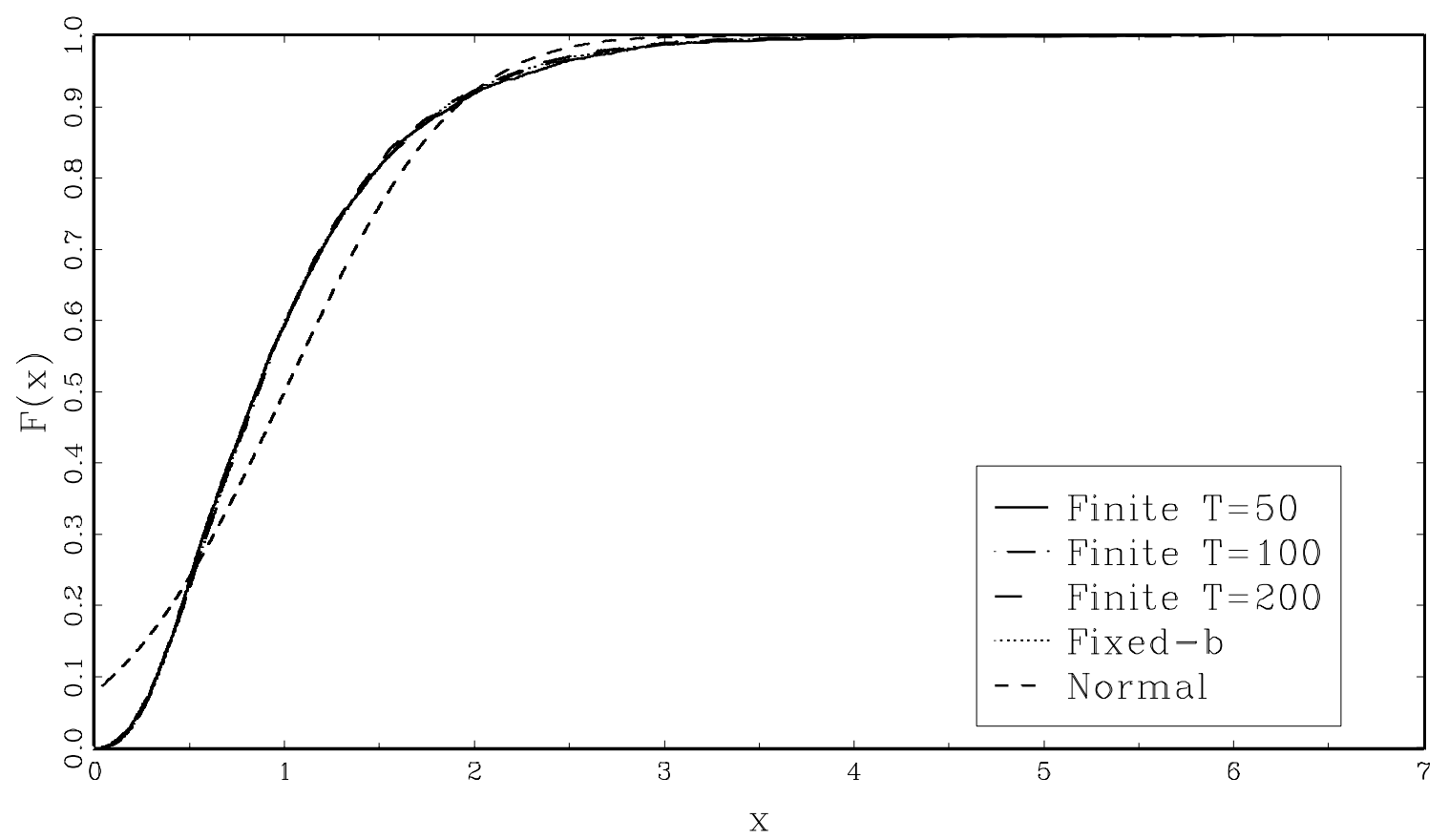

Figure 4.15: CDF Functions, QS Kernel, $\omega=0.5 \pi, p=-0.4, b=0.5$

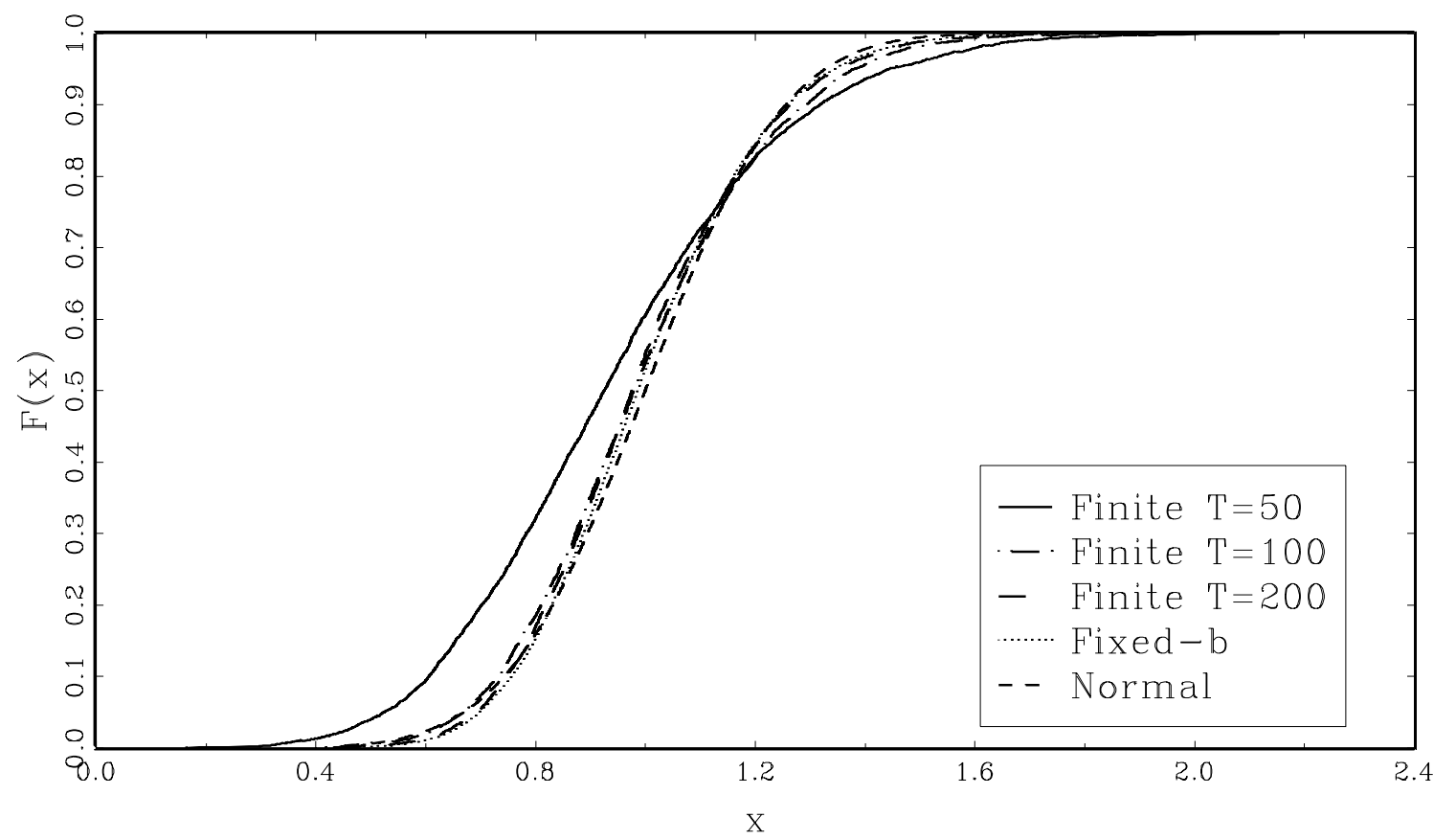

Figure 4.16: CDF Functions, QS Kernel, $\omega=0.5 \pi, \rho=0.4, \quad b=0.04$ 


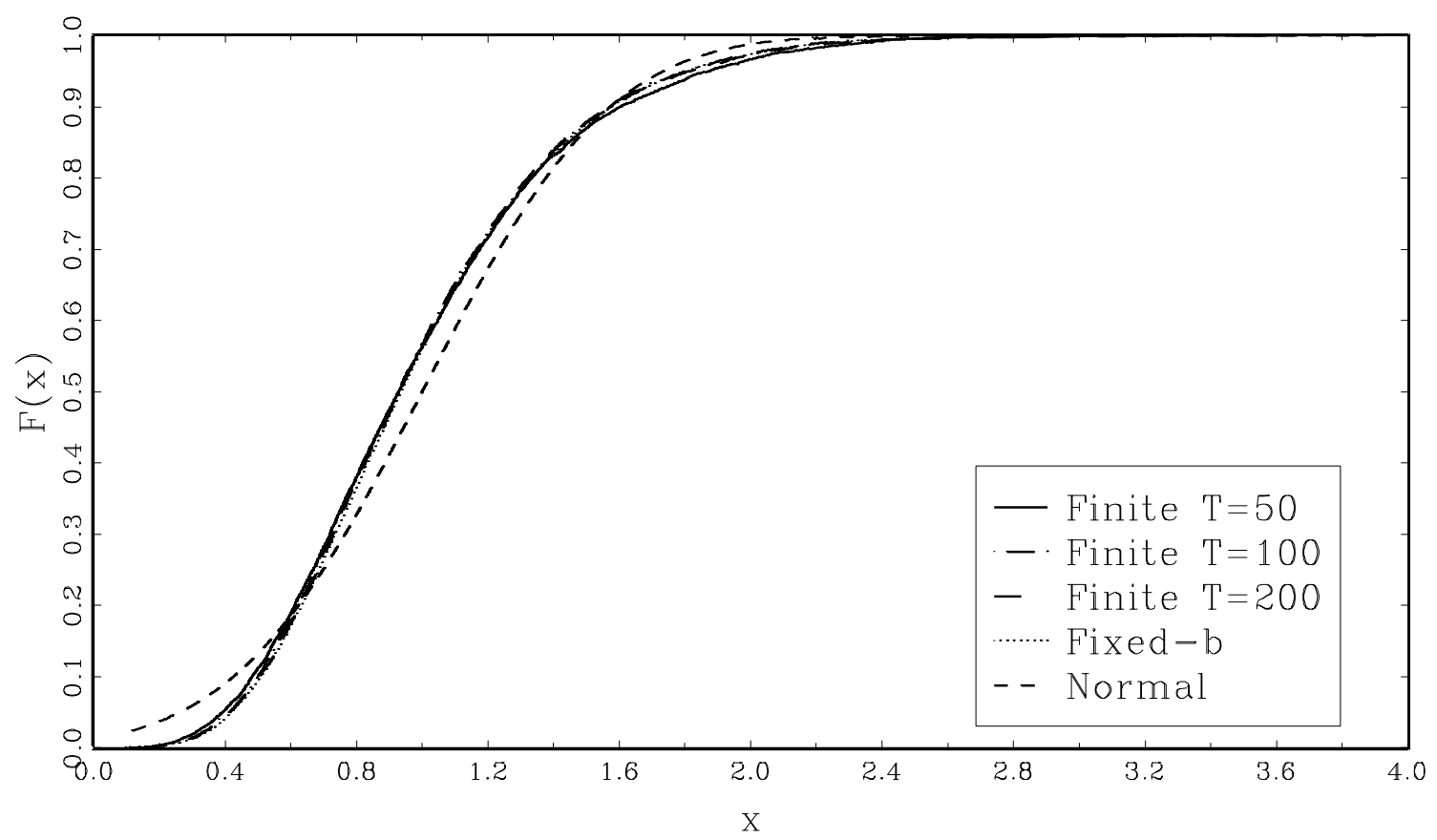

Figure 4.17: CDF Functions, QS Kernel, $\omega=0.5 \pi, \rho=-0.4, b=0.2$

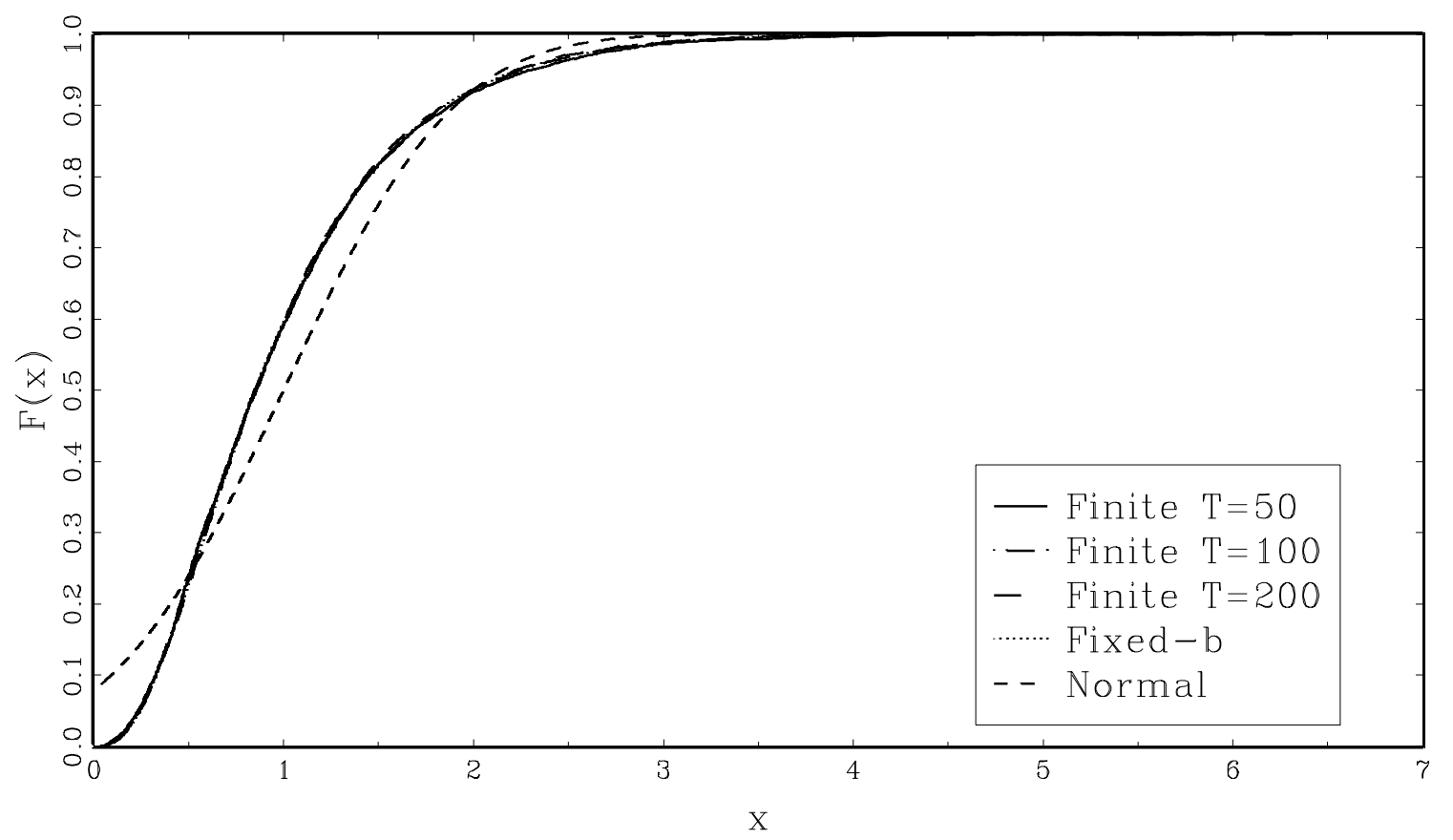

Figure 4.18: CDF Functions, Q.S Kernel, $\omega=0.5 \pi, \rho=0.4, b=0.5$ 